\title{
Background Rejection in Two-Phase Xenon using Scintillation Pulse-Shape Discrimination for Neutrinoless Double-Beta Decay Searches
}

by

\section{Braeden Thomas Veenstra}

A thesis submitted to the Faculty of Graduate and Postdoctoral Affairs in partial fulfilment of the requirements for the degree of

\author{
Master of Science \\ in \\ Physics \\ Specialization in Particle Physics \\ Ottawa Carleton Institute for Physics
}
Department of Physics
Carleton University
Ottawa, Ontario
January 2020
(C) 2020

Braeden Thomas Veenstra 


\section{Abstract}

This work attempted to identify measurable differences in the pulse shapes of one and two electron scintillation events in liquid xenon, with the goal of developing a technique for improved background rejection in neutrinoless double-beta decay $(0 \nu \beta \beta)$ experiments. A system was constructed to generate one and two electron events using gamma rays from an external thorium-228 source. Sodium iodide counters were used to tag one and two electron events, which were produced from Compton scattering and pair-production of the thorium gamma respectively. A novel cryogenic compatible silicon photomultiplier was used to measure the light from scintillation events and it was demonstrated that the system could trigger on coincidence between the scintillation and event tag signals. No conclusions could be made on the timing structure of events, based on long decay times in the photomultiplier and electronics, and suggestions are made for future improvements. 


\section{Acknowledgements}

First of all, I wish to express my gratitude towards my supervisor, Dr. David Sinclair, for his guidance and insight into both this project and the wider world of experimental physics. I would also like to thank my colleague Callan Jessiman, who I have worked with closely throughout this project.

I would further like to give my thanks to all of the faculty and graduate students who have contributed to this project or shared their advice, including Dr. Ibtessam Badhrees, Dr. Razvan Gornea, and Dr. Thomas Koffas. In particular, I want to thank my predecessor Warren Cree and Dr. Bjoern Lehnert, who worked closely with our group before moving on to UC Berkeley. Equally importantly, I would like to thank the technical staff who have assisted with the project and would especially like to thank Jeffrey Mason, James Botte, Phillippe Gravelle and Matt Bowcock, without whom none of this would have been possible.

I want to give a heartfelt thank you to all of the friends and family who have kept me motivated over the past two years. Especially I want to thank my friends Collin, Jordan and Gabriel, and my brothers Danyon and Noah. To my parents, I want to extend my deepest thanks for all of the support you have given me over the years as I pursue my education, and I particularly want to thank my mum for her tireless support. I lastly, want to give a special thanks to my dogs Maple and Taffy who have provided particular emotional support over the course of my degree.

Finally on a more informal note, I want to thank those who have indirectly helped me get this degree finished. In particular these include the wonderful staff at Rooster's Coffee House and Mike's Place Pub on campus. Without them, this thesis certainly would have never gotten finished. I would like to extend the same thanks to Beyond the Pale, Dominion City, and to the Cheshire Cat Pub, for a special kind of moral support. 


\section{Table of Contents}

Abstract ......................... ii

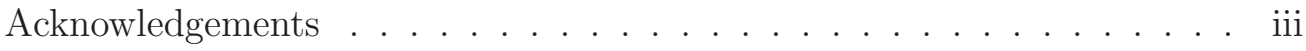

Table of Contents . . . . . . . . . . . . . . . . iv

List of Acronyms . . . . . . . . . . . . . . . vi

List of Tables . . . . . . . . . . . . . . . . viii

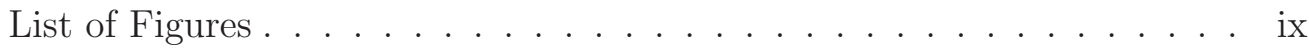

1 Introduction 1

1.1 Motivation . . . . . . . . . . . . . . . . . 1

1.2 Outline of this Thesis . . . . . . . . . . . . . . . 2

1.3 Author's Contributions . . . . . . . . . . . . . . . 3

2 Nuclear Decay and Neutrinos 5

2.1 Nuclear Decay . . . . . . . . . . . . . . . . . . . . . . . 5

2.2 Neutrinos . . . . . . . . . . . . . . . . . . . 9

2.2 .1 Leptons . . . . . . . . . . . . . . . . . . . . . 9

2.2 .2 Neutrino Properties in Standard Model . . . . . . . . . . 10

2.3 Neutrino Masses . . . . . . . . . . . . . . . . . . . . . . . . . . . 14

2.3 .1 Majorana Mass Term . . . . . . . . . . . . . . . . . . . 14

2.3.2 Neutrinoless Double Beta Decay . . . . . . . . . . . . . . 17

2.3.3 Neutrino Mass Hierarchy and Matter Effect in Neutrino Oscillations . . . . . . . . . . . . . . . . 21

3 Experimental Direction $\quad 24$

3.1 A Brief History of Double-Beta Decay . . . . . . . . . . . . . . 24

3.2 Review of Double-Beta Searches . . . . . . . . . . . . . 28

$3.2 .1 \quad$ EXO-200 and nEXO . . . . . . . . . . . . . . . . 28

3.2 .2 KamLAND-Zen . . . . . . . . . . . . . . . . . . . 30

3.2 .3 CUORE . . . . . . . . . . . . . . . . . . 31

3.2 .4 Germanium Experiments . . . . . . . . . . . . . . . 31 
4 Scintillation in Noble Gases 33

4.1 Simple Processes in Signal Generation . . . . . . . . . . . . . . 33

4.2 Timing Structure of Scintillation Events . . . . . . . . . . . 36

4.3 Two Phase Detectors . . . . . . . . . . . . . . . . . . . 40

5 Experimental Setup $\quad 42$

5.1 System Overview . . . . . . . . . . . . . . . . . . . . . . . . . . 42

5.2 Temperature and Pressure Controls . . . . . . . . . . . . . . . 45

5.3 High Voltage Control and Procedures . . . . . . . . . . . . . . . . . 49

5.4 Simulating one and Two Electron Events . . . . . . . . . . . . . 51

5.5 Light Readout . . . . . . . . . . . . . . . . . . . . . . 53

6 Analysis $\quad 56$

6.1 Sodium Iodide Detector Calibration . . . . . . . . . . . . . 56

6.2 Selection of Compton Scattered Events . . . . . . . . . . . . . . . 59

6.3 Selection of Pair-Produced Events . . . . . . . . . . . . . . . . 60

6.4 Shaping of Scintillation Traces . . . . . . . . . . . . . . . . . 62

6.5 Pulse-Shape Discrimination in Liquid Xenon Scintillation . . . . . . 64

7 Conclusions and Future Work $\quad 73$

$\begin{array}{ll}\text { References } & 76\end{array}$ 


\section{List of Acronyms}

$\mathbf{0} \nu \beta \beta$ neutrinoless double-beta decay. ix, 1, 2, 16-22, 24-27, 29, 30, 33

$2 \nu \beta \beta$ two-neutrino double-beta decay. 1, 2, 7, 17, 24, 27-29, 40

AVC active vibration control. xi, 46, 47

BEH Brout-Englert-Higgs. 13

CKM Cabibbo-Kobayashi-Maskawa. 15

CP charge conjugation and parity. 13, 15, 21

CPV CP violating. 15, 20

DAQ data acquisition. xiii, 52, 53, 55, 62

EXO Enriched Xenon Observatory. 24, 27, 29

FPSE free-piston Stirling engine. 45, 46

GUT Grand Unified theories. 17

HPGe high-purity germanium detector. 31

LAAPD large area avalanche photo-diode. 30

MPPC multi-pixel photon counter. 53

NME nuclear matrix element. 20, 26

PID proportional integral differential. 3, 46, 48

PMNS Pontecorvo-Maki-Nakagawa-Sakata. 13, 15, 20, 26 
RTD resistor temperature detector. 45, 46, 48

SiPM silicon photomultiplier. x-xii, 30, 36, 41-44, 51-55, 60, 62, 65, 66, 71, 74

SMA SubMiniature version A. 44, 45

SPDT single pole double throw. 49

SSR solid state relay. 48

TPC time projection chamber. xi, 2, 27-29, 40-42, 44, 48, 51, 52, 60, 61, 63-65, 67,74

WIPP Waste Isolation Pilot Plant. 4, 28 


\section{List of Tables}

2.1 The fundamental charged leptons and their neutrinos . . . . . . . 9 


\section{List of Figures}

2.1 Feynman diagram illustrating the weak-decay of a down quark into an up quark, by emission of a virtual $W$ boson that decays into an electron and electron anti-neutrino. In this diagram time flows from the top of the page to the bottom. The diagram was drawn using the TikZ-Feynman package for LaTeX [1]. . . . . . . . . . . 7

2.2 Feynman diagram illustrating two-neutrino double beta-decay. This decay is equivalent to the simultaneous weak decay of two bound state down quarks into two final state up quarks, two electrons and two electron anti-neutrinos. This type of event can only be observed in cases were the Q-value of singular beta decay is negative as the second vertex greatly suppresses the amplitude of process. This diagram was again drawn using the TikZ-Feynman package for $\operatorname{LaTeX}[1] \ldots$. . . . . . . . . . . . . . . . . . . . .

2.3 The leading diagram for neutrinoless double-beta decay. In this diagram, an electron anti-neutrino leaves one of the weak interaction vertices via the decay of a $W^{-}$. Since all neutrinos produced in weak decays as observed thus far are left handed and all anti-neutrinos observed so far are right handed, the anti-neutrino emitted in this vertex must be of right handed helicity. The Majorana mass term converts the outgoing right handed anti-neutrino into a left-handed neutrino, which then enters the other weak vertex. Due to the mixing from the Majorana mass, the absorption of a left handed neutrino is equivalent to the emission of a right-handed anti-neutrino. This diagram was once again drawn using the TikZ-Feynman package for $\operatorname{LaTeX}[1] \ldots \ldots$. . . . . . . . . . . . . . . . . 18 
2.4 The Feynman diagram of figure 2.3 re-imagined for the case where the mass term in the Lagrangian for the neutrino field includes both Majorana and Dirac mass terms, based on the generalizations of [2]. Like in the diagram where the sole mass term was assumed to be Majorana in nature, the first weak vertex emits a right-handed electron anti-neutrino which is converted into a left handed neutrino by the insertion of a Majorana mass term. The handedness of the neutrino is then flipped again by the insertion of a Dirac mass term so that it can be absorbed in the second weak vertex, thus completing the virtual neutrino exchange. Like all of the Feynman diagrams in this thesis, this one was drawn using TikZ-Feynman [1].

4.1 Scintillation events for relativistic electrons, $\alpha$-particles and fission fragments. Despite each type of radiation having similar energy, the scintillation profile is very different. This is because of how each type of radiation distributes deposited energy among processes that populate the ${ }^{1} \Sigma_{u}^{+}$and ${ }^{3} \Sigma_{u}^{+}$states. Electrons have a long tail, suggesting the interaction is dominated by self-trapping. Fission fragments and $\alpha$-particles each suggest a mix of self-trapping and recombination heavy processes are responsible for the resulting pulse shapes. The graph also gives measurements of the singlet and triplet lifetimes in liquid Xenon as well as the ratios of these states for different types of radiation. Curiously, note that a greater proportion of the signal seems to be lost for more ionizing types of radiation. This figured was reproduced from [3] and is used with permission. (c) American Physical Society. DOI: https://doi.org/10.1103/PhysRevB.27.5279. . . . . . . . 39

5.1 A rough diagram showing a cutaway of the inner detector. Featured in this diagram are the field cage, SiPM, spout, level probe, and grounding point. The purpose of the field cage was to establish a uniform electric field throughout the drift (liquid) region. Another copper ring is grounded to provide an even more intense electric field in the electroluminescence (gas) region. The spout, which is intended to take liquid directly to the bottom of the chamber, and the capacitive device used to measure liquid level are also shown. . 43 
5.2 A side view of the inner chamber of the TPC. In the centre are the copper rings, with the white tubing that leads the xenon to the bottom of the detector. The purpose of the tubing is to limit ripples at the surface where they might distort the light signal. At the right of the image, alongside the ruler held up to give a sense of scale, is the capacitance probe. The broader copper rings that appear outside of the stack of twelve connected rings are the grids. The top copper ring is connected to ground, which is what would have generated the high electric field in the electroluminescent detector. Care had to be taken to fill to the top grid. This photo was taken by Callan Jessiman, used with permission. . . . . . . . . . . . . . . 44

5.3 A view from underneath the inner chamber. The bottom plate to which the high voltage connections are made was not connected yet allowing for a view of the SiPM. All four pixels of the 4-channel device are visible in the photo as well as the field shaping mesh. This photo was taken by Callan Jessiman, used with permission. . . 45

5.4 A rough schematic of the intended to show the installation of the cryo-cooler. Featured are the cooler, AVC unit, water-jacket, and heater, as well as parts of the system structure. . . . . . . . . . . 47

5.5 A block diagram showing the control scheme for the boil off and recirculation of xenon. The heater boils off xenon which is then recirculated using the diaphragm pump. In the recirculation route are two purifying stages. The heating/boiling is controlled by a PID unit based on feedback from a pressure gauge in the system. The cryo-cooler is operated at a constant $70 \mathrm{~W}$ power, at which it provides a lift of $40 \mathrm{~W}$. . . . . . . . . . . . . . . . . . . 49

5.6 A thorium gamma ray enters the TPC and is Compton scattered onto the forward gamma counter. The angle was chosen so that the energy of this scattered gamma is $1022 \mathrm{keV}$. Scintillation light is then picked up by the SiPM. . . . . . . . . . . . . . . . 51

5.7 Like for the Compton scattering, pair-production is generated by interactions with a $2614 \mathrm{keV}$ thorium gamma ray. In this case, the signal is based on the annihilation of the positron that is produced in this interaction. Since these annihilations produce back-to-back gammas, some will align with both $\mathrm{NaI}$ detectors. . . . . . . . . 52 
5.8 This block diagram shows the trigger system, which is explained in the rest of this section. Labels 1-4 show the four segments of the SiPM. 5 and 6 show the forward NaI detector and the rear detector respectively. 7 indicates the edge of the TPC, which is shown from a top view. 8 shows an amplifier unit. Each of the signals from the SiPM channels and the two gamma counters each go to their own channel on the amplifier units. 9 shows the digitizer. 10 shows a fan in/out unit which converts the forward detector and SiPM signals into logic pulses. Note that the SiPM channels are summed to form the logic pulse. 11 shows a timing filter. 12 shows a discriminator unit. 13 indicates a coincidence unit. Finally, the label 14 indicates that the coincidence signal is used as the trigger for the digitizer. . 54

6.1 A Gaussian distribution was fitted to a histogram of pulse areas collected from sodium-iodide crystal detectors exposed to caesium137. The mean of these pulse areas were used as calibration points in a sodium-iodide spectrum used to identify $511 \mathrm{keV}$ gamma-rays associated with pair production and $1022 \mathrm{keV}$ gammas associated with the Compton scattering of the thorium-228 $2614 \mathrm{keV}$ gamma. The error on this peak was taken to be the error on the peak position of the fit as calculated by ROOT. The peak position and its corresponding error were used in a later linear fit calibration. For the rear detector, a double Gaussian was used as the appeared to be a shoulder effect that skewed the calibration data. . . . . . . . . 57

6.2 Gaussian peaks were fitted to the sub-ranges of the background spectra for both sodium-iodide detectors. The goal of this was to obtain extra calibration points both above and below the caesium $662 \mathrm{keV}$ line by attempting to identify the $511 \mathrm{keV}$ pair production line as well as $1460 \mathrm{keV}$ potassium-40 line. As shown, the ${ }^{40} \mathrm{~K}$ line was possible for both detectors, whereas the 511 would not have been usable for the rear detector. The pair production line was not used for calibration in either detector. . . . . . . . . . . . 58 
6.3 A broad spectrum of pulse-areas collected from the forward detector. This figure deliberately excludes excessively high energy events as well as low energy noise near the threshold, which dominated the spectrum. Notice that there is a discernible peak in the stated range for Compton energies. Note as well that there is no clearly identifiable peak in the lower energy range corresponding to possible pair-production events. The cuts on pair production are explained in the next section. . . . . . . . . . . . . . . . . .660

6.4 A partial spectrum of coincidence events in the rear detector. Low energy noise is again purposefully excluded from the histogram, and dominated the spectrum as in the forward detector. As in the case of calibration a Gaussian fit was performed on the sub-range of this peak. The sub-range was chosen based on the noticeable excess of events in the region, which would be expected at the pairproduction energy as the detector would pick these events up from both the background and the source. Based on the energy range about the excess, it is clear that there was some inaccuracy with the caesium calibration in the rear detector. . . . . . . . . . . . . 61

6.5 A sample TPC event. All events were collected with the same digitizer and DAQ software and so follow a similar structure. The binning is based on the time resolution of the digitizer so that each bin has a width of $2 \mathrm{~ns}$, which was used to calculate the time constant later. This event is just a sample, and did not correspond to either a Compton scattered or pair-production photon. . . . . . . .

6.6 A sample of one and two electron events. The pulses were chosen to show for each of pair production and Compton scattering interactions one event with a large pulse, and thus a good signal to noise ratio, and a pulse where the summed pixels are very small and may present difficulties for the fitter. The plots presented are the final processed graphs with a superimposed two term exponential fit. The parameters of these fits were used later to attempt to quantify differences between pair production and Compton scattering. . . . . 66

6.7 Histograms of the fast and slow time constants measured for pair production and Compton scattered events, fitted with a Gaussian where appropriate. This was done in order to measure the shifted fast and slow time components for each type of event. . . . . . . . . 68

6.8 The measured ratios of the fast and slow weightings for both data sets. Both very clearly represent data that are not physical due to the large number of extreme and negative events. . . . . . . . . . . 69 
6.9 Graph showing the sum of the light pulses for the entire Compton data set. . . . . . . . . . . . . . . . . 70

6.10 Graph showing the sum of the light pulses for the entire pairproduction data set. . . . . . . . . . . . . . . . . 71 


\section{Chapter 1}

\section{Introduction}

\subsection{Motivation}

For certain unstable isotopes of particular atoms beta decay is energetically forbidden, but the second-order analogous process is allowed. This second order process is usually referred to as two-neutrino double-beta decay $(2 \nu \beta \beta)$ and is an exceptionally rare event for all atoms that it has been observed in. It has long been theorized that an even rarer process may exist in which no final state neutrinos are produced, referred to as neutrinoless double-beta decay $(0 \nu \beta \beta)$.

The discovery or rejection of neutrinoless double-beta decay is a major goal of the particle physics community and a number of experiments are in progress or planned with the goal of searching for it. There are three major reasons for the interest in observing neutrinoless double-beta decay. The first of these is that it is by definition a lepton number violating process. The observation of neutrinoless double-beta decay would also confirm that neutrinos are Majorana particles and establish the mass scale of the neutrino. Finally, in addition to these reasons, the discovery of Majorana neutrinos may help to explain the relative smallness of the neutrino as well the relative abundance of matter compared to anti-matter in the universe.

The discovery potential of any given experiment is related to the expected rate of neutrinoless double-beta decay, which is extremely sensitive to what is called the "effective Majorana mass". The effective mass is in turn very sensitive to the hierarchy of neutrino mass states, which can be either normal or inverted. In the case of the normal hierarchy, which is becoming the increasingly likely reality, next generation experiments are not sensitive enough to obtain evidence for neutrinoless double-beta decay.

In addition to making larger detectors, any experiment looking to probe the normal hierarchy scenario would have to greatly reduce backgrounds. Ideally, 
backgrounds could be reduced to only the two-neutrino double-beta decay. A possible way of doing this would be to develop a technique that distinguishes between one and two electron events, as the significant backgrounds for this type of experiment produce one electron and double-beta processes, by definition, produce two.

Towards this end, a system was built to generate and tag one and two electron events in a liquid xenon detector. Scintillation light from these events was detected using an array of large area silicon photomultipliers (SiPM) and the pulse shapes of each type of event were recorded. The SiPMs used were a new model designed with a mount that could be operated at temperatures at and below that of liquid xenon. These SiPMs are also UV sensitive and particularly sensitive to the 175 $\mathrm{nm}$ scintillation light from liquid xenon. This thesis presents a research project that attempted to use pulse-shape discrimination to separate one and two electron events based on their scintillation signals in liquid xenon.

\subsection{Outline of this Thesis}

The first two chapters of this thesis will cover a brief introduction and and some of the theory behind neutrinos and $0 \nu \beta \beta$. This chapter starts by providing a short outline of the thesis and a short summary of the author's contributions to the project. The second chapter dives deeper into the theory of neutrinos. This starts with a very low level review of nuclear decay, which leads into an equally brief discussion of leptons in the standard model.

The second chapter continues with a deeper discussion on neutrino masses, starting from neutrino oscillations and how this leads to the prediction that neutrinos have non-zero masses. The latter half of the chapter discusses Majorana mass terms and neutrino-less double-beta decay, finishing with a brief discussion of the neutrino mass hierarchy and how this may affect experimental limits. This naturally leads into the third chapter which covers a brief history of neutrinoless double-beta decay $(0 \nu \beta \beta)$ and major experimental milestones in this search which is followed by a very brief discussion of some of the experiments that exist to search for this phenomenon.

The fourth chapter goes into detail on scintillation in liquid xenon, focusing on the population of the singlet and triplet states that give rise to the xenon scintillation spectrum. Particular emphasis is placed on the timing characteristics of the light output. The chapter closes with a very brief description of the concept of two-phase detection. The chapter that follows discusses the experimental set up that was used for this experiment. The most important point in the fifth chapter is the discussion of how Compton scattering and pair-production interactions of 
high energy gamma rays were used to simulate one and two electron events.

The sixth chapter covers the author's analysis of the data collected with the prototype time projection chamber (TPC) developed as part of the group. This starts by discussing the calibration of the gamma counters used in the experiment. Following discussions of calibration are separate discussions on how these were used to isolate Compton scattered and Pair-produced events separately. The chapter ends with a discussion of the attempt to then apply principles of pulse shape discrimination to the two different data sets.

The final chapter states the conclusions of the thesis and discusses how experiments of this nature might proceed with research and design in the future. This discussion comes mainly out of the fact that this thesis did not in fact establish a definitive result as to whether scintillation signals in liquid xenon can be separated into one and two electron events.

\subsection{Author's Contributions}

The author developed the slow controls for the experiment. This role was broken down into three categories: the temperature and pressure controls, the high voltage controls, and control of the cooling system. All of the temperature and pressure controls were proportional integral differential (PID) based or read only. For these controllers, there were available drivers in LabVIEW, which was the software of choice for the instrumentation. LabVIEW is released by National Instruments out of Austin, Texas. Some modifications to the drivers were necessary and or convenient ,however, and were done by the author. In addition, the author did a lot of the back panel wiring as well as all of the testing for these devices.

Initially testing of the high voltage modules was done by the author's predecessor, Warren Cree, using the interface program provided by the manufacturer of the process controllers. The author ported this control method into LaBVIEW. In addition, the author also created a more elaborate interface that allowed for the high voltage ramp to be automated. The author also tested this control system and re-calibrated the devices. Unfortunately, the high voltage itself couldn't be integrated successfully into the full system given the time constraints. This was because of the interaction of the hight voltage with the system.

The cooling unit was not, at least at the time the experiment was being set up, supported in LaBVIEW. The author obtained the basic structure for communicating with the device from a representative of the company. The full LabVIEW program that controller the cooler was then developed by the author. Further details on the cooler and other aspects of the full system are in chapter five.

In addition to producing the slow controls, the author modified and finalized 
the operation procedures for the system. The author also performed all of the full system tests in argon as well as coordinated the scintillation run with xenon. Due to the much higher boiling point and the nature of achieving stable operation, running with xenon was a two person job. The author was responsible stabilizing the system PID.

In addition to the work described above in this section, the author is listed as a member of the nEXO collaboration, as well as the EXO-200 collaboration. The author spent some time in the course of this program doing shift work at the Waste Isolation Pilot Plant (WIPP) in Carlsbad, New Mexico. This was done as part of the EXO-200 experiment. Some of these duties involved inserting and retracting the calibration source, as well as starting runs remotely. 


\section{Chapter 2}

\section{Nuclear Decay and Neutrinos}

\subsection{Nuclear Decay}

Nuclear decays are processes by which an atomic nucleus spontaneously changes to another nucleus. The most common processes are alpha decay, beta decay and gamma decay. While there are other decays - such as neutron emission, electron capture, fission, et cetera - this section will primarily review the basic three.

Alpha decay is best described as the spontaneous emission of a helium- 4 nucleus (alpha particle) from a much heavier atom. This is most commonly observed in transuranic elements which have a very large number of protons and neutrons. In equation form, this is described as in equation 2.1 .

$$
{ }_{Z}^{N} A \longrightarrow{ }_{Z-2}^{N-4} A^{*}+{ }_{2}^{4} H e^{2+}
$$

The symbol $A$ has been used as a stand in to represented any atomic nucleus, $N$ is the atomic mass, and $Z$ is the proton number. The alpha particle released in this process is typically in the $\mathrm{MeV}$ range. Alpha particles have short range in most materials because of their relatively high mass and +2 charge.

On the right hand side of equation 2.1, I have used a star to denote that the daughter nucleus is in an excited state. Excited state nuclei are the common source of gamma emissions. The nucleus de-excites, releasing a high energy photon in the process. This typical process is illustrated in equation 2.2.

$$
{ }_{Z}^{N} A^{*} \longrightarrow{ }_{Z}^{N} A+\gamma
$$

Here, the symbol $\gamma$ represents a photon. It is also possible to have the atom de-excite through multiple levels. In fact, this is far more common in which case the process is multi-stage. Decays with multiple intermediate levels produce a spectrum of gamma energies. 
Finally, we have beta decay. Beta decay can take two forms. The first, usually called beta negative or just beta decay, occurs when a neutron decays into a proton releasing an electron and electron anti-neutrino as illustrated in equation 2.3 below.

$$
{ }_{Z}^{N} A \longrightarrow{ }_{Z+1}^{N} A^{*}+e^{-}+\overline{\nu_{e}}
$$

The other form, beta positive decay is the inverse process. In this decay scheme, a proton is converted to a neutron, releasing a positron (anti-electron) and an electron neutrino. This is illustrated in equation 2.4 below.

$$
{ }_{Z}^{N} A \longrightarrow{ }_{Z-1}^{N} A^{*}+e^{+}+\nu_{e}
$$

Typically, when the literature speaks of beta decay, the negative case is what is meant. Beta-positive decay is more ordinarily referred to as positron emission, but is included since it follows the same mechanism. Unlike alpha decay, which is a nuclear process, and gamma radiation, which is produced in the de-excitation of energized nuclei, Beta decay occurs at the level of fundamental particle physics. The neutron that decays is a composed of an up quark and two down quarks. The proton is similarly a composite of two up quarks and a down quark. In particle physics terms, one of the bound-state down quarks that contributes to the neutron undergoes a weak decay by emitting a $W^{-}$boson. Shown below in equation 2.5.

$$
d \longrightarrow u+W^{-}
$$

The final state anti-neutrino and electron are then produced in the decay of the $W$-boson shown in equation 2.6

$$
W^{-} \longrightarrow \overline{\nu_{e}}+e^{-}
$$

In all known nuclear decays, the energy released is not enough to produce the $W^{-}$ boson as

$$
Q_{\beta}<<M_{W}
$$

in which case the down quark decays directly to the final state anti-neutrino and electron as shown in equation 2.8.

$$
d \longrightarrow u+\overline{\nu_{e}}+e^{-}
$$

The process in equation 2.8 is then said to be mediated by a virtual $W^{-}$. This is perhaps best illustrated using a Feynman diagram. 


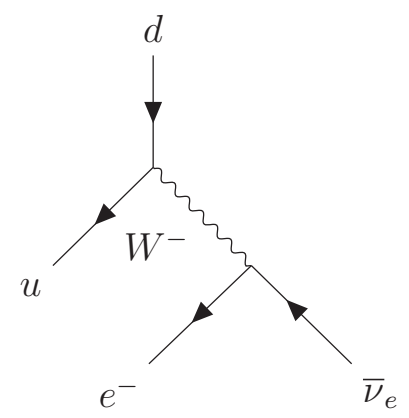

Figure 2.1: Feynman diagram illustrating the weak-decay of a down quark into an up quark, by emission of a virtual $W$ boson that decays into an electron and electron anti-neutrino. In this diagram time flows from the top of the page to the bottom. The diagram was drawn using the TikZ-Feynman package for LaTeX [1].

An important element in the detection of $\beta$ decays is the energy spectrum of the decay. Per conservation of energy the total energy of each particle in any event must add up to the Q-value for the process. Similarly, in order to satisfy conservation of momentum, the final state momenta must sum to zero ${ }^{1}$. For the decay illustrated in figure 2.1 there are three final state particles and hence a continuous spectra of final state momenta, and thus energies may satisfy the conservation laws. In the specific case of beta-decay the energy can only be reconstructed from the electron and recoiling nucleus as individual neutrinos react too weakly.

There are some circumstances where more complicated weak decays can occur. To understand these physicists use the concept of the Q-value. By definition the Q-value is the total energy released in a decay. In other words any nuclear process with a negative Q-value implies a final state in which energy is consumed and thus is forbidden. Isotopes that don't undergo beta decay are typically prevented from the decay by a negative Q-value between the daughter isotope as illustrated in equation 2.9 .

$$
Q\left({ }_{Z}^{N} A \longrightarrow{ }_{Z+1}^{N} A^{*}+e^{-}+\overline{\nu_{e}}\right)<0
$$

There are some isotopes, however, for which beta-negative decay has a negative Q-value but a process equivalent to two simultaneous beta-negative decays has a Q-value that is positive. This condition is shown in equation 2.10.

$$
Q\left({ }_{Z}^{N} A \longrightarrow{ }_{Z+2}^{N} A^{*}+2 e^{-}+2 \overline{\nu_{e}}\right)>0
$$

This situation is referred to as two-neutrino double-beta decay $(2 \nu \beta \beta)$, and has

\footnotetext{
${ }^{1}$ Must sum to the momentum of the decaying quark, which is assumed to be reasonably close to zero compared to the relativistic momenta of the decay.
} 
been observed in several isotopes. Cast in the form of a weak decay this is more appropriately described as the simultaneous decay of two down quarks into a pair of up quarks, anti-neutrinos and electrons as shown in equation 2.11.

$$
d d \longrightarrow u u+2 e^{-}+2 \overline{\nu_{e}}
$$

There are isotopes for which the $\mathrm{Q}$ value is positive for both standard beta decay, as well as double beta decay. In all practical terms however, it is impossible to measure the latter decay due to the fact that the decay probability is greatly reduced by the second vertex so that

$$
t_{1 / 2}\left(Q_{\beta}>0\right)<<t_{1 / 2}\left(Q_{\beta \beta}>0\right)
$$

making it much more likely that the isotope decays by singular beta decay before the other can be observed. Attractive candidates to measure the properties of double-beta decay then must satisfy both equation 2.9 and equation 2.10. Like for the ordinary beta decay, double beta decay can be easily illustrated using a Feynman diagram. The dominant diagram is shown in figure 2.2.

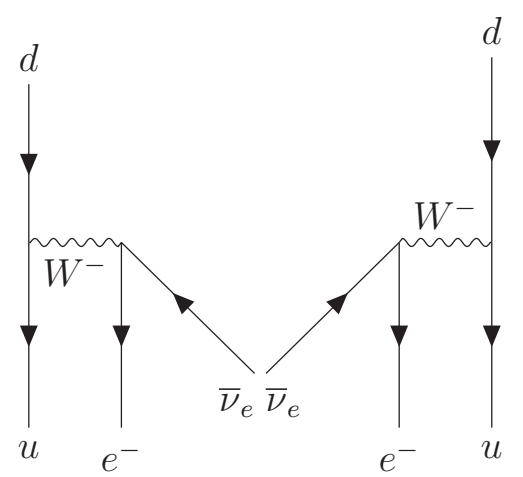

Figure 2.2: Feynman diagram illustrating two-neutrino double beta-decay. This decay is equivalent to the simultaneous weak decay of two bound state down quarks into two final state up quarks, two electrons and two electron anti-neutrinos. This type of event can only be observed in cases were the Q-value of singular beta decay is negative as the second vertex greatly suppresses the amplitude of process. This diagram was again drawn using the TikZ-Feynman package for LaTeX [1].

As outlined in the introduction to this thesis there are predictions of a possible neutrino-less mode by which the process illustrated in 2.2 may progress. As the name suggests, these modes contain no final state neutrinos or anti-neutrinos. The theoretical possibility of neutrino-less double-beta decay is motivated by the fact that neutrinos have mass and that this mass may derive from the so-called 'Majorana mass term'. The remainder of this chapter will be dedicated to explaining 
how massive Majorana neutrinos lead to the prediction of neutrino-less double beta decay. In addition, it will also explain why the half lives of neutrino-less events are so small and why they may in fact be even smaller than previously thought.

\section{$2.2 \quad$ Neutrinos}

\subsubsection{Leptons}

The properties of the neutrino will set up discussions on neutrino-less double beta decay later in this chapter. Simply stated, neutrinos are fundamental particles in the standard model. They belong to a class of fundamental particles called leptons. A lepton is defined as a colourless, spin 1/2 fermion, and comes in three flavours or generations: electron, muon, and tau. Each generation has a neutrino and a particle carrying elementary charge named according to the generation. These particles are summarized in table 2.1. Neutrinos were originally predicted to be massless, however it would turn out that this is not the case. Since leptons are fermions, they have corresponding anti-particles.

\begin{tabular}{|c|c|c|}
\hline Generation & Charged & Neutrino \\
\hline 1 & $\mathrm{e}^{-}$ & $\nu_{e}$ \\
\hline 2 & $\mu^{-}$ & $\nu_{\mu}$ \\
\hline 3 & $\tau^{-}$ & $\nu_{\tau}$ \\
\hline
\end{tabular}

Table 2.1: The fundamental charged leptons and their neutrinos

In all observed decays, the conservation of lepton number is an observed symmetry. What this means is that if an interaction has a number, $n$, of leptons coming into the vertex of the interaction, then the product of the interaction must also contain that same number of leptons. Or at the very least, this rule is observed to hold in all known interactions. There is not a strict theoretical reason as to why this should hold true. Lepton number conservation is better illustrated using an example, the decay of atmospheric muons. These decays are illustrated in equation 2.13 and equation 2.14 .

$$
\begin{aligned}
& \mu^{-} \longrightarrow e^{-}+\overline{\nu_{e}}+\nu_{\mu} \\
& \mu^{+} \longrightarrow e^{+}+\nu_{e}+\overline{\nu_{\mu}}
\end{aligned}
$$

Equation 2.13 shows the decay of a muon into an electron and two neutrinos, and equation 2.14 shows the decay of the muon's antiparticle - usually referred to as a mu positive. The left handed side of equation 2.13 has a single muon, which is 
said to have a lepton number of 1 . Also of importance is the fact that the lepton is a muon, as flavour is conserved as well. Moving to the right hand side of equation 2.13, there are three products: an electron, an electron anti-neutrino, and a muon neutrino. The electron is assigned electron number +1 while the electron antineutrino is assigned electron number -1 , leaving just the muon number of +1 due to the muon neutrino. This is what is meant by lepton number conservation. The total number of leptons is conserved with flavour. Equation 2.14 is the same as equation 2.13 with all particles converted to their anti-particles.

\subsubsection{Neutrino Properties in Standard Model}

The previous section looked at the very basic characteristics of leptons as a whole. This section will cover some properties of the neutrino specifically. Neutrinos carry neither electrical charge nor colour. This means that neutrinos do not interact with either the electromagnetic force or the strong force. In other words, all interactions with neutrinos are mediated by the weak force. This is problematic for detection since the weak processes are suppressed by the masses of the weak carriers, the $W_{-}^{+}$ and $Z_{0}$ bosons $^{2}$. This combines with the fact that neutrino masses are so light that any reasonable interaction produces them at ultra-relativistic energies, where their properties are more difficult to measure [6]. A common phrase in presentations on neutrino physics is that a single neutrino can pass through a light-year of lead before it has any significant chance of interaction. They were only successfully discovered due to the large flux from solar and atmospheric sources. Even with huge numbers, this feat required very large, very sensitive detectors.

There are two types of weak interaction, charged and chargeless. Chargeless weak interactions are mediated by the $Z_{0}$ particle and include all weak scattering events. Flavour changing, or charged, weak interactions are mediated by the $W_{-}^{+}$ bosons. An example of charged weak decays would be the interactions shown in equations 2.13 and 2.14 that were discussed in the previous section. Beta decay is also an example of a charged weak interaction. Protons and neutrons are composite states with the neutron being represented by an up quark and two down quarks (udd) and the proton by two up quarks and a down quark (uud). The weak decay of the proton to a neutron actually occurs by the spontaneous emission of a virtual $W^{-}$boson by one of the bound state up quarks that make up the proton, illustrated as

$$
u \longrightarrow W^{-}+d
$$

\footnotetext{
${ }^{2}$ Discussed in lectures by Prof. Bruce Campbell and Prof. Daniel Stolarski. These are based on effective field theories and these arguments can be derived from material on weak interactions in [4] and effective field theories in [5].
} 
The virtual $W^{-}$then decays into an electron and anti-neutrino.

Another prediction of the standard model is the expectation of massless neutrinos. Neutrinos were originally expected to be massless due to the lack of any operator returning mass to neutrino fields. Recent discoveries, however, have confirmed that neutrinos are massive particles - though this mass is very small. It was already discussed in the previous chapter that SNOLab [7] and SuperKamiokande [8] have established that neutrino states oscillate based on measurements of solar and atmospheric neutrinos respectively. An oscillation means that neutrinos change flavour states. In other words, using the SNO example, one can calculate the expected number of electron neutrinos produced on a per unit time basis in the sun. Based on this, one acquires an expected flux of solar neutrinos that can be measured in a detector on earth. In the case of SNO, this was a Water-Cerenkov detector. Based on SNO's measurements, the number of neutrinos actually measured is smaller than what one would calculate from the expected flux. The SNO collaboration was able to show that this was due to the fact that some of the electron neutrinos changed flavour to muon and tau neutrinos en-route from their point of origin. SuperKamiokande showed a similar result for atmospheric neutrinos, and representatives for both collaborations were awarded the Nobel prize in physics in 2015.

One consequence of the discovery that neutrinos oscillate is to demonstrate that the standard model assumption of massless neutrinos is incorrect. Neutrino oscillations are a topic which come up in elementary particle physics courses at the graduate level. The discussion here will follow that found in David Griffiths' Introduction to Elementary Particles [4] with some use of the formalism of Fantini et al [9]. The basis for neutrino oscillations is the idea that the neutrino flavour states that have been detected and measured $^{3}$, are not the true eigenstates of the neutrino-field Hamiltonian. The true eigenstates in this formulation are linear combinations of the flavour states. To see why this implies neutrino masses, let's consider the case in which oscillations occur only between the first two flavour states. In this case we have the mixing matrix ${ }^{4}$

$$
\left(\begin{array}{l}
\nu_{1} \\
\nu_{2} \\
\nu_{3}
\end{array}\right)=\left(\begin{array}{ccc}
\cos \theta_{12} & \sin \theta_{12} & 0 \\
-\sin \theta_{12} & \cos \theta_{12} & 0 \\
0 & 0 & 1
\end{array}\right)\left(\begin{array}{l}
\nu_{e} \\
\nu_{\mu} \\
\nu_{\tau}
\end{array}\right)
$$

It is easy to verify that the normalization condition is satisfied for both of these

\footnotetext{
${ }^{3}$ See table 2.1

${ }^{4}$ The author has defined each of the stationary states opposite Griffiths treatment. The purpose of this is to keep consistent with notation used in Fantini et al. The reasons for this will become apparent shortly.
} 
states. This matrix gives us the stationary states as

$$
\begin{array}{r}
\left|\nu_{1}\right\rangle=\cos \theta_{12}\left|\nu_{e}\right\rangle+\sin \theta_{12}\left|\nu_{\mu}\right\rangle \\
\left|\nu_{2}\right\rangle=-\sin \theta_{12}\left|\nu_{e}\right\rangle+\cos \theta_{12}\left|\nu_{\mu}\right\rangle .
\end{array}
$$

For any quantum state, the time dependence can be easily returned.

$$
|\psi(t)\rangle=e^{-E t}|\psi\rangle
$$

Where in equation 2.18 the convention

$$
\hbar=c=e=1
$$

has been used. Griffiths follows by making the assumption that at time $t=0$, the state is an electron neutrino such that

$$
\begin{array}{r}
\left|\nu_{1}(0)\right\rangle=\cos \theta_{12} \\
\left|\nu_{2}(0)\right\rangle=-\sin \theta_{12} .
\end{array}
$$

Using the equations for the stationary states from 2.17 , the equation for a muon neutrino at some time, $t$, later can be written

$$
\left|\nu_{\mu}\right\rangle=\sin \theta_{12}\left|\nu_{1}\right\rangle+\cos \theta_{12}\left|\nu_{2}\right\rangle
$$

which simplifies to

$$
\left|\nu_{\mu}\right\rangle=\sin \theta_{12} \cos \theta_{12}\left(e^{-E_{1} t}-e^{-i E_{2} t}\right) .
$$

Despite the reversals of the definitions of the stations of the stationary states, the algebra from this point is essentially identical to that found in Introduction to Elementary Particles [4], which produces the result

$$
P_{\nu_{m u} \rightarrow \nu_{e}}=\langle\nu \mid \nu\rangle=\left[\sin 2 \theta_{12} \sin \left(\frac{E_{2}-E_{1}}{2}\right)\right]^{2} .
$$

From the result in equation 2.20, it is clear that the oscillation of neutrino states requires that neutrinos have mass. Consider from special relativity that

$$
E^{2}=p^{2}+m^{2}
$$


where $E$ is the energy of the particle, $p$ is its momentum and $m$ is its rest mass. It is clear that in an oscillation scenario, total energy must be conserved. In the case of massless neutrinos, $E=p$, so any energy difference must be zero and hence oscillation cannot occur as the probability in equation 2.20 likewise goes to zero. Griffiths goes further and derives the dependence on the mass splitting to give

$$
P_{\nu_{m u} \rightarrow \nu_{e}}=\langle\nu \mid \nu\rangle=\left[\sin \left(2 \theta_{12}\right) \sin \left(\frac{\Delta m_{21}}{2 E}\right)\right]^{2}
$$

The complete mixing matrix is known as the Pontecorvo-Maki-Nakagawa-Sakata (PMNS) matrix.

$$
U=\left(\begin{array}{ccc}
c_{12} c_{13} & s_{12} c_{13} & s_{13} e^{-i \delta_{C P}} \\
-s_{12} c_{23}-c_{12} s_{23} s_{13} e^{i \delta_{C P}} & c_{12} c_{23}-s_{12} s_{23} s_{13} e^{i \delta_{C P}} & s_{23} c_{13} \\
s_{12} s_{23}-c_{12} c_{23} s_{13} e^{i \delta_{C P}} & -c_{12} s_{23}-s_{12} c_{23} s_{13} e^{i \delta_{C P}} & c_{23} c_{13}
\end{array}\right)
$$

Fantini et al [9] parametrizes $U$ in terms of four matrices. If the charge conjugation and parity $(\mathrm{CP})$ violation is ignored, then $U$ may be written as

$$
U=U_{23} U_{13} U_{12}
$$

Which when expanded is:

$$
U=\left(\begin{array}{ccc}
1 & 0 & 0 \\
0 & \cos \theta_{23} & \sin \theta_{23} \\
0 & -\sin \theta_{23} & \cos \theta_{23}
\end{array}\right)\left(\begin{array}{ccc}
\cos \theta_{13} & 0 & \sin \theta_{13} \\
0 & 1 & 0 \\
-\sin \theta_{13} & 0 & \cos \theta_{13}
\end{array}\right)\left(\begin{array}{ccc}
\cos \theta_{12} & \sin \theta_{12} & 0 \\
-\sin \theta_{12} & \cos \theta_{12} & 0 \\
0 & 0 & 1
\end{array}\right)
$$

Notice that the matrix $U_{12}$ is simply the mixing matrix from equation 2.16. When written in this way, it becomes clear that neutrino mixing is dependent on mass splittings $\Delta m_{12}, \Delta m_{23}$ and $\Delta m_{13}$, at least two of which must be independent. Unfortunately, measuring mass splitting only gives the magnitude of the differences of the neutrino masses and very little insight into the values of the neutrino mass eigenstates themselves. The mass squared splittings are quantities that have been measured. The values of these measurements are reported in [10] as $\Delta m_{21}^{2}=7.54 \times 10^{-5} \mathrm{eV}^{2}$ and $\left|\Delta m_{31(23)}\right|^{2}=2.47(2.46) \times 10^{-3} \mathrm{eV}^{2}$.

For most massive standard model particles, mass could be returned via the Brout-Englert-Higgs $(\mathrm{BEH})^{5}$ mechanism. In the case of neutrinos, this is not a satisfying answer since constraints on the neutrino mass are so small in comparison to any other fundamental particles [11]. Bilenky 2015 cites the best limit on the

\footnotetext{
${ }^{5}$ Often referred to simply as the 'Higgs' mechanism.
} 
neutrino mass as the 2013 established by the Planck Collaboration [12],

$$
\Sigma m_{\nu}<0.23 \mathrm{eV}
$$

As of Summer 2018, the Planck collaboration claimed to have reduced this limit to

$$
\Sigma m_{\nu}<0.12 e V
$$

in an article awaiting publication in the journal Astronomy \& Astrophysics [13]. The author is unaware of a lower limit. It is worth pointing out again that, as experimental results from cosmology constrain the summed neutrino masses further, it increases the likelihood that $0 \nu \beta \beta$ experiments must probe the normal hierarchy, and thus higher than the target $10^{28}$ year sensitivity of proposed tonnescale experiments.

\subsection{Neutrino Masses}

\subsubsection{Majorana Mass Term}

This final section will discuss some of the consequences of the existence of massive neutrinos. The fact that neutrinos are neutral fermions opens up interesting possibilities concerning the properties of this mass. In particular the nature of the neutrino mass is still unknown. To elaborate on what this means, consider the Dirac equation

$$
(i \not \partial-m) \psi=0 .
$$

Equation 2.28 is the model that predicted the existence of antiparticles. The solutions to this equation are typically presented in four-vector notation and have both positive and negative energy solutions. This presented a problem since it was traditionally understood that energy couldn't be negative, especially in field theory where the low energy limit is the rest mass of the particle. Dirac thought of this in terms of vacuum sitting on top of a "sea of negative energies". When fermions are created they are formed in pairs. One can be thought of as a particle propagating in space forward through time. The other can then be thought of as a "hole" in the negative energy sea propagating backwards through time. Antiparticles are then defined as these negative energy propagations.

A consequence of defining antiparticles this way is that the sign of electrical charge flips when transforming from particle to antiparticle. All fundamental fermions whose masses have been measured so far are charged, and thus potential terms in a Lagrangian that mix particle and antiparticle are not allowed due to 
conservation of electric charge. Since neutrinos do not carry any electric charge, this leaves open the possibility that such a term may exist as an extension of the neutrino field in the standard model. The Majorana mass is such a term, and the simplest expression simply mixes particle and antiparticle $[14,2,6]$

$$
\mathcal{L}_{M}=-M_{M} A \bar{A}+h . c .
$$

Where $A$ is an arbitrary neutral fermion and $\bar{A}$ its antiparticle. Kayser $[2,6]$ extends this to the general left handed neutrino field.

$$
\mathcal{L}_{M_{\nu}}=-\frac{1}{2} M_{M} \overline{\nu_{L}^{C}} \nu_{L}+\text { h.c. }
$$

Where the superscript $C$ denotes the charge conjugate of the left handed antineutrino, in other words the right handed anti-neutrino observed in real life. Terms of the form of 2.30 effectively equate particles with their antiparticles. Terms of this form allow for interesting physical effects.

As a first example, consider again the PMNS matrix of equation 2.23. It was shown by Kobayashi and Maskawa [15] that a system of mixed states with greater than two states allow for a CP non-conserving phase. This effect was largely discussed in terms of the weak decays of hadrons and was used to explain the CP violating effects of Kaon interactions $[16]^{6}$. Cabibbo [16] proposed that the effect could be extended to the neutrino sector and would have physical consequences. In the case of Majorana neutrinos there are also oscillations that can occur along the diagonal, generating two additional CP violating phases[14 $]^{7}$ which are discussed in more detail by Petcov [10], as well as Girardi et al[17]. The Majorana CP violating $(\mathrm{CPV})$ phases are parametrized in terms of a diagonal matrix

$$
P=\left(\begin{array}{ccc}
1 & 0 & 0 \\
0 & \exp \left(\frac{i \alpha_{21}}{2}\right) & 0 \\
0 & 0 & \exp \left(\frac{i \alpha_{31}}{2}\right)
\end{array}\right)
$$

which can be added back to equation 2.23 [17] by taking

$$
V=U P
$$

where a new mixing matrix, $V$, has been defined to include the possible Majorana violating phases. The potential of discovering extra CPV phases is a major moti-

\footnotetext{
${ }^{6}$ Part of the Cabibbo-Kobayashi-Maskawa (CKM) matrix used to describe quark mixing in weak decays. This matrix takes the same form as the analogous PMNS matrix, though the measured values of the mixing angles are different

${ }^{7}$ Referenced as the origin of these phases in [17]
} 
vating factor in searching for Majorana neutrinos. The reason for this is that such $\mathrm{CP}$ violation may be able to account for the baryon asymmetry in the universe [10].

Finally, it is worth noting that a term that includes both a Dirac and a Majorana mass term may be present for the neutrino fields. In this case, Kayser [2] gives the simplest Lagrangian as:

$$
\mathcal{L}_{M_{\nu}}=-\frac{1}{2} M_{M}\left(\overline{\nu_{L}^{C}} \nu_{R}+\overline{\nu_{R}^{C}} \nu_{L}\right)+M_{D} \overline{\nu_{R}} \nu_{L}+\text { h.c.. }
$$

Of interest in equation 2.33 is the fact that it allows for neutrino anti-neutrino mixing, and can be modified to allow for the possibility of physical right-handed sterile $^{8}$. It can be shown that the stationary states of a Hamiltonian generated from equation 2.33 are Majorana in nature[2], and so any physical neutrino state will still be a Majorana particle and undergo any interesting reactions for this case. Of primary concern, a Lagrangian of the form of 2.33 still predicts a nonzero amplitude for the $0 \nu \beta \beta$ process that will be discussed in the next section. It is mainly included for completeness and to reinforce the idea that the discovery of sterile right-handed neutrinos ${ }^{9}$ does not immediately rule out the possibility of Majorana neutrinos or neutrinoless double-beta decay.

It may in fact be the case that a Lagrangian containing both Dirac and Majorana mass terms may be the more convenient and natural expression for the neutrino fields. The reason for this is that such a model can be tailored to account for the relative smallness of the neutrino masses through what is known as a see-saw mechanism. Specifically, a popular explanation is the type one see-saw mechanism ${ }^{10}$. It can be shown [18] that a Lagrangian of the form of 2.33 can be used to construct two mass states

$$
m_{-}=m_{D}^{2} / M-M
$$

and

$$
m_{+}=m_{M}\left(1+\frac{m_{D}^{2}}{m_{R}^{2}}\right) .
$$

If it is further assumed that the Dirac component of the mass is significantly smaller than the Majorana term,

$$
M_{D}<<M_{M}
$$

\footnotetext{
${ }^{8}$ So called because they do not react with standard model particles

${ }^{9}$ For example as a potential dark matter candidate.

${ }^{10}$ Though there are other possible mechanisms giving neutrinos mass based on a similar principle, for example see $[10,18]$
} 
then the Majorana component surpresses the mass of one of the neutrino states, hence the see-saw term, and explains the lightness of observed left-handed neutrino masses. It's worth noting that type one see-saw theories also predict a very heavy right-handed sterile neutrino of mass on the order of the Majorana mass term. Such a massive non-interacting particle is naturally a dark matter candidate. The Majorana mass term of these types of models is typically on the order of Grand Unified theories (GUT) scales $\left(10^{13}-10^{15} \mathrm{GeV}\right)[19,20]$. Whatever the scale, models of this nature would demonstrate physics beyond the standard model.

\subsubsection{Neutrinoless Double Beta Decay}

The smoking gun signature for Majorana neutrinos would be the observation of self-annihilation, in which two neutrinos annihilate to produce a photon. Attempting to discover neutrinos in this manner leads to a problem however, because neutrinos have a very low cross-section making it impossible to make an experiment in which neutrino self-annihilation can be observed directly. Instead, scientists are looking for evidence of a class of lepton number violating interactions in which neutrinos cancel internally. One such interaction would be neutrinoless double-beta decay.

It was shown by Schecter and Valle[21] that any observation of $0 \nu \beta \beta$ would imply the existence of a Majorana mass term. Neutrinoless double decay occurs under the same circumstances as for $2 \nu \beta \beta$ as outlined in section 3.1. The key difference is that no final state neutrinos are produced:

$$
d d \longrightarrow u u+2 e^{-}
$$

The mechanism that allows for this is the internal self-annihilation of the neutrinos. Since the Majorana mass term mixes the neutrino with its anti-neutrino, either in the case of equation 2.29 or in the case of 2.33, it allows for neutrinos to annihilate in the same manner as other fermions. Since neutrinos can annihilate, this also allows for processes to proceed by the exchange of virtual neutrinos, which is precisely what occurs in $0 \nu \beta \beta$. In this decay mode, the Majorana mass term of equation 2.29 indicates that the emission of an electron anti-neutrino with right handed helicity is equivalent to the absorption of a neutrino of left handed helicity. This is effectively the same as having a virtual line where the neutrinos cancel out due to self annihilation. The process itself is illustrated in figure 2.3. 


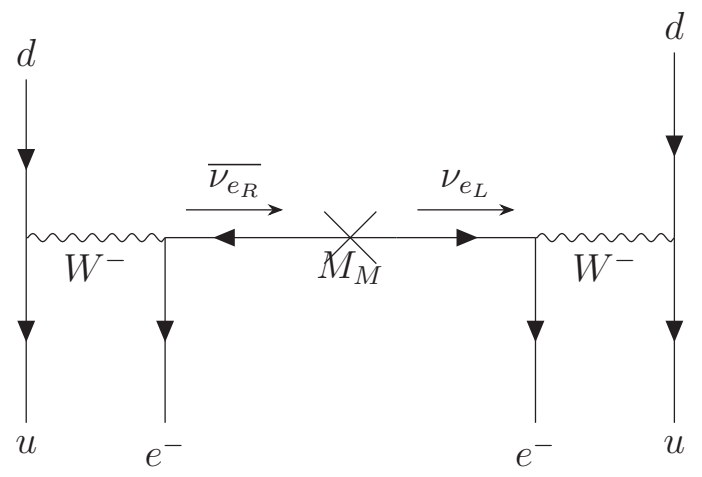

Figure 2.3: The leading diagram for neutrinoless double-beta decay. In this diagram, an electron anti-neutrino leaves one of the weak interaction vertices via the decay of a $W^{-}$. Since all neutrinos produced in weak decays as observed thus far are left handed and all anti-neutrinos observed so far are right handed, the antineutrino emitted in this vertex must be of right handed helicity. The Majorana mass term converts the outgoing right handed anti-neutrino into a left-handed neutrino, which then enters the other weak vertex. Due to the mixing from the Majorana mass, the absorption of a left handed neutrino is equivalent to the emission of a right-handed anti-neutrino. This diagram was once again drawn using the TikZ-Feynman package for LaTeX [1].

One may notice that this diagram does not cover the case in which the mass nature of the neutrino field is described by the Lagrangian in equation 2.33. In the case where a Dirac mass and Majorana mass are present the incoming neutrino must be of the same helicity of the emitted anti-neutrino in order for the vertex to cancel. The reason that inclusions of both mass terms still predict $0 \nu \beta \beta$ is explained nicely by Kayser in [2]. From either Lagrangian, the effect of the Majorana mass term is to change the particle into its anti-particle and invert the helicity. In [2] it is noted that the effect of a Dirac mass insertion is to reverse the helicity without changing from particle to anti-particle, thus satisfying the requirement that the neutrino entering the second vertex be right-handed. This effect is illustrated in figure 2.4, below. 


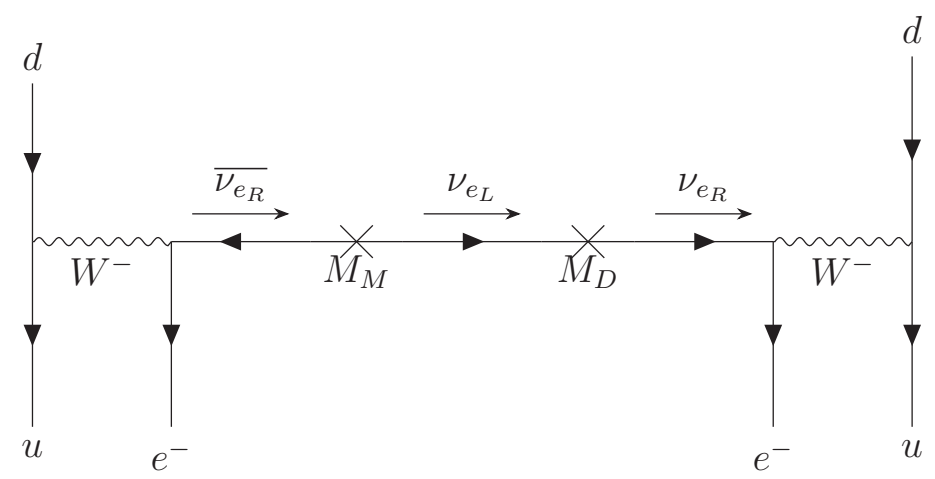

Figure 2.4: The Feynman diagram of figure 2.3 re-imagined for the case where the mass term in the Lagrangian for the neutrino field includes both Majorana and Dirac mass terms, based on the generalizations of [2]. Like in the diagram where the sole mass term was assumed to be Majorana in nature, the first weak vertex emits a right-handed electron anti-neutrino which is converted into a left handed neutrino by the insertion of a Majorana mass term. The handedness of the neutrino is then flipped again by the insertion of a Dirac mass term so that it can be absorbed in the second weak vertex, thus completing the virtual neutrino exchange. Like all of the Feynman diagrams in this thesis, this one was drawn using TikZ-Feynman [1].

What the inclusion of figures 2.3 and 2.4 are intended to demonstrate is that, regardless of whether neutrino fields contain the Majorana mass term alone or a Majorana term in addition to a Dirac term, one expects that neutrinoless doublebeta decay is a process that is observable, and further directly implies a Majorana mass term for the neutrino. Since there are no final state neutrinos in this decay mode, all of the available energy is shared between the recoiling nucleus and the final state electrons. This means that the complete energy can be reconstructed from the event since ions and electrons are observable in the correct type of detector. One would then expect, if neutrino-less events do occur in nature, that they would present themselves in the data as a bump in the energy spectrum for double-beta decay right at the $\mathrm{Q}$ value. The limiting factor on the detection of $0 \nu \beta \beta$, if it exists, is the amplitude of this bump with respect to the amplitude of two-neutrino events and other backgrounds.

The signal for $0 \nu \beta \beta$ decay ultimately comes down to its half-life, and thus the number of events that can be expected in a given volume and over a given time period. In explaining this, the literature $[18,22,6,23]$, defines a quantity $m_{\beta \beta}$ known as the effective Majorana mass for neutrinoless double-beta decay. This quantity is defined [6] as

$$
m_{\beta \beta}=\sum_{i=1}^{3} U_{e i}^{2} m_{i}
$$


where the terms $m_{i}$ are the mass eigenstates of the neutrino and the matrix $U_{e i}$ is the matrix $V$ from equation 2.32, which is the PMNS matrix which explicitly includes the theoretical Majorana phases. Casting this in terms of the Majorana CPV phases, this can be expanded in terms of the expected value of the effective Majorana mass for $0 \nu \beta \beta[18,10,22,23]$

$$
<m_{\beta \beta}>=\left|m_{1} \cos ^{2}\left(\theta_{12}\right) \cos ^{2}\left(\theta_{13}\right)+m_{2} \sin ^{2}\left(\theta_{12}\right) \cos ^{2}\left(\theta_{13}\right) e^{i \alpha_{21}}+m_{3} \sin ^{2}\left(\theta_{13}\right) e^{i \alpha_{31}}\right|
$$

noting that in the literature the Majorana CPV phases are often defined as $\alpha_{21}=\alpha$ and $\alpha_{31}=\beta$. The most general expression relating the half-life of neutrino-less double beta-decay is cited in the nEXO design report [18] as

$$
\left|T_{1 / 2}(0 \nu \beta \beta)\right|^{-1}=G^{0 \nu}\left|g_{A}^{2} M_{G T}^{0 \nu}-g_{V}^{2} M_{F}^{0 \nu}+g_{A}^{2} M_{T}^{0 \nu}\right|^{2}<m_{\beta \beta}>^{2} / m_{e}^{2}
$$

where $G^{\nu}$ is a factor arising from the phase space of the decay rate and the term in the absolute value is the expanded nuclear matrix element (NME) based on [24]

$$
M=M_{G T}-\frac{g_{V}^{2}}{g_{A}^{2}} M_{F}+M_{T}
$$

In equations 2.40 and 2.41 the $g_{V}$ and $g_{A}$ refer to the coupling strength of the weak force to vectors and axial vectors respectively, and the subscripts $G T, F$ and $T$ refer to the Gamow-Teller, Fermi and Tensor matrix elements respectively. The actual mechanics of the NME is outside of the scope of this thesis and are included for completeness. The main takeaway is that the NMEs are a significant source of uncertainty in the precise predictions of neutrino-less decay rates. However, under the assumption that the NME should be similar between two-neutrino and neutrino-less double-beta decays, equation 2.40 predicts that the half-life of $0 \nu \beta \beta$ should be proportional to $m_{e}^{2} /<m_{\beta \beta}>^{2}$. Perhaps more concisely, the half-life is inversely proportional to the square of the effective Majorana mass for $0 \nu \beta \beta$. This means that, based on equation 2.39, the half-life is sensitive to the individual neutrino masses. In particular, this half-life is very sensitive to what is known as the hierarchy or ordering of the neutrino masses. Hierarchy comes about because of the fact that only the absolute values of the mixing angles in neutrino oscillations are known, thus allowing for different orderings of the neutrino masses. This concept is a major motivation for the research presented in this thesis and is discussed in more depth in the following section.

Before diving into the neutrino mass hierarchy, it might be convenient to summarize the consequences of a potential discovery of neutrinoless double-beta decay. First, it demonstrates the existence of Majorana particles and a Majorana mass 
term in the field equations of the neutrino. It would further immediately demonstrate an example of a lepton number violating process which could be of importance in understanding the matter/anti-matter asymmetry in the early universe. Along the same theme, it would open the possibility of additional $\mathrm{CP}$ violating phases in the Lepton sector, which is similarly important towards understanding matter/anti-matter asymmetry. Finally, the discovery of Majorana neutrinos would potentially open up research into new physics as scientists try and understand whether neutrinos are Majorana alone in nature or contain a Dirac mass term as well.

\subsubsection{Neutrino Mass Hierarchy and Matter Effect in Neu- trino Oscillations}

As touched upon in the previous section, the neutrino mass hierarchy refers to the relative ordering of the neutrino mass states. Recall that neutrino oscillations are formulated in terms of the mass-squared differences between the mass eigenstates $\Delta_{m_{21}}^{2}, \Delta_{m_{32}}^{2}$ and $\Delta_{m 31}^{2}$. Since oscillation experiments can only practically measure the magnitudes of these mass differences, it leaves open the possibility for different orderings of the neutrino masses known as the inverted and normal hierarchies. The inverted hierarchy is defined as the scenario where

$$
m_{3}<m_{1}<m_{2}
$$

whereas the normal hierarchy is defined as the scenario where

$$
m_{1}<m_{2}<m_{3}
$$

Which hierarchy nature follows does have serious consequences for the required sensitivity of experiments seeking to detect $0 \nu \beta \beta$. As was explained in the previous section, the projected half-life for neutrino-less double-beta decay depends on the effective Majorana mass term , $\left\langle m_{\beta \beta}>\right.$. Based on equation 2.39, this

quantity is highly dependent on the individual masses of the neutrino mass states. Based on simulations $[25,22,26]$, an inverted hierarchy forces a very small effective Majorana mass, and thus a much longer half-life than is predicted for an inverted hierarchy scenario. Recall that nEXO was designed to probe the inverted hierarchy, translating to a half-life sensitivity of $10^{28}$ years corresponding to $m_{\beta \beta}=0.015 \mathrm{eV}$.

This simulation was initially presented in a paper by Francesco Vissani [26]. More updated versions are presented in a paper by Giovanni Benato [22] and in 
Reaching for the Horizon: The 2015 Long Range Plan for Nuclear Science [27] $]^{11}$ Based on these simulations, in the case of the inverted hierarchy, a sensitivity to an effective Majorana mass of $15 \mathrm{meV}$ would be sufficient to measure $0 \nu \beta \beta$ at a significance of $3 \sigma[18,25,27]$. In order to reach the $5 \sigma$ significance for discovery, the required sensitivity would be to an effective Majorana mass of $10 \mathrm{meV}$ [22]. In order to achieve a discovery in the normal hierarchy scenario, the required effective Majorana mass sensitivity is significantly smaller. Based on the 2015 paper by Benato, the $5 \sigma$ for a normal hierarchy scenario are reduced to below $1 \mathrm{meV}$, which would require significantly larger and more sensitive detectors.

This naturally leads into the question of why physicists might expect a normal hierarchy in the first place. In nature, there are measurable effects that are hierarchy dependent. Most noteworthy is the matter effect in neutrino oscillations. The formalism for this effect is derived in detail by Fantini et al [9] in their review of the formalism of neutrino oscillations. A simpler, more immediately usable result for experimentalists can be found in a paper awaiting publication from the Super-Kamiokande collaboration. In the treatment in their write up, they are primarily concerned with the oscillation probabilities $P\left(\nu_{e} \rightarrow \nu_{e}\right), P\left(\nu_{\mu} \rightarrow \nu_{\mu}\right)$ and $P\left(\nu_{\mu} \rightarrow \nu_{e}\right)[28]$. In the matter effect terms, the sine of the mixing angle and the mass splitting are replaced by their matter terms. The sinusoidal term is replaced by

$$
\sin ^{2}\left(2 \theta_{13}\right) \longrightarrow \frac{\sin ^{2}\left(2 \theta_{13}\right)}{\left(\cos \left(2 \theta_{13}-\frac{k 2 \sqrt{2} G_{F} N_{e} E_{\nu}}{\Delta_{m_{32}}^{2}}\right)^{2}+\sin ^{2}\left(2 \theta_{13}\right)\right.}
$$

and the mass splitting term is replaced by

$$
\Delta_{m_{32}}^{2} \sqrt{\left(\cos \left(2 \theta_{13}-\frac{k 2 \sqrt{2} G_{F} N_{e} E_{\nu}}{\Delta_{m_{32}}^{2}}\right)^{2}+\sin ^{2}\left(2 \theta_{13}\right)\right.}
$$

where $G_{F}$ is the Fermi constant, $N_{e}$ is the electron density of the material the neutrinos are travelling through, and $k$ is a factor with a value of \pm 1 depending on whether a neutrino is a neutrino or anti-neutrino. Clearly oscillations involving these quantities are dependent on the sign of of $\Delta_{m_{32}}^{2}$ and thus on whether $m_{3}>m_{2}$ or vice-versa. This is explicitly hierarchy dependent as it is known that $m_{1}<m_{2}$. The same paper reports on results that suggest a normal hierarchy and reject and inverted hierarchy at $74 \%$ [28]. The T2K collaboration similarly found a slight preference for a normal hierarchy [29]. Most notably, the NO $\nu$ A collaboration at

\footnotetext{
${ }^{11}$ This is also cited in the nEXO design report. The hyperlink for that reference among others does not appear to work any more. The US Department of Energy Office of Scientific and Technical Information does maintain a working url at https://www.osti.gov/biblio/1296778reaching-horizon-long-range-plan-nuclear-science.
} 
Fermilab measured neutrino oscillations favouring a normal hierarchy at a significance of $1.9 \sigma$ [30], which was published in June 2019. While these results are hardly conclusive, they do show that physicists need to prepare for the possibility of a normal hierarchy scenario and that it is prudent to begin planning for third generation experiments and for potential upgrades to tonne scale programs. 


\section{Chapter 3}

\section{Experimental Direction}

\subsection{A Brief History of Double-Beta Decay}

Double beta decay is a nuclear phenomenon originally characterized by German physicist and Nobel Laureate Maria Goeppert-Mayer in the mid 1930s [31] [32]. This type of decay occurs in isotopes where decay to the Z-1 beta daughter is energetically forbidden but not decay to the Z-2 daughter element. In these decays it is as if two beta decays happen simultaneously producing a pair of electrons, a pair of anti-neutrinos, and the daughter nucleus. Since there are two weak vertices in these processes ${ }^{1}$, the matrix element is suppressed so that the half-lives of double-beta candidate elements are extremely long, as can be seen empirically in [34] and [35]. Two-neutrino double-beta decay, commonly abbreviated $2 \nu \beta \beta$, was first observed by Elliot et al in 1987 for ${ }^{82} \mathrm{Se}$. The phenomenon has since been observed in many elements [34] and was first ${ }^{2}$ observed in ${ }^{136} \mathrm{Xe}$ in 2011 by the EXO-200 experiment [35], part of the Enriched Xenon Observatory (EXO). There are several analogous second-order processes, such as the recently observed twoneutrino double electron-capture recently observed by the XENON dark matter collaboration [36]. The rest of this thesis will focus specifically on double-beta decay and specifically on xenon-136.

Later in the 1930s Majorana [37] suggested a model in which neutrinos could be their own anti-particles which was later expanded by Racah [38]. Furry [39] then interpreted the Majorana theory as suggesting a possible neutrinoless doublebeta decay, predicting singnificantly shorter half-lives than Goeppert-Mayer [32]. More recently, the current understanding of electroweak physics has been used to show that $0 \nu \beta \beta$ requires both that neutrinos have mass and that they have a Majorana mass term in their field equations [21]. The observation of neutrino

\footnotetext{
${ }^{1}$ This is referred to in the literature as a "second order weak process", for example in [33]

${ }^{2}$ Private correspondence with Prof. Thomas Koffas labels this paper as the discovery of $2 \nu \beta \beta$ in Xenon. The timeline in [34] seems to corroborate this.
} 
flavour oscillation in atmospheric neutrinos by Super Kamiokande [8] and the subsequent confirmation from solar neutrino data by the SNO collaboration [7] and from nuclear reactors in the KamLAND long baseline experiment [40] have confirmed beyond doubt that neutrinos are massive.

There are several reasons why physicists are interested in detecting neutrinoless events. The first of these is simply to evaluate the nature of neutrinos. Currently, all standard model fermions whose nature is known are Dirac particles, meaning that they have distinct particle and anti-particle states. Observing $0 \nu \beta \beta$ decays would be a smoking gun discovery, and would be the first confirmation that Majorana particles do exist in nature. There is already some indication that neutrinos could be Majorana in nature. As it stands, all observations of weak interactions involving neutrinos are left handed ${ }^{3}$, referring to the projections of weak couplings given by

$$
(L, R)=\frac{1 \pm \gamma_{5}}{2}
$$

from the standard theory of weak interactions [4], where $\gamma_{5}$ is the pseudoscalar Dirac matrix [5], and the operators $\mathrm{L}$ and $\mathrm{R}$ project an interaction into left and right handed chirality states. ${ }^{4}$ These results are typically taken to mean the right handed neutrino does not couple to the weak bosons ${ }^{5}$ [41] in the Standard Model. A tempting explanation for this is that there are no right-handed neutrino states or left-handed anti-neutrino states [11], which could certainly be true in the case of Majorana neutrinos.

Neutrinos are also of significant importance at much larger scales, in Big Bang Cosmology. In the Standard Model matter and anti-matter are formed by pair production [5, 4], illustrated as

$$
\gamma \longrightarrow f+\bar{f}
$$

Due to the symmetry of equation 3.2, one would expect equal amounts of matter and antimatter to be produced. The timescale for recombination suggests that this would have happened already and our universe would be completely light dominated ${ }^{6}$. This is not the case however, and we observe that ordinary matter still accounts for a small fraction of the energy content in the universe ${ }^{7}$. The-

\footnotetext{
${ }^{3}$ The author first heard the argument expressed in this way in a lecture on quantum field theory as part of a graduate level course on the subject, taught by Prof. Daniel Stolarski. The argument is perhaps made more clear by statements in [11] and [41]

${ }^{4}$ What is meant by projections, Dirac matrices, and chirality are topics for chapter 2 which goes into more detail on the physics of Neutrinos.

${ }^{5}$ Nor do the left-handed anti-neutrinos

${ }^{6} \mathrm{~A}$ common statement in cosmology and $0 \nu \beta \beta$ themed talks at the physics department colloquia.

${ }^{7}$ From a graduate level lecture in particle physics by Prof. Bruce Campbell. The lecture also
} 
orists suggest that this problem is overcome by introducing a small asymmetry between matter and antimatter, a model known as baryogenesis [42]. The conditions allowing for this are known as the Sakharov [43] conditions $^{8}$. One of these conditions is violation of lepton number and baryon number conservation. Lepton and baryon number are both observed symmetries in particle physics, however no physical reason has been given as to why they are symmetries. The $0 \nu \beta \beta$ decay is by its very nature lepton number violating. Lepton number violation is also in and of itself a motivation to look for neutrinoless events. It has also been proposed [44] that sufficient matter-antimatter asymmetry is produced by a Majorana mass term for the neutrinos to allow for the necessary baryon asymmetry.

Neutrinoless double-beta decay also provides some potential for probing of the neutrino mass scale. The expected half-life of $0 \nu \beta \beta$ is usually given in the form of equation 3.3 [25].

$$
\left(T_{1}^{0 \nu} / 2\right)^{-1}=G^{0 \nu}\left|M^{0 \nu}\right|^{2} \frac{<m_{\beta \beta}>^{2}}{m_{e}^{2}}
$$

Here the term $G^{0 \nu}$ is the phase space factor [23] [25], $M^{0 \nu}$ is the nuclear matrix element (NME) taking into account all nuclear effects, and $\left\langle m_{\beta \beta}>\right.$ is the effective Majorana mass term. The nuclear matrix element is typically cited as difficult to calculate and there are several methods to approximate this. The effective Majorana mass term is parametrized in terms of the neutrino masses and is often reported in the form of equation 3.4 [18]

$$
\begin{array}{r}
<m_{\beta \beta}>=\left|\sum_{i=1}^{3} U_{e i}^{2} m_{i}\right| \\
=\left|m_{1} c_{12}^{2} c_{13}^{2}+m_{2} s_{12}^{2} c_{13}^{2} e^{i \alpha}+m_{3} s_{13}^{2} e^{i \beta}\right|
\end{array}
$$

where the $U_{e i}$ terms are elements of the PMNS matrix from the previous chapter, which mixes neutrino states. This matrix is given in [18] as

$$
U=\left(\begin{array}{ccc}
c_{12} c_{13} & s_{12} c_{13} & s_{13} e^{-i \delta_{C P}} \\
-s_{12} c_{23}-c_{12} s_{23} s_{13} e^{i \delta_{C P}} & c_{12} c_{23}-s_{12} s_{23} s_{13} e^{i \delta_{C P}} & s_{23} c_{13} \\
s_{12} s_{23}-c_{12} c_{23} s_{13} e^{i \delta_{C P}} & -c_{12} s_{23}-s_{12} c_{23} s_{13} e^{i \delta_{C P}} & c_{23} c_{13}
\end{array}\right)
$$

Where the sine and cosine terms are abbreviated as the letters "s" and " $c$ " with subscripts corresponding to the relevant mixing angles $\theta_{12}, \theta_{23}$, and $\theta_{13}$. Equations 3.3 and 3.4 are typically interpreted to mean that the half-life of $0 \nu \beta \beta$ is dependent

introduced the Sakharov conditions

${ }^{8}$ The DOI for the citation is for a reprinting of the article appearing in 1991 . Only the reprinting has a DOI available. 
on the mass of the lightest neutrino and the neutrino hierarchy. In particular, it is implied that in the case of a normal hierarchy the effective Majorana mass is dependent on the mass of the lightest neutrino [45]. Current proposed tonne-scale detectors are looking to probe in the region of $\mathrm{T}_{1 / 2}=\mathrm{O}\left(10^{28}\right)$ years [24]. If the hierarchy of neutrino masses does indeed follow the normal hierarchy, in which the electron neutrino is the lightest, then it raises the expected half-life to greater than this sensitive region [23] and thus more sensitive detectors are needed. As it stands, the half-life for $0 \nu \beta \beta$ is suppressed by the virtual neutrino vertex $[6,24]$.

Since the $0 \nu \beta \beta$ cross-section is even smaller than for $2 \nu \beta \beta$ decays, it necessitates even more sensitive detectors. The required sensitivity of any underground physics detector is dependent on how rare the process is and the background processes affecting the signal. It has already been discussed that neutrino-less double beta decay is by definition a very rare process. While, based on the projections of [23], next generation experiments will meet or exceed[25] the required sensitivity for inverted hierarchy expectations, the possibility of normal hierarchy neutrino masses cannot be ignored.

The backgrounds for double-beta experiments are typically characterized in terms of those that are expected to be present and expected to interfere with experimental measurements. These are somewhat dependent on the experimental setup, therefore the discussion here will focus on the backgrounds of concern for the proposed nEXO due to similarities in described experiment ${ }^{9}$. The nEXO collaboration projects [25] three main background sources in addition to the obvious two-neutrino channel for double-beta decay. These ${ }^{10}$ are $\gamma$ and $\beta$ emitting radionuclides present in detector materials, radio-contaminants resulting from interactions with cosmic rays, and ${ }^{222} \mathrm{Rn}$ which produces ${ }^{214} \mathrm{Bi}$ in its decay chain. The latter is particularly dangerous as the signature $\gamma$ for the decay of ${ }^{214} \mathrm{Bi}$ is at $2448 \mathrm{keV}$ [25] which is close to measurements for the Q-value of double- $\beta$ in Xe which Redshaw et al [46] measured at $(2457.83 \pm 0.37) \mathrm{keV}$ and McCowan and Barber [47] measured at (2458.7 \pm 0.6$) \mathrm{keV}^{11}$.

This thesis discusses a potential improvement to a nEXO type experiment. This is specifically the use of a two-phase design rather than a liquid medium $t p c$, which would potentially be able to reject several backgrounds. The structure of event tagging in nEXO has events split into single and multi-site events [25]. Of the multi-site events only a very small fraction are expected to be can-

\footnotetext{
${ }^{9}$ The nEXO experiment is a proposed xenon-136 TPC and is discussed in the next section as part of an overview on $\nu \nu \beta \beta$ searches in xenon. This is similar to the author's experimental set-up which is a two-phase xenon TPC, a type of detector which is described in more detail in chapters 4 and 5. The author is also a member of the EXO collaboration.

${ }^{10}$ See table II in [25].

${ }^{11}$ The nEXO collaboration weights these results and cites the Q-value of double-beta in Xe as $(2458.07 \pm 0.31) \mathrm{keV}$ in $[25]$.
} 
didate double-beta events ${ }^{12}$. A goal of any double-beta experiment is to reduce the backgrounds to just the two-neutrino channel of target isotope. The nEXO collaboration quantifies the effect of this reduction in $[25]^{13}$. First of all, it would reduce the number of background events in the range of the double-beta Q-value \pm one half of the full width half maximum by a factor of 100 . It would also bring the $2 \sigma$ resolution and $3 \sigma$ discovery potential above the $10^{28}$ year threshold.

There is an obvious advantage even for current scale experiments to implement technology that reduces backgrounds to just $2 \nu \beta \beta$. For example, while nEXO can entirely probe the inverted hierarchy at $3 \sigma$, it would need to become more sensitive still to be able to claim discovery. A two-phase TPC is potentially such an improvement. This thesis will discuss the implementation of a prototype twophase xenon TPC. The advantage of such a setup would be to discriminate based on pulse shape between one and two electron events. A veto system capable of achieving this would be able to pre-select for events single site events with two electrons originating from the same vertex, eliminating single $\gamma$ and $\beta$ background events.

\subsection{Review of Double-Beta Searches}

The aim of this section is to briefly mention the current scientific endeavours looking for $0 \nu \beta \beta$ in ${ }^{136} \mathrm{Xe}$ as well as other nuclei with particularly advanced research programs. It will very generally go over the basics of their set-up and methodology, as well as any results measured by the mentioned collaborations. As it stands, there are four currently deployed or recently finished experiments in EXO-200, KamLAND-Zen, XMASS and NEXT and three proposed experiment in nEXO, KamLAND2-Zen, and PandaX-III. Two of the proposed programs, nEXO and KamLAND2-Zen are upgrades to experiments that recently finished their data taking runs. The scope of this section will be limited to the most relevant of these, EXO-200, KamLAND-Zen as well as nEXO.

\subsubsection{EXO-200 and nEXO}

The set up of the EXO-200 detector is described in detail in [48] and a number of upgrades implemented after a two-year shut down are detailed in [49]. EXO200 was a liquid xenon TPC located at the Waste Isolation Pilot Plant (WIPP) outside Carlsbad, New Mexico. EXO-200 was specifically a double-TPC, so that there were two cylindrical volumes placed side-by-side. WIPP has an overburden

\footnotetext{
${ }^{12}$ See FIG 7 in [25].

${ }^{13}$ Shown in FIG 14 in that publication
} 
of 1585 meters water equivalent as measured by Esch et al[50]. The EXO-200 detector consisted of $110 \mathrm{~kg}$ active mass of liquid xenon enriched to $80.6 \%{ }^{136} \mathrm{Xe}$ [48]. Events are reconstructed using an ionization channel and a scintillation channel, based upon the principle the energy deposited can be reconstructed more accurately based on the anti-correlation of scintillation and ionization signals [51]. Scintillation light is collected by an array of large area avalanche photo-diodes (LAAPDs), while the charge is collected by a criss-crossing pattern of wires [48]. The electric field inside the active region is shaped to achieve a uniform electric field [48]. Finally, the spacing of the high voltage and virtual ground wires are set-up so as to give 3-d position resolution of electron tracks in the TPC[48]. This was done with the goal of distinguishing between single and multi-site events. As mentioned in the introduction, the EXO-200 collaboration first measured the half life of $2 \nu \beta \beta$ in ${ }^{136} \mathrm{Xe}$ to a value of $2.11 \pm 0.04$ (stat) \pm 0.21 (syst) $\times 10^{21}$ years in $2011[35]$. In 2014 the collaboration reported a then best in the world ${ }^{14}$ lower limit of $1.1 \times 10^{25}$ years on $0 \nu \beta \beta$ at the $90 \%$ confidence level [52]. With the increased shielding and refined fitting techniques detailed in [49], this limit was increased to $1.8 \times 10^{25}$ years.

The next generation of the EXO collaboration's search for $0 \nu \beta \beta$ is the nEXO detector, a proposed five-tonne liquid xenon TPC [18]. The choice of increasing the liquid xenon mass from 200-kg all the way up to five-tonne was based on a calculation of the required mass to achieve a minimum one count per year event rate for a half-life of $\mathrm{T}_{1 / 2}=9.7 \times 10^{27}$ year [18]. As discussed previously this is the order of sensitivity required to probe the inverted mass hierarchy regime. The differences in design between nEXO and EXO-200 largely have to do with overcoming challenges relating to the massive scaling-up, as well as upgrades in technology that would not have been possible for the EXO-200 detector. Some of these improvements include, from the nEXO collaboration design report, [18]

- Eliminating the charge collection wires and replacing them charge collecting pads, thus eliminating the need for a higher electric field region around the wire grid. In EXO-200 the wires ran through the detector, in nEXO charge sensitive pads are at the end of the chambers. This also reduces the material in the detector itself.

- The nEXO detector is a single long chamber, as opposed to the double TPC design of EXO-200. The use of a single homogeneous volume allows the experiment to take advantage of xenon self-shielding. Essentially, due to its high stopping power, many background events near the surface of the xenon

\footnotetext{
${ }^{14}$ Based on email correspondence from T. Koffas. Supported by the fact that the result was first published in Nature.
} 
are stopped well before reaching the inner volume of the detector.

- Charge collection was changed from the collection wires to fused-silica pads with metal contacts deposited on their surface. The goal of this was to take advantage of the increased stability of these tiles, as wires are susceptible to vibrations, in addition to taking advantage with ease of assembly.

- nEXO also makes improvements to the other component of the dual readout, by changing the light readout to silicon photomultiplier (SiPM). Silicon photomultipliers are in general faster and more sensitive than LAAPDs, such as those used in EXO-200. The characterization of these devices for use in $\mathrm{nEXO}$ is reported in [53].

The nEXO detector also has many of the same requirements as EXO-200. These include for highly radiopure detector components as well as continuous purification of Xenon to take advantage of the anti-correlation between scintillation and charge signals. The target discovery potential of $\mathrm{nEXO}$ is $\mathrm{T}_{1 / 2}=5.7 \times 10^{27}$ years at a significance of $3 \sigma$, with a minimum lower bound of $\mathrm{T}_{1 / 2}=9.2 \times 10^{27}$ years at 90\% confidence [25]. Upgrades during the running life are expected to bring the sensitivity of nEXO up above a sensitivity of $10^{28}$ years [25].

\subsubsection{KamLAND-Zen}

KamLAND-Zen stands for Kamioka Liquid Scintillator Antineutrino Detector Zero-Neutrino Double-Beta Decay and is one of several experiments located at the Kamioka Mine in Japan. The KamLAND-Zen collaboration currently has the best limit in the world on $0 \nu \beta \beta$ decay with a lower limit of $T_{1 / 2}=1.06 \times 10^{26}$ years at $90 \%$ confidence [54], for which there has yet to be a better claimed result as of $2019^{15}$. The KamLAND-Zen detector is a double-walled balloon structure adapted from the original KamLAND detector [55]. The inner balloon consists of liquid scintillator doped with Xenon enriched to approximately $2.5 \%{ }^{136} \mathrm{Xe}$, amounting to $300 \mathrm{~kg}$ of the active isotope [55]. The record-setting result cited from [54] states that the Xenon content of the inner balloon liquid scintillator was increased to $2.9 \%$. The liquid scintillator contained in the outer balloon has a density near that of the inner scintillator [55]. The high density of the detection media is paired with a thick stainless steel tank to help provide shielding. KamLAND-Zen is unable to track individual electrons produced in decays, and so only records the combined energy of electrons released in candidate events[55, 54]. The light readout is done by 1325 photomultiplier tubes[55], which also track the vertex of the event.

\footnotetext{
${ }^{15}$ To the authors knowledge
} 


\subsubsection{CUORE}

The Cryogenic Underground Observatory for Rare Events (CUORE) collaboration uses a bolometer technique to search for neutrino-less double-beta decay in tellurium-130, another double-beta candidate. This technique uses $\mathrm{TeO}_{2}$ crystals as both the detector and isotope source [56]. The basic idea behind this technique is that $\mathrm{TeO}_{2}$ has an exceptionally low heat capacity at low temperature so that particle interactions in the decay produce a subsequent rise in temperature that is measurable [56]. This temperature rise can then be connected to the energy of the final state electrons. CUORE cools its crystals down to $10 \mathrm{mK}$, which is achieved through the use of a dilution refrigerator [56]. The detector itself consists of an array of 988 bolometers arranged in 19 columns with a total active mass of $206 \mathrm{~kg}$ [56]. Most recently, the collaboration measured the double-beta decay of ${ }^{130} \mathrm{Te}$ to the first excited state of ${ }^{130} \mathrm{Xe}$. The results for each of the decay modes with $90 \%$ confidence were half lives of $\mathrm{T}_{1 / 2}>2.4 \times 10^{23}$ years and $\mathrm{T}_{1 / 2}>7.9 \times 10^{23}$ for each of the two-neutrino and neutrino-less cases respectively [57]. CUORE has also measured the half-life of the two-neutrino mode in ${ }^{130} \mathrm{Te}$ in the decay to ground state Xe with a half life of $\mathrm{T}_{1 / 2}=(7.9 \pm 0.1$ (stat. $) \pm 0.2$ (syst) $) \times 10^{20}$ years [58].

\subsubsection{Germanium Experiments}

It is worth mentioning as well the experimental program in Germanium-76, which is another double-beta decay candidate. The two key experiments in this area are the MAJORANA collaboration and the GERDA collaboration. The MAJORANA collaboration uses an array of high-purity germanium (HPGe) detectors, which are enriched so that they provide both the source material as well as the detection method [59]. As of 2019, the MAJORANA DEMONSTRATOR contained 58 HPGe detectors in two modules with a total mass of $44.1 \mathrm{~kg}$, of which $29.7 \mathrm{~kg}$ have been enriched to $88 \%{ }^{76} \mathrm{Ge}[59]$. The DEMONSTRATOR also employs an active muon veto system [59]. So far, the MAJORANA demonstrator has claimed a lower limit of $1.9 \times 10^{25} \mathrm{yr}$ with a confidence of $90 \%$ [60]. GERDA, which stands for Germanium Detector Array, is a similar experiment to MAJORANA. As in MAJORANA, GERDA uses ${ }^{76} \mathrm{Ge}$ both to construct the detector as well as to provided the source of double-beta events, and consists of $35.6 \mathrm{~kg}$ enriched to $85 \%{ }^{76} \mathrm{Ge}[61]$. The array is submerged in liquid argon, scintillation light from which is picked up by photomultiplier tubes and allows for the rejection of more than $95 \%$ of background events [61]. The collaboration reported their best lower limit in Science in 2019 as $0.9 \times 10^{26}$ at $90 \%$ confidence [61]. In both GERDA and MAJORANA, the advantages for using germanium are the well known methods of constructing detectors with inherently high energy resolution and the low 
background region this allows them to operate in $[59,61]$. 


\section{Chapter 4}

\section{Scintillation in Noble Gases}

\subsection{Simple Processes in Signal Generation}

Noble Gas elements are generally prized for their properties as detector media. Specifically, they are prized for the fact that they are non reactive and have favourable optical properties when exposed to ionizing radiation. The heavier of these materials (Argon, Krypton, Xenon) are most commonly used as detector materials for low background physics experiments. This is because of their scintillation properties and, in the case of xenon, high stopping power. In addition, in $0 \nu \beta \beta$ experiments, enriched xenon has the additional advantage of providing both the detector and source material.

Scintillation is the tendency of these materials to release light when excited by incoming ionizing particles. Detectors may be constructed out of noble gas materials due to the fact that they, like other scintillators, are mostly transparent to the light they release under processes that generate scintillation light [62]. This effect is largely due to the fact that excited states produced via the deposition of energy from incoming particles tend to take the form of excited dimers, what Aprile and her co-authors describe in their book Noble Gas Detectors [62] as the molecular continuum of noble gas states. The light from radiative decays of excited dimers of xenon, usually called excimers, has a long attenuation length in the scintillating material. The wavelength of xenon scintillation is given as $178 \pm 1$ nm [63].

Ionization occurs when the incoming radiation deposits enough energy to remove an electron from the shell of an atom of the detector material. Since the energy scales in particle physics experiments are very large, these types of particles typically scatter many times and produce many ionizations. Multiple ionizations are also a possibility. This process then produces electron ion pairs. A single 
ionization event is described below in equation 4.1 below.

$$
A+x \longrightarrow A+e^{-}+y
$$

Where $A$ represents the atom of the detector medium (for example Xe), $x$ represents any particle of incoming radiation, and $y$ represents any particle of outgoing radiation produced by the interaction. Note that $y$ may represent multiple particles or $y$ and $x$ may be the same, as in the case of a scattering event, or there may be no outgoing particle, as in the case of photoelectric absorption (among other possible interactions). Over time, the electron ion pairs recombine into neutral atoms. Once recombination occurs, the final state atoms are in an excited state. The excited atoms de-excite releasing light which can be collected, as represented in equation 4.2 .

$$
A+e^{-} \longrightarrow A^{*} \longrightarrow A+\gamma
$$

Note that here a ${ }^{*}$ represents an excited state. The intermediate $A^{*}$ represents the excited detector atom that then decays radiatively to the ground state. Of course, the medium is not transparent to atomic transitions and this photon may eventually be absorbed by other atoms. For a medium that is sufficiently dense, the time for re-absorption of the atomic transition photon is short enough such that the photons may become trapped, forcing the excited atoms to collide and form excited molecular states [62]. The decay of these states releases photons of the wavelength of the molecular continuum, and is shown in equation 4.3 shown below.

$$
A_{2}^{*} \longrightarrow 2 A+\gamma_{m c}
$$

This type of excitation is referred to as a self-trapped excimer. It is generally accepted that the production of self-trapped excimers can also be produced by the direct excitation of atoms, as shown in equation 4.4 [64] [65] [66].

$$
A^{*}+A+A \longrightarrow A_{2}^{*}+A
$$

Note that Aprile and Doke [64] explicitly replaces the place holder $A$ with $X e$. The decay of this state then proceeds as shown in equation 4.3. Self-trapping effects are described in more detail by Martin [67].

Another more complicated process may be used to describe ionization and recombination in liquid-noble gases. This process is presented in works by Aprile 
and Doke [64] [66] in the form of equation 4.5.

$$
\begin{array}{r}
A^{+}+A \longrightarrow A_{2}^{+} \\
A_{2}^{+} e^{-} \longrightarrow A^{* *}+A \\
A^{* *} \longrightarrow A^{*}+\text { heat } \\
A^{*}+A+A \longrightarrow A_{2}^{*}+A \\
A_{2}^{*} \longrightarrow 2 A+\gamma_{m} c
\end{array}
$$

A related group of processes is also presented slightly differently in Noble Gas Detectors [62] and is reproduced here in equation 4.6.

$$
\begin{array}{r}
A^{+}+e^{-} \longrightarrow A^{*} \\
A^{+}+2 A \longrightarrow A_{2}^{+} A \\
A_{2}^{+} e^{-} \longrightarrow A^{* *}+A \\
A^{* *} \longrightarrow A^{*}+\text { heat }
\end{array}
$$

Note that the process in equation 4.6 assumes that there is no external electric field applied. The ionization and recombination schemes shown in equations 4.6 and 4.5 both rely on multiple collisions between ions and excited states of atoms or interactions between excitons, electron hole bound states that are also produced in events. The three main processes that contribute to scintillation light in liquid noble gases are outlined nicely as well by Doke in [68].

Excited dimers can be in either a triplet or singlet state, each of which have different decay times. This fact will be used to explain the timing structure of scintillation events in the following section. The last topic to touch on for an introduction is the origin of these two different states. Usually in the literature a singlet state is denoted as ${ }^{1} \Sigma_{u}^{+}$and a triplet state is denoted as ${ }^{3} \Sigma^{+} u$, so these conventions will be kept here. The literature attributes the triplet state to the direct excitation of atoms and self-trapped excitons [65] [67]. The singlet state then arises out of the recombination of ions and electrons [65] [69]. Kubota et al [65] refer to the later as "recombination luminescence" and describe a finite rise time. It would appear that this effect limits the maximum intensity that can be obtained by events with high populations of ${ }^{1} \Sigma_{u}^{+}$[65] [3]. There is also some evidence that the direct excitation of atoms that become self-trapped can populate the ${ }^{1} \Sigma_{u}^{+}$state to a degree [70]. Since the intensity of light emitted by 
singlet and triplet states each have different decay times, this sets up a discussion of the timing structure of noble gas scintillation.

\subsection{Timing Structure of Scintillation Events}

As described in the previous section, two different excited states are formed when liquid noble gases are exposed to ionizing radiation. Since each of these has a different lifetime, event structures tend to take the form, in the simplest case, of the function described in equation 4.7.

$$
\Sigma I(t)=a_{f} e^{-t / \tau_{f}}+a_{s} e^{-t / \tau_{s}}
$$

Equation 4.7 is a sum of exponential terms, with the $a$ terms representing the weighting constant for each component, and the $\tau$ terms representing the corresponding time constants. The subscripts $f$ and $s$ refer to the exponential decay created due to the fast and slow timing components respectively. The weighting constants and time constants can be measured by curve fitting functions of the form of equation 4.7 to histograms of light collection events. Note that the intensity, $I$, is in arbitrary units.

Another model that has been used to described the decay curve of scintillation light in noble liquids is a modification of Gale's [71] equation. The modification used appears in Noble Gas Detectors [62], Kubota et al (1978) [70], Hitachi et al (1983) [3] and Akimov et al (1996) [72]. All four representations should be the same, for clarity however the expanded form used by Hitachi [3] is included here with the explicit dependence changed to reflect the time constant rather than the decay time, as it is more useful for the type of analysis presented in this work.

$$
\begin{array}{r}
I(t)=A\left(\exp \left(\frac{1}{4 h^{2} \tau^{2}}-\frac{t}{\tau}\right)\left(1+\operatorname{erf}\left(h t-\frac{1}{2 h \tau}\right)\right)\right. \\
\left.-\exp \left(\frac{1}{4 h^{2} \tau_{w}^{2}}-\frac{t}{\tau_{w}}\right)\left(1+\operatorname{erf}\left(h t-\frac{1}{2 h \tau_{w}}\right)\right)\right)+ \text { baseline }
\end{array}
$$

where $A$ denotes the amplitude of the scintillation, $\tau$ denotes the decay time of the liquid scintillator, $\tau_{w}$ denotes the decay time of a wavelength shifting agent, and $\mathrm{h}$ is a statistical term taken to be $1 / \sqrt{2} \sigma$. In this case, the statistical spread, $\sigma$, is taken to be the resolution of the light collection device. Based on the original paper [71], the wavelength shifting term typically takes into account any window treatment required to shift the wavelength of light to the range of sensitivity of an available photomultiplier tube. Since this experiment uses a SiPM directly 
coupled to the gas there may be no need for wavelength shifted terms, reducing equation 4.8 to equation 4.9 below.

$$
I_{i}(t)=A\left(\exp \left(\frac{1}{4 h^{2} \tau_{i}^{2}}-\frac{t}{\tau_{i}}\right)\left(1+\operatorname{erf}\left(h t-\frac{1}{2 h \tau_{i}}\right)\right)\right)
$$

Note here that Aprile's [62] convention has been adopted so that time constants now have the subscript $i$. This is to indicate that a process in which multiple scintillation components of different lifetimes can be combined as in the case of the simple exponential

$$
\sum_{i=1}^{n} a_{i} I_{i}(t)
$$

where $n$ is the total number of lifetime components. Equation 4.10 also generates equation 4.7 when the intensity curves , $I_{i}(t)$ are taken to be the exponentials and the indices $i=1$ and $i=2$ are taken to be the fast and slow components of the scintillation light respectively.

Equation 4.8 is formulated differently in Noble Gas Detectors [62] as well as Akimov et al [72]. Applying this reformulation to the the simplified form of equation 4.9 gives

$$
I_{i}(t)=a_{i} e^{\left(\frac{1}{2}\left(h^{\prime} \tau_{i}\right)^{2}-\frac{t}{t a u_{i}}\right)}\left(1+\operatorname{erf}\left(h^{\prime} t-\frac{1}{2} h^{\prime} \tau_{i}\right)\right)
$$

where the modified term $h^{\prime}$ is now equivalent to $\frac{1}{\sqrt{2}} \sigma$. Note that as the in the limit where the time resolution of light collection becomes very small, equation 4.11 reduces to the simple exponential. In other words, for a collection device with a very fast response time and without a wavelength shifting medium or admixture one would expect the more simple case to describe the intensity curve of the scintillation light sufficiently. Since it may very well be the case that the simple exponential formulation of scintillation light may accurately describe the scintillation of liquid xenon in this experiment, the discussion of Gale's equation is included for completeness.

From the material in the previous section, a scintillation event [62] will produce a number photons given by

$$
N_{s c i}=N_{i}+N_{e x}=N_{i}\left(1+\frac{N_{e x}}{N_{i}}\right)
$$

where $N_{e x}$ and $n_{i}$ are respectively the total number of excited states produced by the deposition of energy by an ionizing particle and the total number of electron ion pairs. At this point, it is useful to define the quantities $W_{i}$ and $W_{s c}$. $W_{i}$ is 
defined as the minimum energy in $\mathrm{eV}$ required to produce an electron ion pair in a material. $W_{s c}$ is similarly defined as the minimum energy, again in eV, required to produce one scintillation photon. A convenient substitution can be made by use of equation 4.13 [66].

$$
W_{s c}=\frac{E}{N_{s c}}
$$

Note that the expression for $W_{i}$ takes the same form. Substituting equation 4.12 into equation 4.13 as in [62], one produces the following result for the value of $W_{s c}$.

$$
\begin{array}{r}
W_{s c}=\frac{E}{N_{i}}\left(\frac{1}{1+\frac{N_{e x}}{N_{i}}}\right) \\
W_{s c}=\frac{W_{i}}{1+\frac{N_{e x}}{N_{i}}}
\end{array}
$$

The form of equation 4.14 is convenient due to the fact that $W_{i}$ is an easily measured quantity. Indeed, this quantity has been measured by Takahashi et al as $15.6 \pm 0.3 \mathrm{eV}$ [73]. Noble Gas Detectors also reports on a direct measurement of $\mathrm{W}_{s c}$ with $13.8 \pm 0.9 \mathrm{eV}$.

A key takeaway of this discussion of the number of scintillation photons produced in an interaction is that it is fixed by equation 4.13 for a particular energy. In other words different species of ionizing radiation that deposit similar energies would produce a similar number of scintillation photons. This raises the question of how one might attempt to distinguish between different types of radiation.

In the previous section, the existence of two distinct states $\left({ }^{1} \Sigma_{u}^{+},{ }^{3} \Sigma_{u}^{+}\right)$produced in excitations of the liquid xenon molecular continuum was discussed. These states are populated by the different processes described in the previous section. Since the ${ }^{1} \Sigma_{u}^{+}$and ${ }^{3} \Sigma_{u}^{+}$states each have different lifetimes, even if an event deposits the same amount of energy, the shape of the scintillation pulse also depends on which processes to which that energy is distributed. This concept is perhaps best illustrated in a result from Hitachi et al [3], shown in figure 4.1. In this figure, it is clear that the overall lifetimes of scintillation pulses in liquid xenon due to different types of radiation varies greatly. This is due to the fact that different types of radiation distribute energy differently to different processes. 


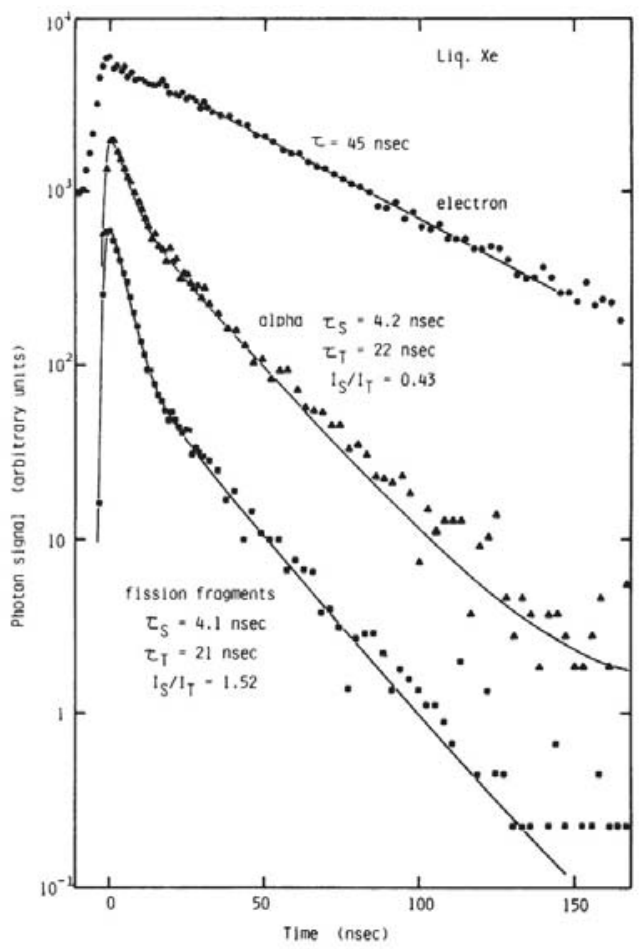

Figure 4.1: Scintillation events for relativistic electrons, $\alpha$-particles and fission fragments. Despite each type of radiation having similar energy, the scintillation profile is very different. This is because of how each type of radiation distributes deposited energy among processes that populate the ${ }^{1} \Sigma_{u}^{+}$and ${ }^{3} \Sigma_{u}^{+}$states. Electrons have a long tail, suggesting the interaction is dominated by self-trapping. Fission fragments and $\alpha$-particles each suggest a mix of self-trapping and recombination heavy processes are responsible for the resulting pulse shapes. The graph also gives measurements of the singlet and triplet lifetimes in liquid Xenon as well as the ratios of these states for different types of radiation. Curiously, note that a greater proportion of the signal seems to be lost for more ionizing types of radiation. This figured was reproduced from [3] and is used with permission. (C) American Physical Society. DOI: https://doi.org/10.1103/PhysRevB.27.5279.

The most interesting result shown in figure 4.1 can be found in the differences in pulse-shape of $\alpha$-particles and fission fragments. Note that in 4.1 the ratio of singlet to triplet states is included, being 0.43 and 1.52 for $\alpha$ 's and fission fragments respectively [3]. The main difference between how these two types of particles deposit energy in a medium is range. Both are multiply charged nuclei. Fission fragments however have a higher mass and higher charge and so the length they will travel on average in a medium is smaller. Defining the ionization density of an interaction

$$
\rho_{I}=N_{I} /<x>
$$

where $N_{I}$ is the number of electron ion pairs and $x$ is the distance travelled into 
the medium. Here the use of $\rho$ suggesting a three dimensional density is justified based on the idea that ionization follows a columnar distribution as first proposed by Jaffé in $1913^{1}$ [74] and later applied to liquefied gases by H.A. Kramers [75]. Since fission fragments have such short range in matter, the expected distance travelled in the material, $\langle x\rangle$, is very small and so the value of $\rho_{I}$ is large based on equation 4.15. The expected range is similarly higher for relativistic electrons than for $\alpha$-particles.

This is important because a single site event with two electrons would be expected to have a greater ionization density than a single electron event of the same energy. This is based on the idea that lower energy electrons have a shorter range in a material than higher energy electrons. If the differences are significant enough, then measurements of $a_{f}$ and $a_{s}$ would allow for the rejection of singlesite events based on the number of electrons produced. A significant result here would be a step closer to the goal of reducing the background to just $2 \nu \beta \beta$. The investigation is then whether or not this goal can be achieved with the use of a two-phase time projection chamber (TPC), the mechanics and design of which are the subject of the final section of this chapter.

\subsection{Two Phase Detectors}

A two-phase detector is any detector that uses a noble gas in a combination of two phases. Of most relevance to high energy physics are detectors using combined liquid and gas phases. These detectors are most often used in low background physics. The different modes of signal generation in the liquid and gas phases can be used together to reduce backgrounds. The discussion here is based on discussions within the author's experimental group. For more detailed information, readers are encouraged to read $[62,64,76]$.

It has already been discussed in this chapter what scintillation light is and how it can be used to get information about an event. In order to understand the role of a two phase detector, the process of electroluminescence must be understood. It is actually a considerably more simple effect than scintillation, which has multiples processes contributing to the signal.

In order to take advantage of electroluminescence, there first needs to be a large electric field present. As with scintillation, a particle enters the material, in this case the material is by definition in gas phase. Again, like with the scintillator, the particle causes atoms to become ionized or excited. However, with the large electric field present, the electrons now gain kinetic energy. Above a threshold

\footnotetext{
${ }^{1}$ The primary resource is in German and is included as a citation for its historical significance rather than direct consultation in the writing of this thesis.
} 
field strength, the electrons gain enough energy to produce more ionizations and excited atoms, which in turn contribute more electrons. This is the so-called avalanche effect. The light produced by the de-excitation of the electron ion pairs and excited atoms produced in the gas gain is referred to as secondary scintillation or electroluminescent light.

Now the two phase detector can be discussed. The most practical two phase detector, and what is meant by two phase detector throughout this thesis is the gas-liquid scintillation counter. Structurally, it consists of a liquid noble gas TPC, with a thin layer of gas at the top of the detector. A large voltage is then placed across the active material to achieve the electric field required for electroluminescence. The structure of an event is as described in the subsequent sections. First, a particle interacts with the liquid part of the detector, producing a scintillation event as described in the previous section. Since there is a large electric field now, the electron drifts up through the material. When it reaches the gas layer it triggers an avalanche, producing more light that can be captured by readout electronics.

This type of detector has several immediate benefits. The most significant of these benefits is position resolution. Since the light from the electroluminscence is so intense, it can be resolved very clearly by a photomultiplier tube or, in the case of this experiment, the more advanced SiPM. Since the electroluminescent gain comes from the kinetic energy of the electric field, it is the same across the liquid gas interface. This means that the solid angle can be worked out by the distortion of the pulse. Once the solid angle is known, it is a simple exercise to work out the position of the electroluminescence in the plane of the liquid gas interface. The depth of the event can be measured by examining the time difference between the scintillation pulse and the electroluminescence signal, since the drift velocity of the electrons is a known quantity. In summary, a two phase detector gives some degree of the 3-d position resolution. This is useful for discriminating based on single site versus multi-site events. The position resolution can be further increased by using a multi-pixel device. 


\section{Chapter 5}

\section{Experimental Setup}

\subsection{System Overview}

The main component of the setup for this experiment is the time projection chamber (TPC). This was designed originally to be a two-phase TPC, meaning that both the liquid and gas phase of the xenon would be used for signals. However, due to problems with the high voltage and potential contamination in the xenon, the scope of the experiment was revised to focus on pulse-shape discrimination in the liquid portion of the xenon. A gas layer was still maintained, however without the electric field applied electroluminescence could not be observed. While it would have been potentially helpful for comparison as any practical experiment would run at high voltage, if it is indeed possible to perform pulse shape discrimination based on the number of electrons in an event it would be possible using only the scintillation channel. Unfortunately, losing the electroluminescence also loses the energy resolution in the TPC, though that isn't strictly a problem for the analysis presented in this thesis.

The xenon scintillator is held in the inner chamber of the detector, which is sealed off with $244 \mathrm{Nm}$ of torque on each of 16 screws. The only access once the detector is closed is through a tube that delivers newly condensed xenon directly to the bottom of the TPC so as to avoid surface ripples that can distort events, and two much wider outgoing pipes which carry boiled off gas for re-purification. Within this inner volume is also included the SiPM used for light collection. The SiPM was fabricated by Hammamatsu specifically for use in low temperature experiments, and has a peak sensitivity near the xenon scintillation wavelength. The electronics for extracting the signal from the SiPM was also included in this region. In order to distribute the electric field evenly through the drift (liquid) region were 12 copper rings each connected by a $12 \mathrm{M} \Omega$ resistor, to give a total resistance of $144 \mathrm{M} \Omega$. At the top grid is a field shaping mesh to eliminate fringe 
effects. Originally,the bottom grid was to hold $10 \mathrm{kV}$ and the upper grid was to hold $8 \mathrm{kV}$ to give the drift field. The electroluminescent field was to be generated by the $8 \mathrm{kV}$ to ground across the gas region. Finally the inner volume had a long parallel plate capacitor encased in glass with five spacers. The purpose of this was to measure the level of the liquid building up in the detector as the total capacitance would vary due to the dielectric constant of the condensed xenon. A diagram of the innermost part of the detector is shown in figure 5.1.

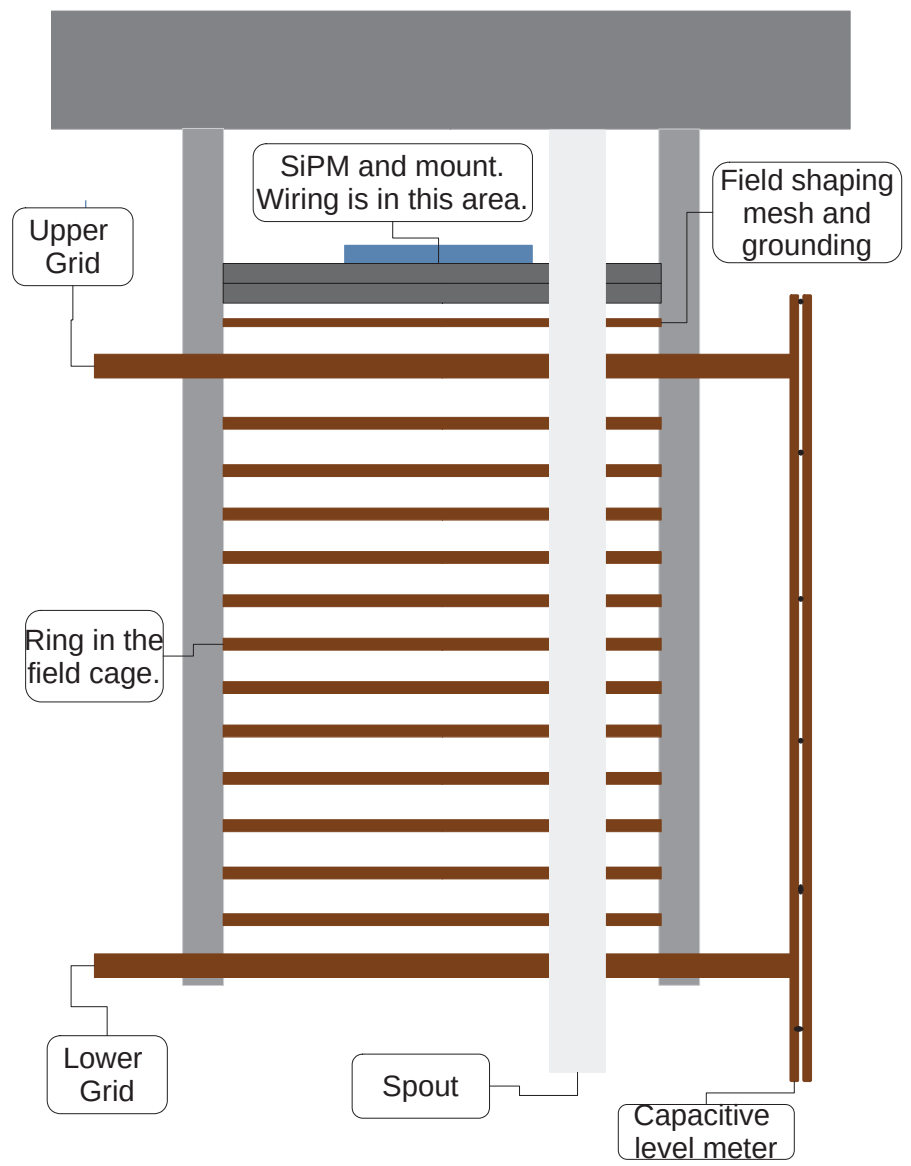

Figure 5.1: A rough diagram showing a cutaway of the inner detector. Featured in this diagram are the field cage, SiPM, spout, level probe, and grounding point. The purpose of the field cage was to establish a uniform electric field throughout the drift (liquid) region. Another copper ring is grounded to provide an even more intense electric field in the electroluminescence (gas) region. The spout, which is intended to take liquid directly to the bottom of the chamber, and the capacitive device used to measure liquid level are also shown.

In addition to figure 5.1, there are also a few photographs of the inner detector 
space. Several images were taken prior to a presentation for the Ottawa Carleton Institute for Physics. These images were taken by Callan Jessiman, the other graduate student working on this experiment. A side view of the exposed inner detector is shown in figure 5.2 and bottom-up view with part of the inner can attached to give a view of the SiPM in figure 5.3.

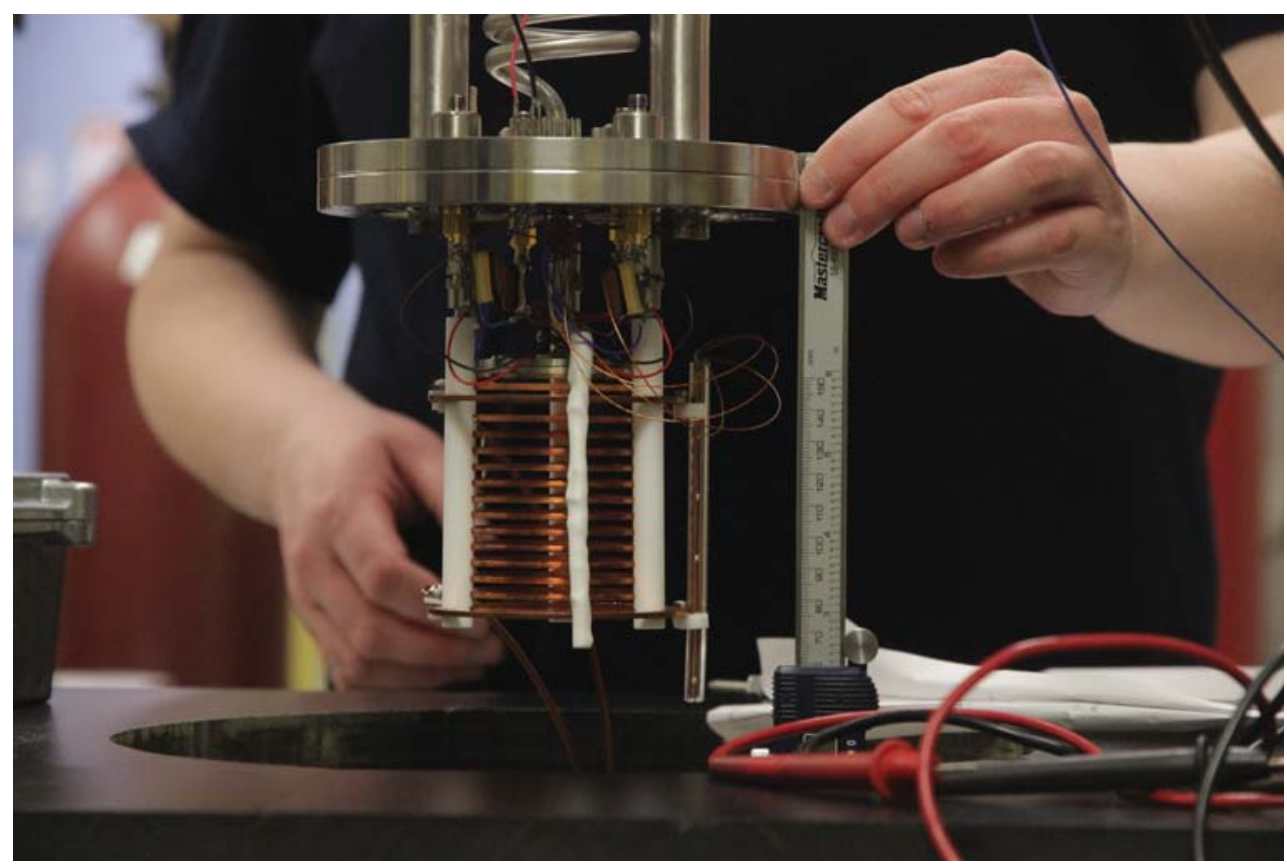

Figure 5.2: A side view of the inner chamber of the TPC. In the centre are the copper rings, with the white tubing that leads the xenon to the bottom of the detector. The purpose of the tubing is to limit ripples at the surface where they might distort the light signal. At the right of the image, alongside the ruler held up to give a sense of scale, is the capacitance probe. The broader copper rings that appear outside of the stack of twelve connected rings are the grids. The top copper ring is connected to ground, which is what would have generated the high electric field in the electroluminescent detector. Care had to be taken to fill to the top grid. This photo was taken by Callan Jessiman, used with permission.

Once sealed off there is some instrumentation connected to the outside of the inner can. Most notably there is a nichrome heater wrapped around the center of the outer can that provides approximately $32 \mathrm{~W}$ of heating when connected to 110 V AC. The purpose of the heater was to boil off gas so that the pressure inside the system remains at atmospheric pressure. It was also important to continually boil off and recirculate gas so as to purify it. The purification stages included both a MonoTorr SAES hot getter as well as a MicroTorr MC200-902FV cold getter. All gas purification apparatus were manufactured by SAES group, who are based in Milan, Italy. The intermediate space also included a 26 pin connector to read out signals from the inner can. SiPM signals were fed through SubMiniature version 


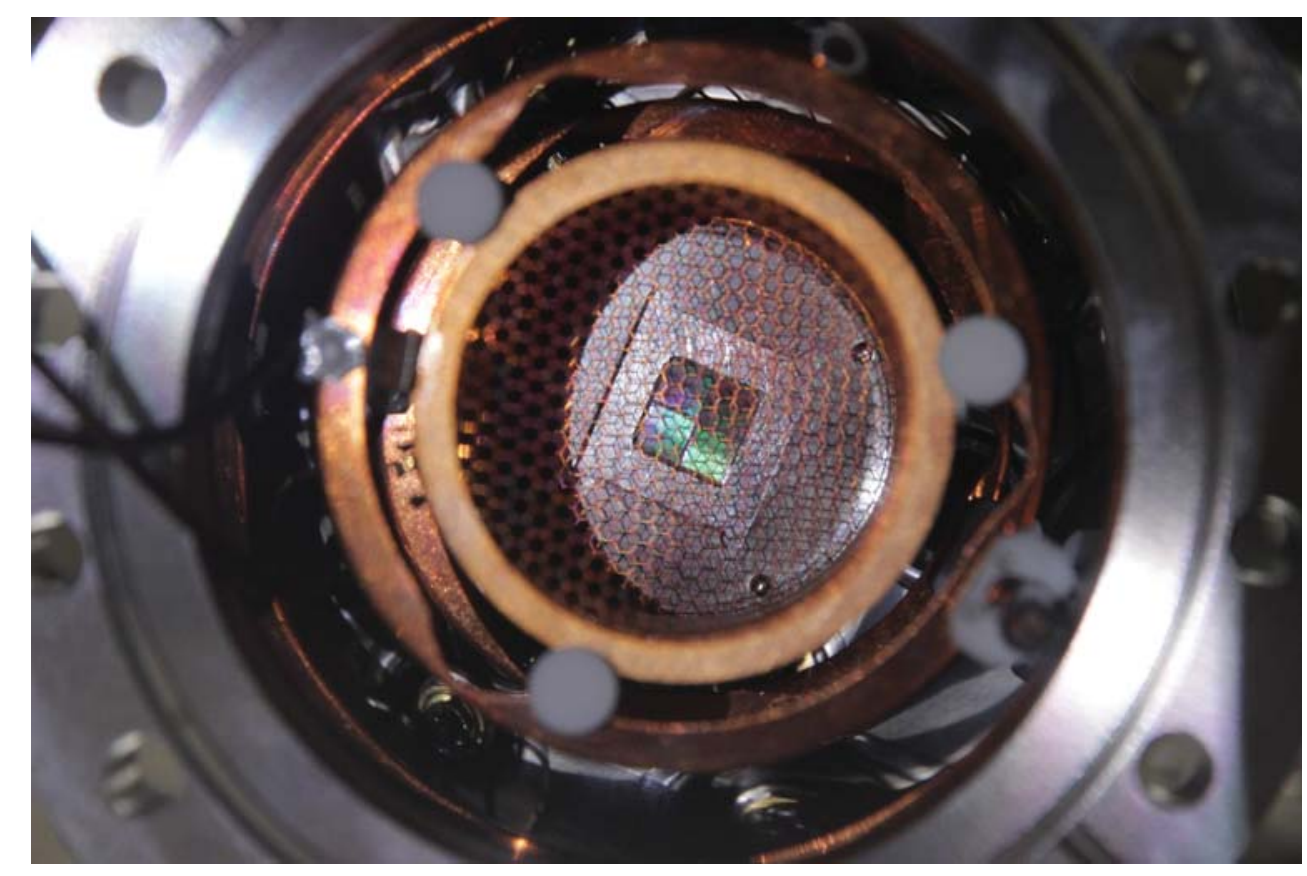

Figure 5.3: A view from underneath the inner chamber. The bottom plate to which the high voltage connections are made was not connected yet allowing for a view of the SiPM. All four pixels of the 4-channel device are visible in the photo as well as the field shaping mesh. This photo was taken by Callan Jessiman, used with permission.

A (SMA) connectors. Two platinum resistor temperature detector (RTD) were mounted either above or below the heater. The cold head of a free-piston Stirling engine (FPSE) cryo-cooler was mounted in this region as well and provided the cooling to the xenon. Once the outer can was placed around the inner can and intermediate space the system was pumped down to a high vacuum of about $10^{-7}$ mbar. The main vacuum is a T-station turbo pump manufactured by BOC Edwards who are based out of Burgess Hill, United Kingdom.

When not in use, the xenon is held in a 300 litre storage tank. The tank was filled to a pressure of $179 \mathrm{kPa}$, so that there was roughly the equivalent of 540 litres at standard pressure of high purity xenon. The TPC was filled by using a KNF pump to fill once the system pressure started to drop during condensation. KNF Neuberger is based in Trenton, New Jersey. Filling to correct height and stabilizing is discussed more in the following section.

\subsection{Temperature and Pressure Controls}

There are four temperature readouts in the system. Two of these are platinum $100 \Omega 4$-wire RTDs manufactured by LakeShore Cryotronics, who are based out 
of Westerville, Ohio. These are placed directly on the inner can either above or below the heating element. Both are connected to Omega CN8PT-220 process controllers. Omega Engineering is based out of Norwalk, Connecticut. The controller corresponding to the top RTD is set for heating action and was used to bakeout the system while the bottom RTD is read only. The bakeout was done by setting the appropriate platinum series (CN8PT) controller PID. Bakeout is an important procedure for any high purity system. During a bakeout, the heat in the system is raised while pumping under vacuum. The increase in heat causes contaminants trapped in the piping to come free. An example of a possible contaminant is water vapour. More important contaminants are those that contribute to radiopurity. The system was heated to $60{ }^{\circ} \mathrm{C}$, while a vacuum was applied to the piping, and left for three days. The outcome for this is a tighter vacuum and a reduction in airborne contaminants contributing to radiopurity.

Once the system was baked out, it was filled with Xenon gas. The first step was to fill the ballast tank to a volume of 540 litres at standard pressure. This worked out to about $179 \mathrm{kPa}$ at the tank's nominal 300 litre capacity. The reason it was overfilled was to ensure that there was enough in the detector to completely fill the inner can. From the ballast tank, the gas was then introduced into the system.

Once the gas is in the plumbing and detector with the connection open to the tank, the system can be cooled down. This was done using the FPSE cryo-cooler, a CryoTel GT series with an active vibration control (AVC) unit. The CryoTel line of cooling units are manufactured by Sunpower Inc, who are based out of Athens, Ohio. A platinum 4-wire RTD, again manufactured by LakeShore, is included with the cryo-cooler. This RTD is mounted on the cold-head of the cooler and is used by the CryoTel unit to control for target temperature. The cooler also has an internal temperature probe that is mounted within heat rejection site. Heat rejection is provided by a water-jacket. It is important to monitor this temperature in order to prevent overheating. A diagram of the cryo-cooler is shown in figure 5.4 . 


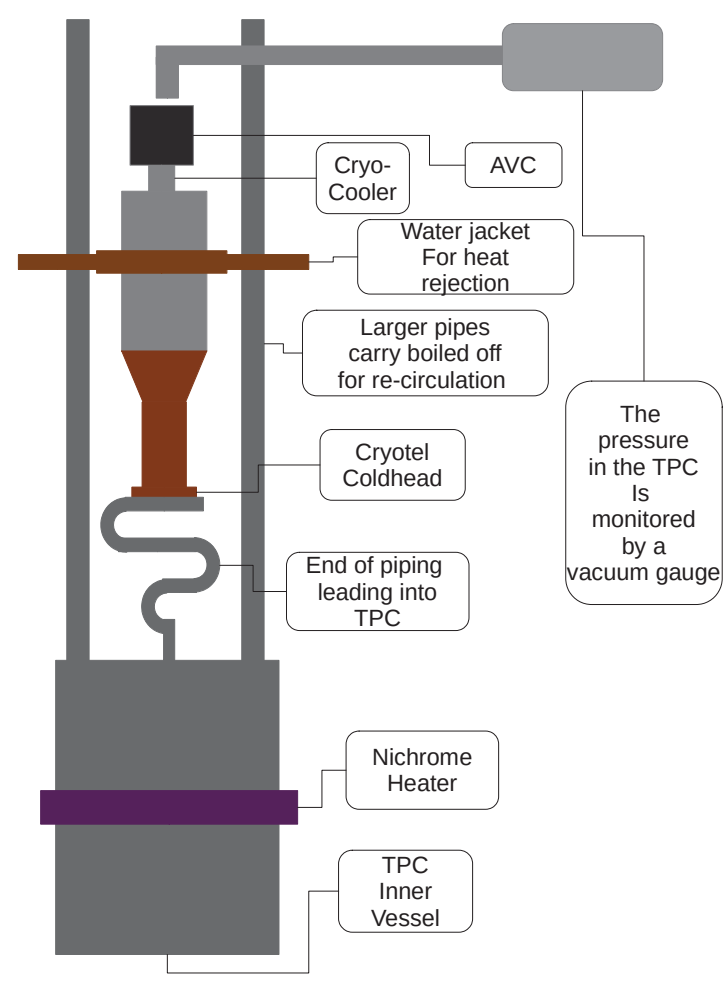

Figure 5.4: A rough schematic of the intended to show the installation of the cryo-cooler. Featured are the cooler, AVC unit, water-jacket, and heater, as well as parts of the system structure.

As the gas is cooled it is condensed at the coldhead of the cryo-cooler. Once the system becomes cool enough liquid will form. The liquid is carried by a spout ${ }^{1}$ to the bottom of the inner chamber of the detector in order to avoid forming ripples on the surface which can distort signals ${ }^{2}$. The cooler will hold the system at the boiling point of the gas as it is condensing. Once condensation starts the entire can has to come down to the boiling point in order to actually hold liquid. Eventually the pressure begins to drop. After the pressure has dropped, a KNF vacuum diaphragm pump has to be turned on in order to maintain the pressure needed to condense.

Once liquid level actually starts to build, it can be monitored by examining the capacitance between the grids. The idea is that as liquid starts to fill up the capacitance between the grids changes based on the dielectric constant of the liquid medium. There is also a designated level probe which operates in a similar manner, in this case a vertically aligned parallel plate capacitor ${ }^{3}$. Again, the

\footnotetext{
${ }^{1}$ see figure 5.1 and figure 5.2 .

${ }^{2}$ As described in the previous section

${ }^{3}$ Again, see figures 5.1 and 5.2.
} 
principle is that the capacitance between these plates changes as the liquid level fills. This second probe is measured by a four-wire capacitance probe. Ideally, both the grid capacitance and the level probe capacitance are used to establish confidence in the level. For the scintillation only run, only a ballpark of the TPC level was needed. When trying to set-up the high voltage run the level probe was used to fill and grid capacitance was used to determine when to stop. Both capacitances were measured using the same IET 1920 precision LCR meter, made by IET Labs. IET Labs is based out of Roslyn Heights, New York.

Like the two RTDs on the inner can, both of the two pressure units in this system are monitored by CN8PT-220 units from Omega Engineering. One of these pressure sensors is a 4-20 mA vacuum gauge which corresponds to 100 Psi absolute $(689.5 \mathrm{kPa})$ full scale at $20 \mathrm{~mA}$. In other words $4 \mathrm{~mA}$ corresponds to a vacuum reading and $20 \mathrm{~mA}$ corresponds to the full scale reading. The second pressure sensor, which is placed within the gas circulation piping, is a $0-10 \mathrm{~V}$ vacuum gauge capacitance manometer used to control the boil off of the two phase detector. For this device, $0 \mathrm{~V}$ corresponds to vacuum while $10 \mathrm{~V}$ corresponds to 1000 Torr $(133.3 \mathrm{kPa})$. Testing the device found that it could report a pressure up to $1106 \operatorname{Torr}(147.5 \mathrm{kPa})$. While the xenon is condensed in the TPC, the platinum series unit that reads the $0-10 \mathrm{~V}$ vacuum sensor controls the heating element based on the vapour pressure. Both devices were manufactured by Brooks instrument, based out of Hatfield, Pennsylvania.

Mounted on the outside of the can is a nichrome resistive heater. The resistance on the heater is about $390 \Omega$, and is powered by $110 \mathrm{~V}$ AC. This gives the heating power as about $31 \mathrm{~W}$. Comparatively, the cooler operating at minimum power has about $40 \mathrm{~W}$ of lift. The rest of the heating is achieved through thermal losses in the system, which are inevitable. The heater is controlled by the Omega CN8PT220 described previously. Once the pressure and temperature started dropping significantly the temperature had to be monitored carefully to avoid over-cooling and potential freezing, which happened a couple of times in early runs. When the system is under atmospheric pressure a solid state relay (SSR) switches on and current is supplied to the heater. Once gas starts to boil off, the pressure rises. The PID loop of the Omega controller helps to hold the system at constant pressure. A built in auto-tune function was used to find the PID gains for stable operation. The auto-tune typically must be run again after 24 hours. For the scintillation run, the system ran stably after the second auto-tune. A block diagram of the heating/cooling scheme is show in figure 5.5. 


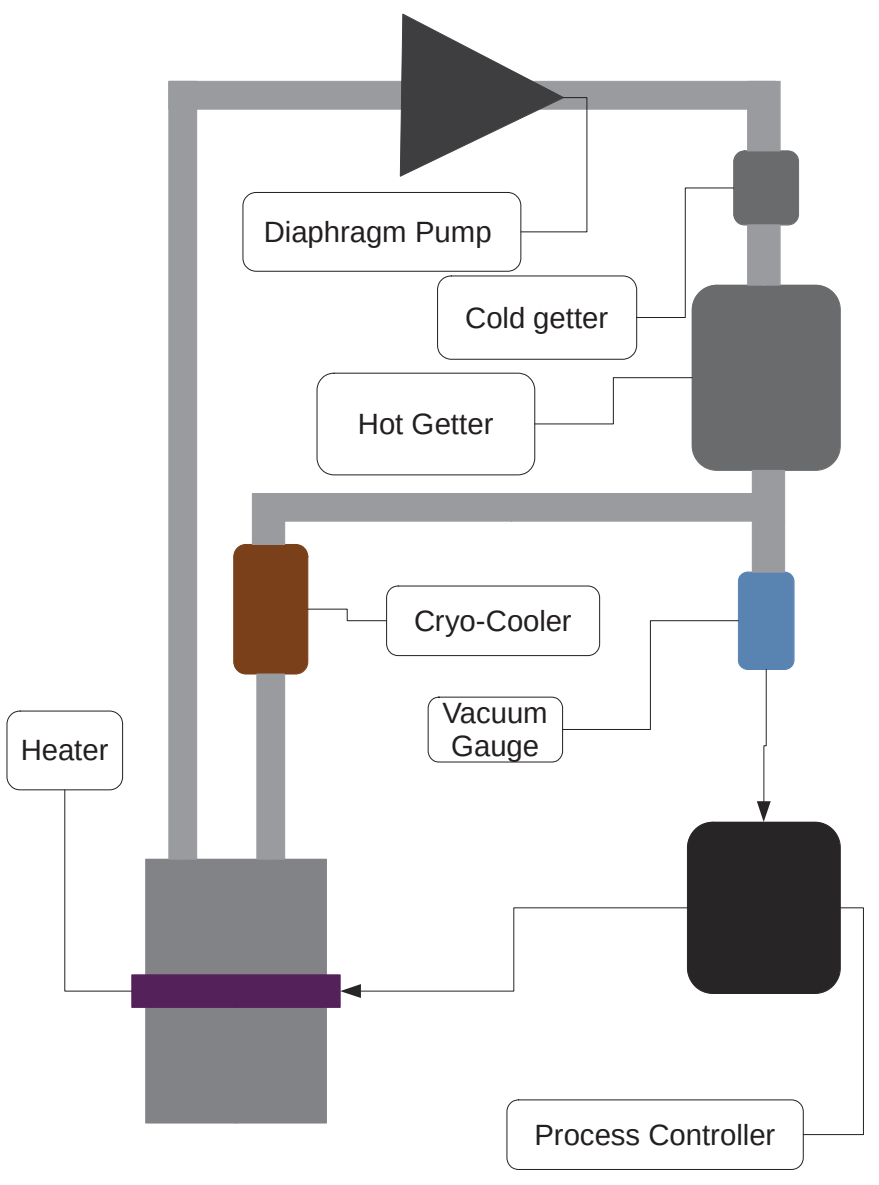

Figure 5.5: A block diagram showing the control scheme for the boil off and recirculation of xenon. The heater boils off xenon which is then recirculated using the diaphragm pump. In the recirculation route are two purifying stages. The heating/boiling is controlled by a PID unit based on feedback from a pressure gauge in the system. The cryo-cooler is operated at a constant $70 \mathrm{~W}$ power, at which it provides a lift of $40 \mathrm{~W}$.

\subsection{High Voltage Control and Procedures}

The high voltage is controlled by two OMEGA CN8PT-330-006 controllers. The 330 indicates that the devices use single pole double throw (SPDT) mechanical relays for internal connections, while the 006 indicates that they also have an isolated analog output. Each of these controllers is used to direct an UltraVolt DC voltage amplifier manufacture by Advanced Energy, who are based out of Ronkonkoma, New York. The nominal output of these devices is $20 \mathrm{kV}$. The HV supplies are also what is called inverse DC modules, which means that a $5 \mathrm{~V}$ 
control input corresponds to $0 \mathrm{~V}$ output while an input of $0 \mathrm{~V}$ corresponds to the full $20 \mathrm{kV}$ output.

The HV each have four pins that are run through the corresponding controller. These pins are: the control voltage, a $12 \mathrm{~V}$ enable pin, ground and a return pin. The control pin simply controls the output of the supply as described above, while the $12 \mathrm{~V}$ tells the device to switch on or off. Finally, there is an output pin corresponds to the output of the HV supply. This output is a standard 0-10 V, where $0 \mathrm{~V}$ corresponds to $0 \mathrm{~V}$ out and $10 \mathrm{~V}$ to the full $20 \mathrm{kV}$. After measuring this for one device, it would appear that the accuracy drifts a bit at higher voltage (i.e., the relationship between the return voltage and actual output is not exactly linear). The device is powered by an external $24 \mathrm{~V}$ volt supply. In the experience of the author, the output pin signal was never reliable. Instead, each of the high voltage modules was calibrated up to about $6 \mathrm{kV}$ and the line of best fit plotted in Excel. This was used as a significantly more accurate estimate of the voltage output.

The controllers are completely controlled within a LabVIEW application ${ }^{4}$. In this program, there are a few key features. First of all, there is the enable function. With the $12 \mathrm{~V}$ supply powered on, the enable button engages the high voltage by causing the mechanical relay to switch. This is achieved by having the program write either a designated "high" or designated "low" value to the controller setpoint. These setpoints are chosen to be out of the range of the device. This causes the relay to throw and supply the high voltage module with the $12 \mathrm{~V}$.

For safety reasons, the controller is set to always output $5 \mathrm{~V}$ when idle, ensuring that the high voltage does not develop until actively engaged by the user. Once engaged, the high voltage can be controlled by the change setpoint button in the LabVIEW front panel. When pressed, this button runs a ramp script which incrementally brings the control voltage down until the control voltage is within a certain range. First a ramp indicator lights up. The Omegas are calibrated so that the read value for a given return voltage reflects the actual high voltage output of the module it is controlling. When the setpoint is reached within one increment, the ramp indicator turns off and other commands can again be written to the controller. The increment is a set parameter that if in units of percentage of the maximum output voltage. The default is 50 , corresponding to one half of the controller's standard $10 \mathrm{~V}$ output. It should be noted that the control scheme using the UltraVolt modules never made it into the final version of the experiment. In fact, the integration of the high voltage could not be made in the timespan of

\footnotetext{
${ }^{4}$ The LabVIEW software is released by National Instruments, who have headquarters in Austin, Texas. The 2015 version of the software.
} 
this project.

\subsection{Simulating one and Two Electron Events}

In order to compare if there was a difference between one and two electron events in liquid xenon, a test had to be set up that could simulate each scenario. One electron events were simulated using a thorium-228 source. The primary gamma from this is at $2614 \mathrm{keV}$ so that when it is placed at an appropriate angle, approximately $46^{\circ}$ off from a forward detector, the Compton scattered gamma ray is at $1022 \mathrm{keV}$. The scattered electron is then $1592 \mathrm{keV}$. This can be measured using a scheme as shown in figure 5.6.

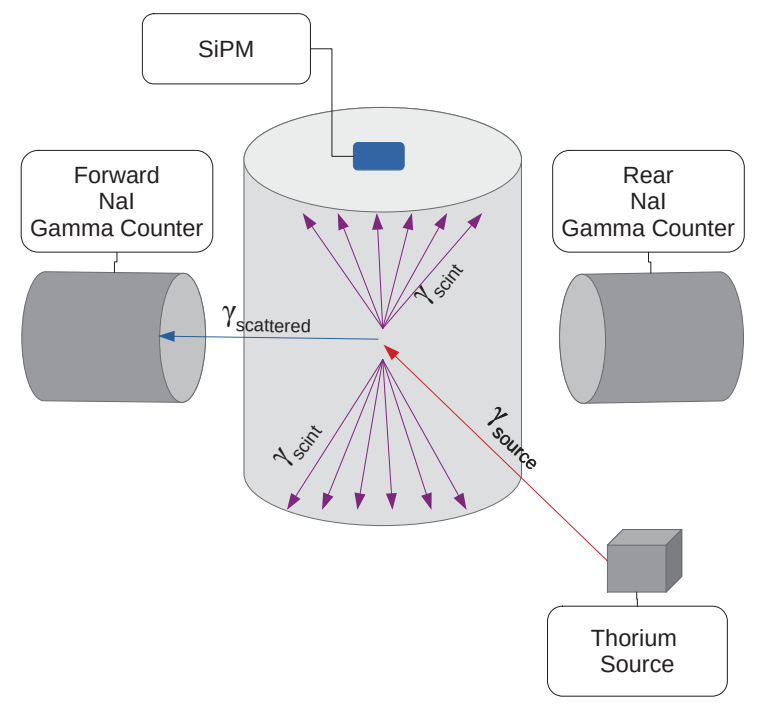

Figure 5.6: A thorium gamma ray enters the TPC and is Compton scattered onto the forward gamma counter. The angle was chosen so that the energy of this scattered gamma is $1022 \mathrm{keV}$. Scintillation light is then picked up by the SiPM.

Thorium gammas are also high enough energy that they have a substantial probability of pair producing electron-positron pairs. The gamma will pairproduce in the field of a xenon nucleus, losing $1022 \mathrm{keV}$ (or twice the electron rest mass). The remaining energy is then split between the electron and the positron. Eventually, as the positron finished its scattering, it will annihilate. This annihi- 
lation then produces back-to-back $511 \mathrm{keV}$ photons. Some of these photons produced will line up with the forward detector, such that a second gamma counter can be placed rotated $180^{\circ}$ from the first. Pair-production events can then be tagged based on coincidence in the two gamma counters, albeit with very low rate as only a fraction will be emitted such that they line up with the detectors. This system is illustrated roughly in figure 5.7.

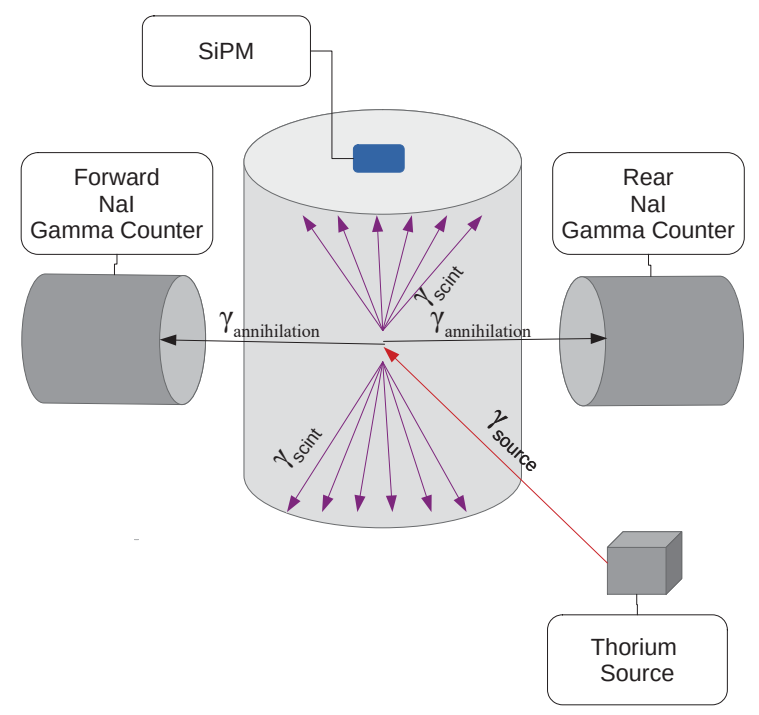

Figure 5.7: Like for the Compton scattering, pair-production is generated by interactions with a $2614 \mathrm{keV}$ thorium gamma ray. In this case, the signal is based on the annihilation of the positron that is produced in this interaction. Since these annihilations produce back-to-back gammas, some will align with both $\mathrm{NaI}$ detectors.

To collect the data, the system was set so that it triggers on coincidence between events in the TPC and in the forward detector. Specifically, it triggers when the pulse height of the sum of the SiPM channels and the forward sodium iodide detector cross a threshold level with a set time interval. Whenever a trigger occurred, the DAQ recorded for an 8 ms window separately for each of the $4 \mathrm{SiPM}$ channels and the two sodium iodide channels. There were a total of 62093 events in the main run. The following chapter discusses the sorting of these events, and presents the analysis of the timing structure of one and two electron events. In either case, the trigger for each event is coincidence between the SiPM and the 
forward $\mathrm{NaI}$ detector.

\subsection{Light Readout}

Light collection was done with a Hamamatsu S13371 multi-pixel photon counter (MPPC), which was designed for cryogenic temperatures. Hamamatsu Photonics is based out of Hamamatsu, Japan. The original idea behind using a four segment light collection device rather than something more simple like a photomultiplier tube was to potentially use it for added position resolution. It is important to note that SiPMs are a relatively new technology and there were some unknowns in their use. In two-phase operation, the light burst from electroluminescence would be distorted differently in each pixel due to differences in solid angle depending on where in the $x-y$ plane the event occurred. It was also considered a possibility that this technique could be used to add an extra layer of position resolution to the scintillatoin signal as again, some distortion would be present in different channels depending on the solid angle. Time constraints, however, prevented the author from actually investigating this, though the intensity of the peaks in the SiPM appear to have had a strong dependence on proximity to the SiPM based on the data. Another advantage of using an SiPM is that they have a very fast response time and can don't require a wavelength shifter, potentially simplifying the time characteristics as explained in the previous chapter.

The raw output from the SiPM was passed through an amplifier and into the digitizer, a CAEN 5730. The CAEN group ${ }^{5}$ is based out of Viareggio, Italy. This digitizer had a $2 \mathrm{~V}$ peak to peak full range. A former post doctoral researcher, Bjoern Lehnert, made the first version of the data acquisition (DAQ) software based on the software provided by CAEN. The other graduate student, Callan Jessiman, then made a more complete version that was used as the DAQ for the experiment. The way that the DAQworked, the digitizer was set to trigger at a certain threshold either above or below baseline. For this experiment, since the expected pulses were inverted, it was set to trigger before the baseline. The DAQ saved events as root histograms of short variables. A diagram of the trigger system is shown in figure 5.8 .

\footnotetext{
${ }^{5}$ Costruzioni Apparecchiature Elettroniche Nucleari
} 


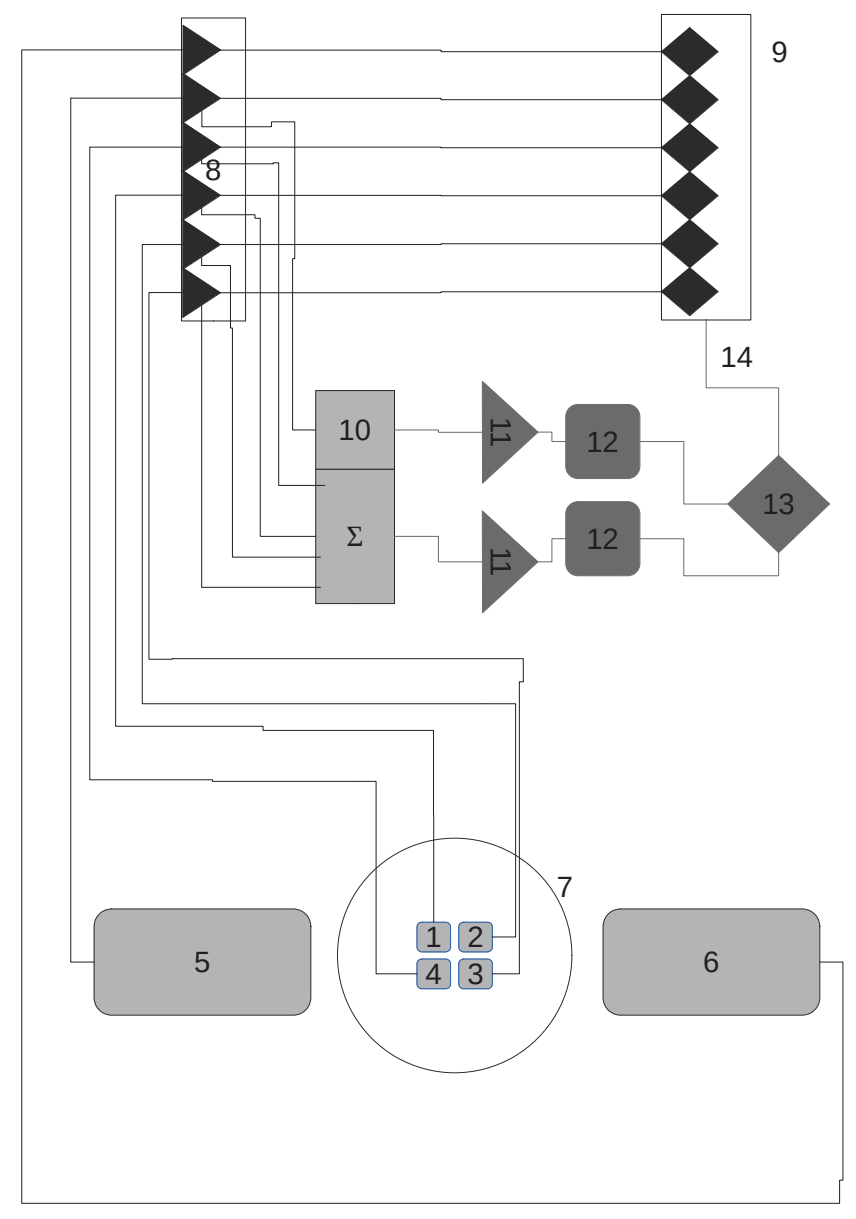

Figure 5.8: This block diagram shows the trigger system, which is explained in the rest of this section. Labels 1-4 show the four segments of the SiPM. 5 and 6 show the forward $\mathrm{NaI}$ detector and the rear detector respectively. 7 indicates the edge of the TPC, which is shown from a top view. 8 shows an amplifier unit. Each of the signals from the SiPM channels and the two gamma counters each go to their own channel on the amplifier units. 9 shows the digitizer. 10 shows a fan in/out unit which converts the forward detector and SiPM signals into logic pulses. Note that the SiPM channels are summed to form the logic pulse. 11 shows a timing filter. 12 shows a discriminator unit. 13 indicates a coincidence unit. Finally, the label 14 indicates that the coincidence signal is used as the trigger for the digitizer.

Figure 5.8 shows the trigger for events in the system. The events used in data collection are explained in the next section. Event recording is achieved based on coincidence between the forward NaI detector and the SiPM. To achieve this, all of the signals are first passed to an amplifier. The amplifier used was a LeCroy 612AM. The rear gamma counter is passed directly to the digitizer. Signals from the SiPM and the forward $\mathrm{NaI}$ detector are passed into the fan in/out unit, which 
is a LeCroy model $428 \mathrm{~F}$. Teledyne Lecroy is headquartered in Chestnut Ridge, New York. The summed SiPM and forward gamma counter signals are then passed into an Ortec 474 timing filter and a PS710 octal discriminator. Ortec and Phillips Scientific are based out of Oak Ridge, Tennessee and Mahwah, New Jersey respectively. The final stage is a C314/NL, again manufactured by Ortec, which establishes a coincidence between the SiPM and forward gamma counter. This coincidence is then used as the trigger for the DAQ system. 


\section{Chapter 6}

\section{Analysis}

\subsection{Sodium Iodide Detector Calibration}

This chapter focuses on the analysis of the data collected by the scintillation detector. The author's analysis focuses on trying to use brute-force curve fitting in order to extract the expected time constants for the fast and slow components. As discussed in the previous chapter, in order to model one and two electron events, a thorium source was used and focused on the detector. The Compton scattered events represent a single electron event at $1.59 \mathrm{MeV}$. Since thorium gammas are very high energy, they can also interact via pair production. Some pair production events will exit the detector anti-parallel with each other and can hence be used as a tag for two electron events. Furthermore, since pair production gamma's have $511 \mathrm{keV}$ energy, the total energy of this event is the same as for the single electron Compton scattering event. This is the type of analysis which could hopefully be deployed in a future xenon double-beta experiment if it could efficiently be used to tag 2-electron events, as this would reduce backgrounds around the Q-value to just the two neutrino decays. The outgoing 511 and $1022 \mathrm{keV}$ gammas are detected by two sodium-iodide detectors oriented on either side of the detector. The forward detector is placed at the Compton angle, while the rear detector is placed $180^{\circ}$ away.

Since the Compton and pair production events are not the only events that could potentially trigger the scintillation chamber in coincidence with the $\mathrm{NaI}$ crystals, for example cosmic ray muons, they had to be calibrated. To establish confidence that events are real, the gamma detectors were calibrated to the 1022 and $511 \mathrm{keV}$ energies. This was done by exposing both the forward and rear detectors to a caesium source as well to background in the room. Each of the four runs (caesium + background for each detector) was left for approximately 8000 counts. For each event, the area, length and height of the pulse were taken 
and plotted in a ROOT histogram ${ }^{1}$. Of these the pulse areas was selected as the most reliable parameter to calibrate on, due to the fact that pulse heights in the rear detector were very close to the cut-off threshold. The histograms of the pulse areas were then fit in order to find the locations of usable peaks. For the caesium runs, a Gaussian was fit to the data and the average taken to be the position of the peak corresponding to the energy of the ${ }^{137} \mathrm{Cs}$ gamma ray, which has energy $662 \mathrm{keV}$. These fits are shown for the forward detector in figure 6.1a and for the rear detector in figure $6.1 \mathrm{~b}$.

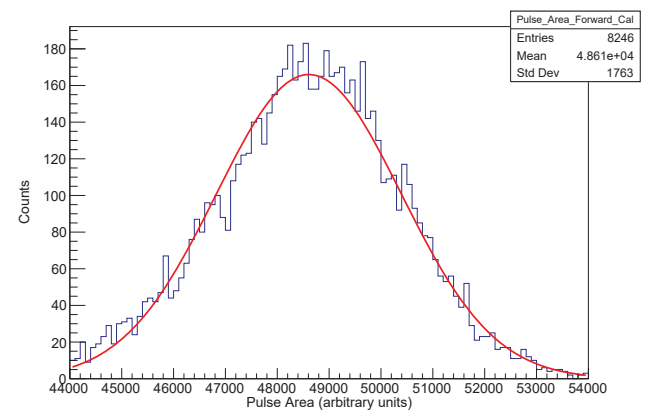

(a) Fit for the forward NaI caesium data. The $\chi^{2}$ value for the fit was 119.7 with 96 degrees of freedom. The mean was $48609 \pm 22$.

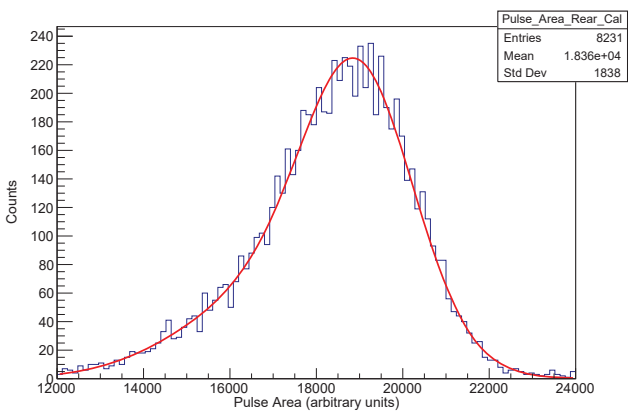

(b) Fit for the rear NaI caesium data. The $\chi^{2}$ value for the fit was 91.9 with 94 degrees of freedom. The mean was $18996 \pm 46$.

Figure 6.1: A Gaussian distribution was fitted to a histogram of pulse areas collected from sodium-iodide crystal detectors exposed to caesium-137. The mean of these pulse areas were used as calibration points in a sodium-iodide spectrum used to identify $511 \mathrm{keV}$ gamma-rays associated with pair production and 1022 $\mathrm{keV}$ gammas associated with the Compton scattering of the thorium-228 $2614 \mathrm{keV}$ gamma. The error on this peak was taken to be the error on the peak position of the fit as calculated by ROOT. The peak position and its corresponding error were used in a later linear fit calibration. For the rear detector, a double Gaussian was used as the appeared to be a shoulder effect that skewed the calibration data.

Background spectra for each of the two NaI detectors were also taken. The idea behind these spectra was to look for additional identifiable calibration points. In particular, one would expect a measurable presence of $511 \mathrm{keV}$ gammas associated with pair production and annihilation, as well as a $1460 \mathrm{keV}$ gamma signal associated with Potassium-40 which is present in concrete. Collection on these spectra was also left for about 8000 counts. Gaussian distributions were fitted onto the sub-ranges of peaks in the spectra. In both the forward and rear detector a peak corresponding to the ${ }^{40} \mathrm{~K}$ line was easily identifiable. A second peak in the

\footnotetext{
${ }^{1}$ ROOT is a data analysis framework released by CERN. CERN is based in Geneva, Switzerland.
} 
spectra was also present at lower energy. This peak was consistent with expectations for a 511 peak in the forward detector, but appeared at a higher pulse area than the 662 in the rear detector. This was likely because the threshold on the rear detector was set too high. The 511 peaks were not used for calibration of either detector. From the Gaussian sub-ranges, the peak position and error were extracted the same way as for the caesium calibration data. The background fits are shown for the forward detector in $6.2 \mathrm{a}$ and in figure $6.2 \mathrm{~b}$.

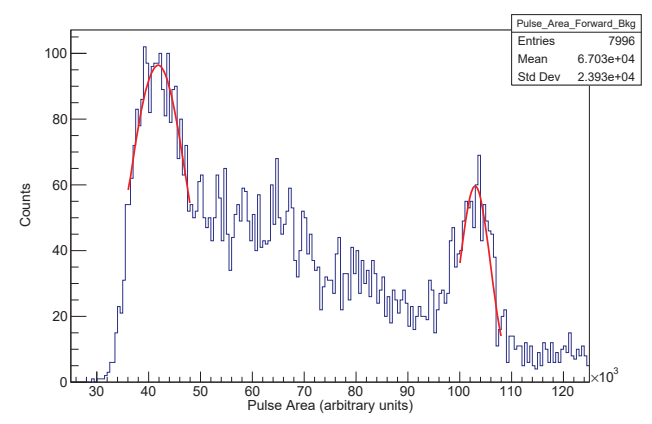

(a) Fit for the forward background spectrum data. The $\chi^{2}$ value for the ${ }^{40} \mathrm{~K}$ fit was 27.3 with 13 degrees of freedom. The mean was $102968 \pm 213$.

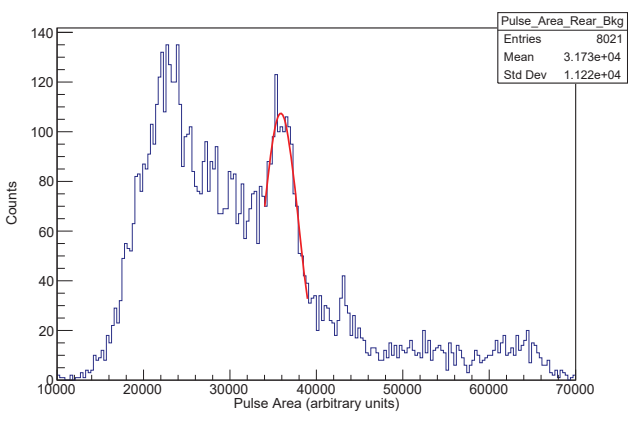

(b) Fit for the rear background spectrum data. The $\chi^{2}$ value for the ${ }^{40} \mathrm{~K}$ fit was 8.5 with 14 degrees of freedom. The mean was $35883 \pm 103$.

Figure 6.2: Gaussian peaks were fitted to the sub-ranges of the background spectra for both sodium-iodide detectors. The goal of this was to obtain extra calibration points both above and below the caesium $662 \mathrm{keV}$ line by attempting to identify the $511 \mathrm{keV}$ pair production line as well as $1460 \mathrm{keV}$ potassium-40 line. As shown, the ${ }^{40} \mathrm{~K}$ line was possible for both detectors, whereas the 511 would not have been usable for the rear detector. The pair production line was not used for calibration in either detector.

Once the peaks were found from both the background and caesium spectra for each detector, a linear fit was performed so that the data might be extrapolated to the required energies for detection of pair-production and Compton scattered events. As the signal from the $\mathrm{NaI}$ crystals is expected to be proportional to the energy of the absorbed photon [77], one would expect a linear fit of the form

$$
E=s A
$$

where $E$ is the energy of the absorbed photon, $A$ is the corresponding pulse area of the signal produced in the $\mathrm{NaI}$ crystal and $s$ is a scaling factor. In order to account for any threshold effects the fit included an intercept term. One would expect this term to be relatively small. The actual measured intercept was about $7 \%$ of the 
calibrated $662 \mathrm{keV}$ peak. In the rear detector, this number was $22 \%$. However, it is noted that the trigger threshold was set quite high for the calibration runs in the rear detector.

As will be explained in the following section, it was also important to determine the acceptable ranges on suspected Compton scattering and pair-production events in the full data runs. In order to do this, it is not enough to fit the peaks alone. The statistical spread of expected peaks must also be extrapolated. Due to the fact that $\mathrm{NaI}$ is a scintillator and thus the signal generated by the absorption of ionizing radiation is a function of the number of photons generated by that radiation, one would expected the statistical spread of an event to follow counting statistics. In other words it should be proportional to the square root of the number of photons, which is in turn proportional to the energy deposited by an ionizing event:

$$
\sigma_{E}=K \sqrt{E}
$$

where $K$ is some proportionality constant. Attempting to fit the standard deviations produced in the fitting of the gamma peaks from the calibration data yielded only a constant term. This was true even when naively attempting to fit a linear relationship to the data. In order to compensate for this, the standard deviation of a peak of pulse areas was adjusted relative to the $662 \mathrm{keV}$ Cs peak, which was most well defined, and assumed to follow the relationship

$$
\sigma_{A_{E}}=\sqrt{\frac{E}{662}} \sigma_{A_{662}}
$$

This approximation was then used to determine the ranges of expected Compton and pair production events.

\subsection{Selection of Compton Scattered Events}

Compton scattered events were important to this experiment as a tag for singleelectron events. Specifically, the forward detector was placed so as to be centred

about the expected angle for the $1022 \mathrm{keV}$ scattered photon from a ${ }^{228} \mathrm{Th}$ source. In principle, the total energies of Compton derived events at this energy would have the same total energy as two electron events generated by pair-production and annihilation. Since the NaI detector had a physical surface area facing the detector there were a range of angles accepted by the detector, and thus a range of pulse energies could be tied Compton scattering. Rather than cutting based on the Compton edge, the range was selected based upon the expected pulse areas at the $1022 \mathrm{keV}$ centre, assuming Gaussian statistics and extrapolating the error 
based on equation 6.3. The range used was based on a 2-sigma interval about the pulse area corresponding to the $1022 \mathrm{keV}$ gamma. In the arbitrary units of the measured pulse areas, the range went from 68700 to 77600 rounded off to 3 significant figures. This corresponded to a range of energies 957 to $1087 \mathrm{keV}$. While this would certainly pick up some Compton scattered gammas outside of the desired 1022 peak, these should be of high enough energy that they are still useful. A broad spectrum of events in the forward detector is shown in figure 6.3.

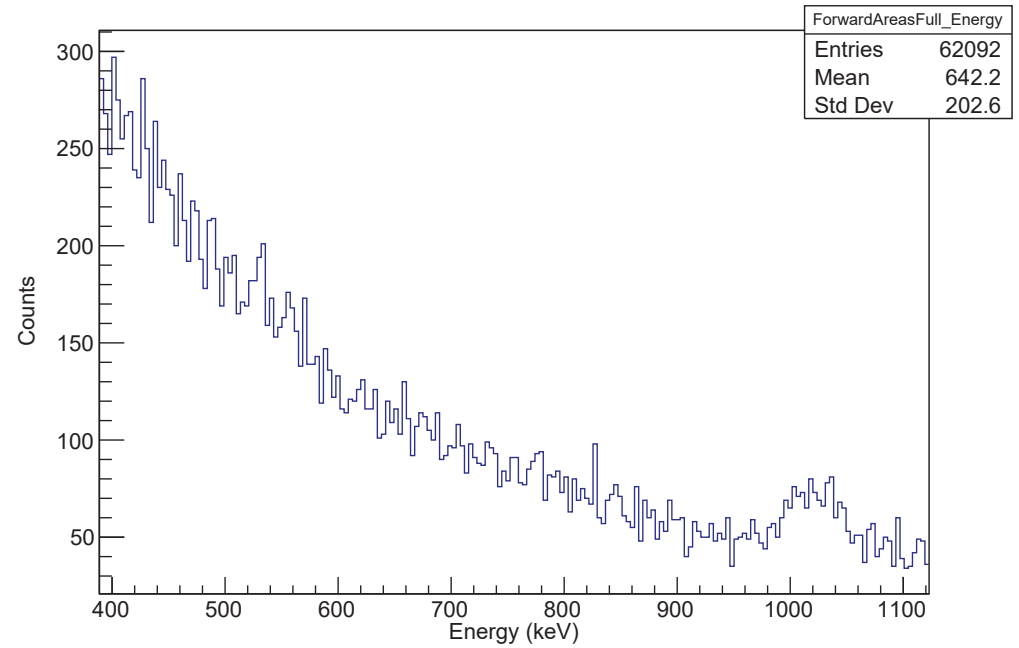

Figure 6.3: A broad spectrum of pulse-areas collected from the forward detector. This figure deliberately excludes excessively high energy events as well as low energy noise near the threshold, which dominated the spectrum. Notice that there is a discernible peak in the stated range for Compton energies. Note as well that there is no clearly identifiable peak in the lower energy range corresponding to possible pair-production events. The cuts on pair production are explained in the next section.

Making cuts based on this range of Compton energies yielded 2097 candidate events. The identifiers for these events were then saved to disk so that they could be extracted and fitted in later analysis.

\subsection{Selection of Pair-Produced Events}

The experiment was set to trigger on coincidence between the SiPM in the TPC and the forward gamma detector. Expected pair-production events are then based on a third coincidence with the rear detector. As such, the number of pair produced events is expected to be quite small in comparison to Compton or background events. This is why there is no easily discernible peak in the spectrum of 
figure 6.3, as the forward detector necessarily triggered on every event, providing a large background. The way to detect pair-production events is based on the rear detector. Figure 6.4 shows a partial spectrum of the rear detector, fit about a low energy excess.

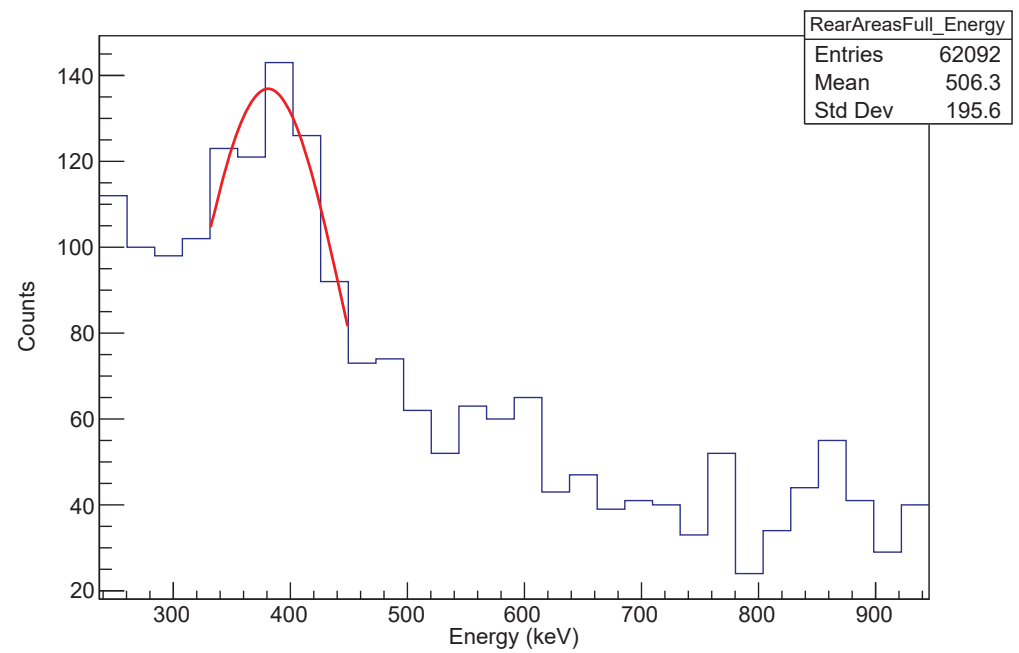

Figure 6.4: A partial spectrum of coincidence events in the rear detector. Low energy noise is again purposefully excluded from the histogram, and dominated the spectrum as in the forward detector. As in the case of calibration a Gaussian fit was performed on the sub-range of this peak. The sub-range was chosen based on the noticeable excess of events in the region, which would be expected at the pair-production energy as the detector would pick these events up from both the background and the source. Based on the energy range about the excess, it is clear that there was some inaccuracy with the caesium calibration in the rear detector.

Based on the fit shown, the energy of the excess is below what would be expected for $511 \mathrm{keV}$, however, in principle the rear detector only triggers on coincidence between both of the two NaI crystals and the TPC. As such, the only contributions to significant pulse areas in the rear detector should be a sample of the background spectrum and the $511 \mathrm{keV}$ signature line. Hence, the fit of this excess should be taken as the 511 pair production calibration point.

With this peak calibrated, information from the rear detector was used to label suspected pair production events. In order to do this, the range calculated from the fit shown in figure 6.4 was used to restrict events based on pulse area. A two sigma range was then calculated using the calibration of the forward detector to further restrict the number candidate events based on pulse area. The final result was a collection of 63 pair production events that were saved to disk for later analysis. 


\subsection{Shaping of Scintillation Traces}

This section will briefly discuss the shaping of raw traces into pulses that can be used for analysis. An example of a raw trace is shown in figure 6.5.

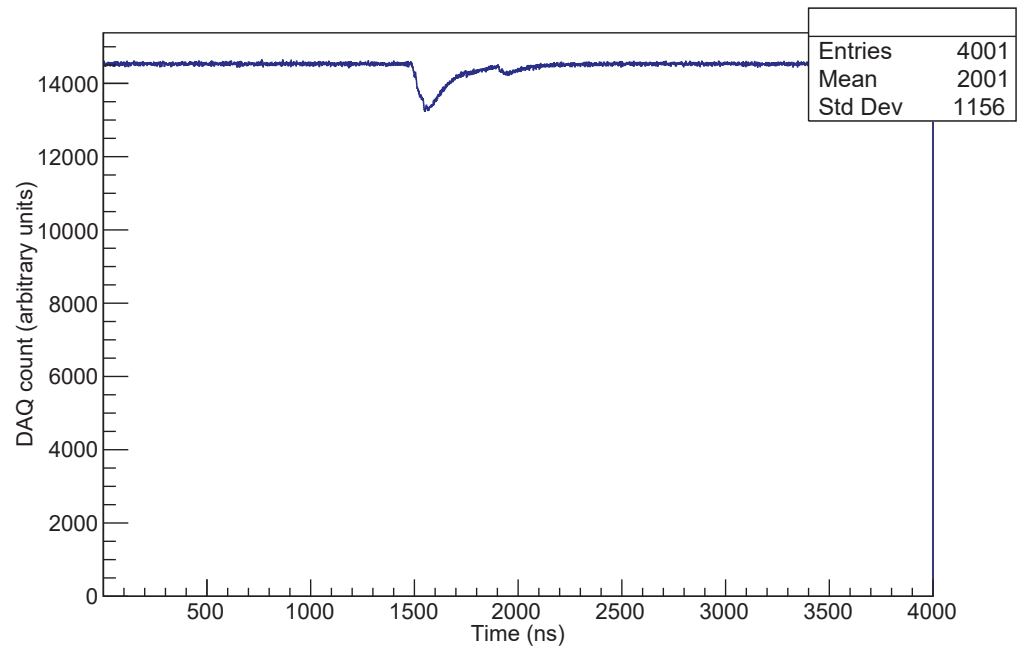

Figure 6.5: A sample TPC event. All events were collected with the same digitizer and DAQ software and so follow a similar structure. The binning is based on the time resolution of the digitizer so that each bin has a width of 2 ns, which was used to calculate the time constant later. This event is just a sample, and did not correspond to either a Compton scattered or pair-production photon.

The raw pulses can't be fit directly. In particular, the ideal pulse shape, whether it be for determining the pulse area from the detector or for fitting the pulse decay with a weighted sum of exponentials or Gale's functions is a positive pulse with zero baseline. Since the actual physical pulses are negative voltage, the baseline sits at the highest channel count. This is a particular problem for determining the uncertainties on the pulses as they are dependent on photon count and thus go as the square root of the deviation from baseline, hence the usual procedure of treating the error as the square root of the bin count is not valid.

For both the SiPM and NaI, the solution is to shape the events into a positive pulse. In the case of the NaI triggers, the pulses needed to extend from pulse start to pulse end. ROOT has built in support for finding minima and maxima of histograms. In order to estimate the pulse start and end, the procedure followed a few simple steps. First the difference between the maximum value in the histogram and the minimum value was computed, the minimum value corresponding to the maximum of the light collection pulse. Since pulses in the system corresponded to negative voltages, and due to the fact that the DAQ boxes had a fixed range 
that could be set either above or below the baseline, the maximum value in the histogram's range would be part of the baseline noise spectrum and could serve as an initial estimate of the baseline. For each bin in the histogram, iterating above and below the minimum of the trace, the term

$$
\frac{H_{b i n}-H_{\max }}{H_{\min }-H_{\max }}>0.1
$$

was evaluated. In other words, it was found where the trace fell below $10 \%$ of the difference between the maximum pulse height and the maximum, which was an initial estimate of the baseline. The main goal of estimating the pulse this way was that it reduced the number of iterations through each trace thus reducing overall computation times, which was important due to the large data set. This technique was also suitable for producing integrable pulse areas in the $\mathrm{NaI}$ as it preserves the shape of the pulse, while somewhat clipping at the bottom. In the case of the events in the TPC, these were fit based on a fixed time interval after the light peak, so pulse start and ends don't come into play except in the calculation of the baseline. This method did however produce small false pulses in traces where there was no pulse. However, this would not be a problem as these pulses had very small or negative areas that would be rejected as low energy noise.

Once the pulse start and end times were found, the baseline was estimated by averaging the value of the bins starting from time zero up until the index of the pulse start. Using the base line, the pulses were then transformed into positive pulses with baseline corrected to zero by subtracting the calculated baseline and inverting the resulting negative pulse using

$$
H_{b \rightarrow 0}=-(H-\text { baseline },
$$

where each term in equation 6.5 is understood to proceed bin by bin. Here, the error on the baseline is assumed to be the standard deviation of the mean of the baseline

$$
\sigma_{b}=\frac{s_{\text {baseline }}}{\sqrt{N_{b}}}
$$

where $s$ is the sample mean and $N_{b}$ is the number of points in the range from time zero up until the start of the pulse. One can then calculate the error on the value produced by the transformation in equation 6.5 using error propagation. As stated before, the error on a given point in the histogram is a function of the number of photons, which is reflected in the deviation from baseline of the pulse. This should give

$$
\sigma_{H}=\sqrt{(b-H)}
$$


as the error on the contents of a histogram bin. Using error propagation the total variance on the baseline corrected histograms is then given by

$$
\sigma_{H_{b \rightarrow 0}}=\sqrt{b-H+\sigma_{b}^{2}}
$$

The area is primarily important for fitting the summed exponentials onto scintillation events in the TPC.

As a final comment. The pulse areas were approximated using Romberg integration, from Wolfram MathWorld[78] this can be written as

$$
\int_{x_{1}}^{x_{N}} F(x) d x=\frac{\Delta}{3}\left(F\left(x_{1}\right)+F\left(x_{N}\right)+4 \sum_{n_{\text {even }}}^{N-1} F\left(x_{n}\right)+2 \sum_{n_{\text {odd }}}^{N-2} F\left(x_{n}\right)\right)
$$

where $\Delta$ is the separation between each of the $N$ equally spaced points in the array of $x$ values. The value of $\delta$ in this case was 1 as the x-array was just the bin number. Romberg integration assumes an odd number of points. In the case where $N$ was even an additional term was added at the end that was just $\Delta \mathrm{H}_{N}$, or the area of the final bin. This algorithm was chosen as it is the simplest many point numerical integral that can be performed, and was tested on functions similar to the expected pulse of the $\mathrm{NaI}$ and found to be sufficiently accurate for the purposes of determining pulse area. The main disadvantage of both the integration method as well as the method for identifying pulse start and end was that they were sensitive to small pulse heights. Many events in the rear NaI crystal for the calibration runs had ringing, in addition to small pulse heights. This was part of the reason that the likely $511 \mathrm{keV}$ peak in the rear NaI background spectrum was deemed unreliable.

\subsection{Pulse-Shape Discrimination in Liquid Xenon Scintillation}

The motivation behind attempting to perform pulse shape discrimination in liquid xenon was to try and identify differences between one and two electron events. There are a multitude of ways that one might attempt to separate these two quantities. For example a neural network might be used to look at subtle differences in the data, which is the subject of my colleague's thesis ${ }^{2}$. For the purpose of this analysis, the idea was to attempt to discern differences between pair production and Compton scattering by producing a best fit of the decay from maximum of the light produced in an event. The advantage of using a rudimentary technique

\footnotetext{
${ }^{2}$ See Callan Jessiman's thesis.
} 
such as brute force curve fitting is that it more easily allows for some physical sanity checks. A disadvantage, especially in the case of this experiment, is that there were inherently a number of unknowns that add a degree of difficulty to this.

In principle, one would expect the decay of the light signal to follow the two term exponential decay shown in equation 4.7, which was discussed in Chapter 4. It was also discussed, again in the fourth chapter, how the Modified Gale's function has been a popular choice for fitting scintillation curves in liquid Noble Gases. An argument was presented as to why that it was not expected that this type of analysis would be necessary for the experiment described in this thesis. Specifically, it was discussed that there was not a wavelength shifting element in the light collection and that SiPMs generally have very fast response times. There was the factor of the response time of the electronics, however this only processes the signal and does not affect that light collection itself.

In order to make the fits, the starting assumption was that the light pulse itself followed a two term exponential. The fits were performed on peaks that were shaped as described in the precious section. Once events were tagged as either Compton or pair production events, the baseline correction transformation was applied to the events in each of the channels of the TPC SiPM. The signal in any single channel should be reflective of the number of photons impinging on that pixel. Event pulses were then constructed by adding all four channels together by summing starting at the peak of the trace in each pixel. The errors on each corrected trace were then added in quadrature. Pixels were only summed when the maximum occurred in coincidence with the dominant peak. A core assumption of this is that the gain on each pixel is the same.

The basis for fitting was the use of the method of least squares form combining measurements. This is done by attempting to minimize the Chi-Squared value[79] which is given by the equation

$$
\chi^{2}(f(t))=\sum_{i=1}^{N} \frac{\left(y_{i}(t)-f(t)\right)^{2}}{\sigma_{i}^{2}}
$$

where the $y_{i}$ are the measured values, $f(t)$ is the function at the same point in time and the $\sigma_{i}$ are the measured values. ROOT has several built in methods for performing this type of calculation. The one used for this analysis was the Minuit fitting tool with the MIGRAD algorithm.

It became apparent early that noise would be a problem as the pulses in the TPC were very small. This is not unexpected as events can happen anywhere in the detector volume, with the number of photons impinging on a particular pixel 
following

$$
N_{\gamma} \propto \frac{1}{r^{2}}
$$

where $r$ is the distance from the point of light emission. Part of the signal would also be lost to reflection at the gas/liquid boundary. What was unexpected was the fact that the dominant light pulse always occurred in the same channel of the SiPM. This may indicate that the gain on each pixel was not the same, potentially affecting any statistics where channels were summed. Some sample events meant to highlight the differences between events with small pulses and those with large pulses are shown in figure 6.6.

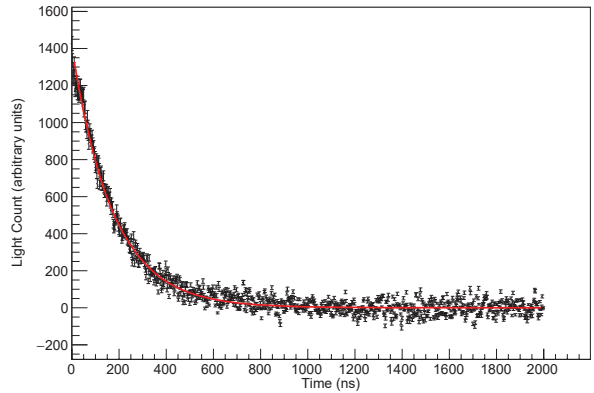

(a) A sample Compton scattered event with good signal to noise ratio.

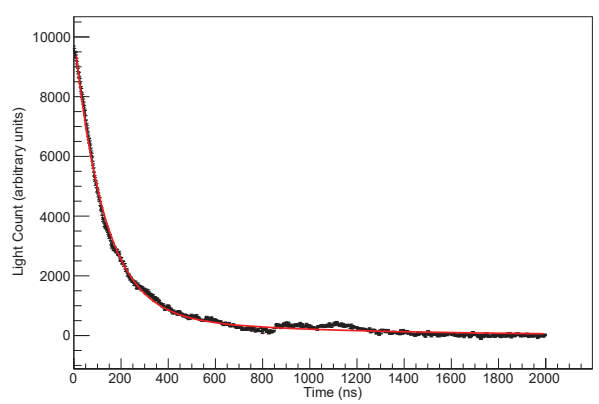

(c) A sample pair-production event with good signal to noise ratio.

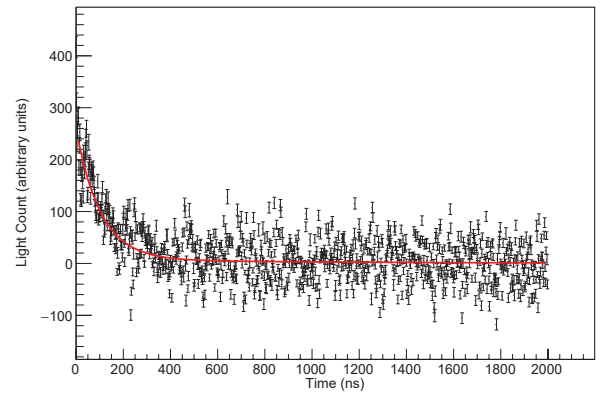

(b) A sample Compton scattered event with poor signal to noise ratio.

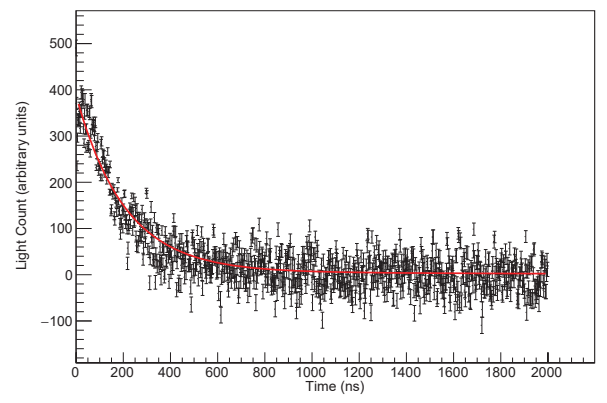

(d) A sample pair-production event with poor signal to noise ratio.

Figure 6.6: A sample of one and two electron events. The pulses were chosen to show for each of pair production and Compton scattering interactions one event with a large pulse, and thus a good signal to noise ratio, and a pulse where the summed pixels are very small and may present difficulties for the fitter. The plots presented are the final processed graphs with a superimposed two term exponential fit. The parameters of these fits were used later to attempt to quantify differences between pair production and Compton scattering.

By examining the forms of equation 6.10 it is clear that small events, which necessarily have errors that make up a large fraction of the event as they are 
derived from the square root of the light pulse in that event and the error on the baseline, are going to pose problems for a fitter. The statistics continue to get worse when pulses are added together. One may question why unsuitably small events were not rejected. The answer to this comes down to the fact that because there were already very few pair production events it would be difficult to attempt to do any analysis if these were cut.

Initially, it seemed like the simplest choice for fitting would be to take the simple two component exponential and set each of the parameters as a free parameter. Some examples of these fits were shown in figure 6.6. This would express $f(t)$ in equation 6.10 as a four parameter fit, $f\left(A_{1}, A_{2}, \tau_{1}, \tau_{2}\right)$. A layer of difficulty was added due to the fact that the two phase was never completed. Since the electroluminescence is ultimately needed to provide the energy resolution in the detector design, without this component function the TPC couldn't be properly calibrated $^{3}$. A consequence of this, among others, is that the detector response couldn't properly be taken into account. The fits describe here make the assumption that the detector left the form unchanged, but increased the time constants

$$
I(t)=a_{f} \exp \left(-\frac{t}{\tau_{f}} \prime\right)+a_{s} \exp \left(-\frac{t}{\tau_{s}} \prime\right)
$$

where the prime indicates the variable shifted by the response time. A way to test if this was indeed the case was to fit each event and produce histograms of $A_{f} / A_{s}$, $\tau_{1}$ and $\tau_{2}$ both for the set of expected Compton events as well as for the set of expected pair production events. Good fits alone are not convincing enough to accept these results, the fit parameters must make some sort of physical sense. To this end, the time constant histograms are included in figure 6.7 below and fitted with a Gaussian where statistics allowed in order to measure these time constants. 6.7a) shows the histogram of the faster time constant when the Compton scattered events are fit with a two component exponential, with the peak fit to a Gaussian giving (116.8 \pm 2.2$)$ ns. 6.7b shows the slow component from the same fit, again fit with a Gaussian, giving an average time constant of (148.1 \pm 1.6$)$ ns. The remaining two histograms show the analysis for performing the same two exponent fit on the pair-production events. $6.7 \mathrm{c}$ shows the histogram of the fast time constant from the pair-production fits. There did not appear to be enough structure in the result to perform a fit so the mean value was used and the error calculated as the standard deviation on the mean giving $(102.7 \pm 8.6)$ ns. $6.7 \mathrm{~d}$ shows the slow

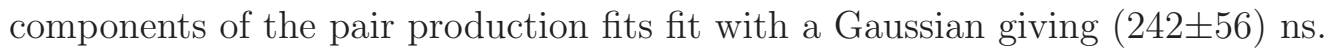

\footnotetext{
${ }^{3}$ Something that would typically be done use alphas from a Radon source.
} 


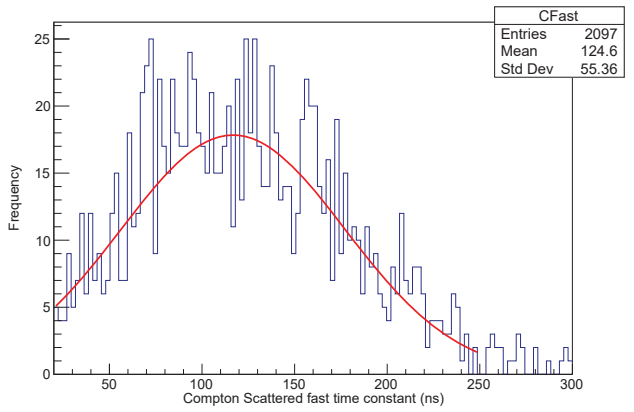

(a) Histogram of measured fast time constants in Compton Scattered events.

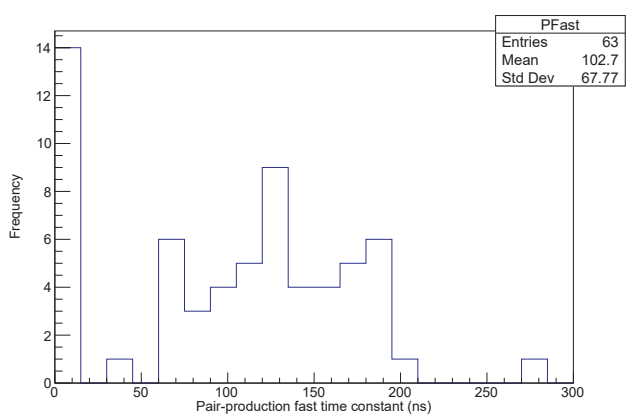

(c) Histogram of measured fast time constants in PairProduction events.

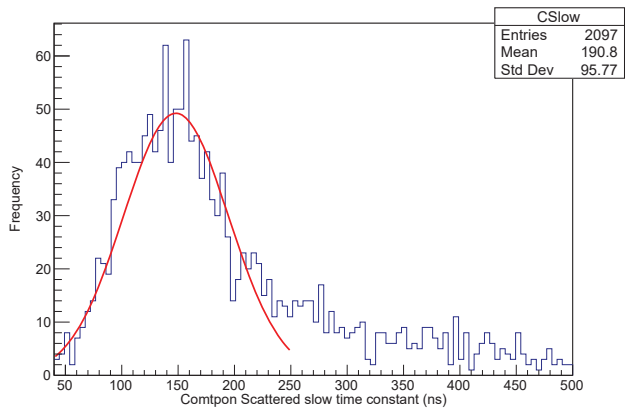

(b) Histogram of measured slow time constants in Compton Scattered events.

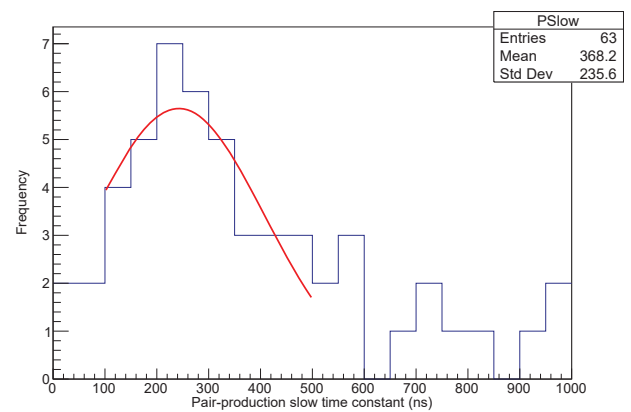

(d) Histogram of measured slow time constants in Compton Scattered events.

Figure 6.7: Histograms of the fast and slow time constants measured for pair production and Compton scattered events, fitted with a Gaussian where appropriate. This was done in order to measure the shifted fast and slow time components for each type of event.

Since the effect of the detector response on the time constants is unknown, the fitted values cannot simply be compared to the literature values. In order to evaluate whether these make physical sense, recall that the reason that two exponentials are used is to represent the two excited states that contribute to the xenon scintillation signal. Since the time constants ultimately represent a physical characteristic of xenon, one would expect statistical consistency between the $\tau_{f, c o m p t o n}$ and $\tau_{f, p p}$ as well as the corresponding slow time components. This can be done using a simple z-test statistic since Gaussian statistics are assumed based on the central limit theorem. The z-test statistic adapted to the fast component is

$$
z_{\text {fast }}=\frac{\left|\tau_{f_{\text {compton }}}-\tau_{f_{p p}}\right|}{\sqrt{\sigma_{\tau_{\text {compton }}}^{2}+\sigma_{\tau_{f p p}}^{2}}} .
$$

Setting a threshold of two sigma significance, and using the measured values pre- 
sented in figure 6.7 the values are 1.59 for $z_{\text {fast }}$ and 1.68 for $z_{\text {slow }}$. Both of these values are less than 2 and are therefore consistent at two sigma, passing the first sanity check. Another sanity check can be performed by looking at the actual quantity of interest, which is the ratio between the weighting of the fast component, $A_{f}$, and the weighting of the slow component $A_{s}$. While the goal was to look for differences in $A_{f} / A_{s}$ between the pair-production data and the Compton scattering data, there are some reasonable constraints that one should expect. One such constraint is that both weightings are expected to be positive. Another, since there are two components of scintillation light, is that excessively high values or values consistent with zero would also be an indication of non-physical behaviour. An attempt was made at binning histograms of the values of $A_{f} / A_{s}$. This was unsuccessful, however, due to the wide variation in the data. Instead, each value was recorded for each of the Compton scattering and pair-production data sets. Plots of these arrays are shown in figure 6.8.

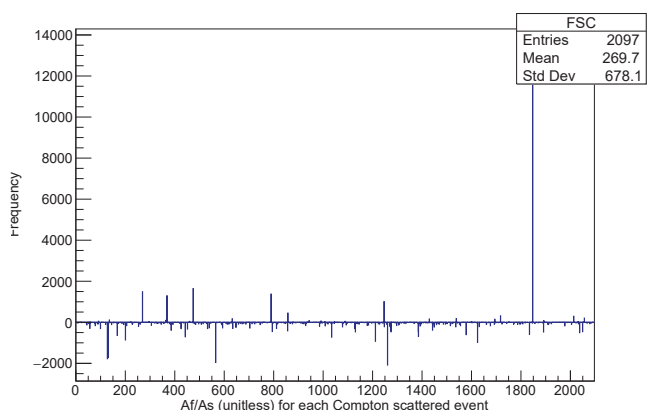

(a) The values of $A_{f} / A_{s}$ for the Compton scattering data set.

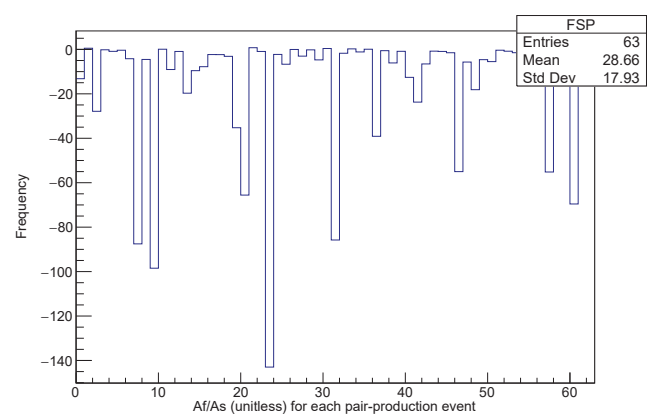

(b) The values of $A_{f} / A_{s}$ for the pair-production data set.

Figure 6.8: The measured ratios of the fast and slow weightings for both data sets. Both very clearly represent data that are not physical due to the large number of extreme and negative events.

Figure 6.8 is unfortunately where this analysis must end. The data for the measured $A_{f} / A_{s}$ clearly represents data that does not make any physical sense and cannot be used for further analysis. Either further cuts are needed or a different approach must be taken. Similarly disappointing results were observed when attempting to fit the Gale's Function and the plots are not included here. As a last attempt with the current data, the total light from each histogram was added and a final fit performed on each of these. The basis behind this idea is that it would override the noise problems in some of the smaller events without affecting the underlying distribution. Showing this using pair production as an 
example:

$$
\begin{gathered}
I_{\text {total }}=\sum_{i=1}^{N_{p p}}\left(a_{f_{p p}} \exp \left(-\frac{t}{\tau_{f}}\right)+a_{s_{p p}} \exp \left(-\frac{t}{\tau_{s}}\right)\right)_{i} \\
=\sum_{i=1}^{N_{p p}}\left(a_{f_{p p}}\right)_{i} \exp \left(-\frac{t}{\tau_{f}}\right)+\sum_{i=1}^{N_{p p}}\left(a_{s_{p p}}\right)_{i} \exp \left(-\frac{t}{\tau_{s}}\right) .
\end{gathered}
$$

Making the assumption that for each event, the measured values of the weightings sample the same underlying distribution, this reduces to

$$
I_{t o t a l}=N_{p p} a_{f_{p p}} \exp \left(-\frac{t}{\tau_{f}}\right)+N_{p p} a_{s_{p p}} \exp \left(-\frac{t}{\tau_{s}}\right)
$$

for which the ratio of the weightings is clearly preserved.

Actually fitting the summed functions proved difficult and didn't give much insight, again tending towards non-physical values for fit parameters. In addition to this, the pulses appeared to not be strictly a decaying exponential. The pulses tended to start off with a Gaussian component, which was possibly associated with detector resolution, and many pulses contained afterpulse peaks that added a new baseline offset which increased slowly. To correct for these a baseline term was added back into the fit and the pulses were cut to $800 \mathrm{~ns}$. The pulses were also plotted on log scale in the hopes that any differences might be visually identifiable. The plot for the Compton scattered data is shown in figure 6.9.

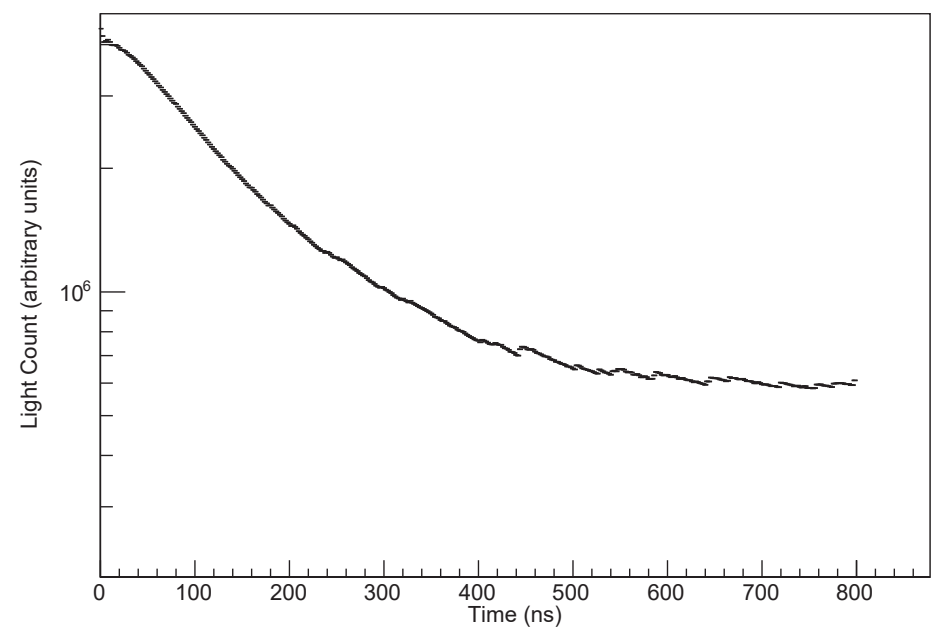

Figure 6.9: Graph showing the sum of the light pulses for the entire Compton data set.

It is not obvious just by looking at figure 6.9 if there is any easily identifiable 
trend. The best approximation might look at the area just after 200 ns where there seems to be a bump and identify this as the corner where the longer exponential starts. At this point however, the figure appears to become increasingly non-linear, and so it is not really justifiable drawing any conclusions based on this. This can be compared to the plot for the pair-production data, which is shown in figure 6.10 .

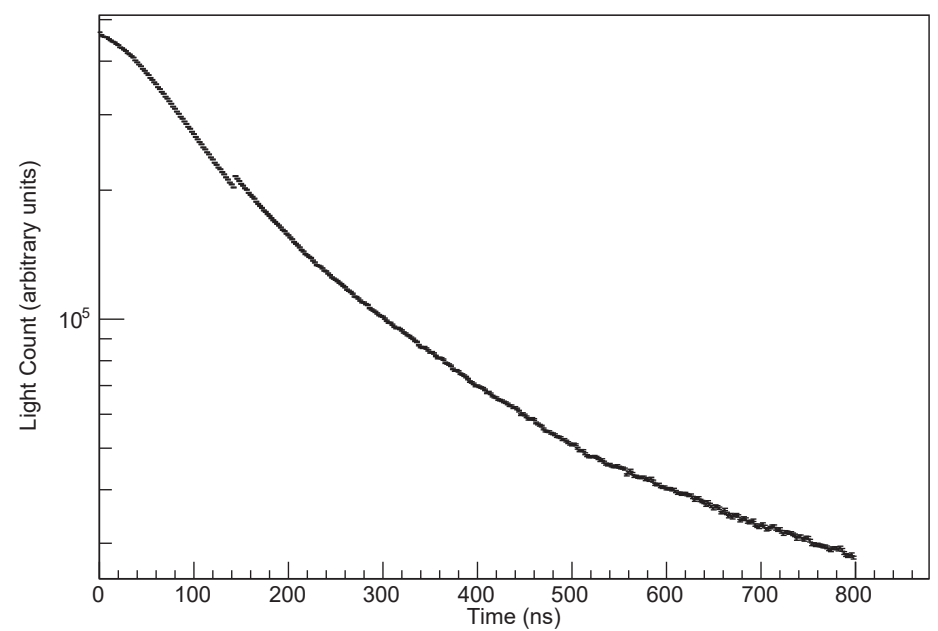

Figure 6.10: Graph showing the sum of the light pulses for the entire pairproduction data set.

Figure 6.10 has similar issues with non-linearity. In particular there is a discontinuity between 100ns and $200 \mathrm{~ns}$ that makes it extremely difficult to make any conclusions about the different decay components. Interestingly enough, if the discontinuity is ignored, figure 6.10 looks as though it may have three linear components instead of two, with the transition points located around $200 \mathrm{~ns}$, and $520 \mathrm{~ns}$ respectively. No similar visual is identifiable in figure 6.9. This could be a point of interest to explore in the future.

In both figures 6.9 and 6.10 there appear to be discontinuities in addition to the issues discussed. This is particularly noticeable around $140 \mathrm{~ns}$ in figure 6.10. It is unknown what caused these discontinuities. Since the SiPM is a novel technology, these discontinuities could be explored in future research, and may be connected to SiPM properties. At the moment, however, any comment on the source of these effects would be speculative.

Based on the analysis here, no conclusion may be made as to whether it is possible to separate one and two electron events using only pulse shape discrimination. The time constants measured in this lab are much longer than in the 
literature ${ }^{4}$, and likely reflect longer time constants in the detector electronics. It may be possible with more refined analytical techniques, but with the current limitations on time, sample size and the need for repairs on the detector it is not possible to comment on those here. The following chapter will offer a summary and attempt to outline where an experiment of the nature described in this thesis might proceed from here.

\footnotetext{
${ }^{4}$ For example see figure 4.1 in chapter 4.
} 


\section{Chapter 7}

\section{Conclusions and Future Work}

Using the data collected using the scintillation channel of the two-phase detector developed as the subject of this thesis, it was not possible to conclude that there was a difference in the ratio of singlet to triplet states occurring produced by one versus two electron events at the same energy. It was also not possible to make the conclusion that there were no differences between the two. This final chapter will briefly attempt to lay out a path forward for a xenon scintillation experiment looking to separate one and two electron events.

To start, it is prudent to consider some steps that may be taken with the detector in its current state if more time were available. The first hints as to what may be possible comes from figure 6.7 in the previous chapter, as well as from figure 6.8. Since it was determined that the time constants are at the very least consistent, this suggests that an iterative method may be employed to better refine the results. What is meant by this is that once a suitably large data set is obtained and separated into pair production and Compton scattering events, the fast and slow time components can be measured as was done in the previous chapter. The measured peaks can then be used to constrain the timing parameters and the fit can be run again, rejecting time constants that don't fall into this window. A similar method could be constructed based on the plots presented in figure 6.8, iteratively rejecting obviously non-physical values of the ratio $A_{f} / A_{s}$. The trouble with these methods comes with the phrase "suitably large". When more strict cuts are made on the data, limited data sets become a liability. In the author's earliest attempt to generate histograms of $A_{f} / A_{s}$, event rejection of the type described here was employed. Once this was done however, only two suitable pair production events remained, so effort was shifted onto finding more suitable constraints for the data that was available. Any more refined analysis technique along these lines would need more live time than what was available during this experiment.

Another possible way forward with the analysis was briefly mentioned towards the end of the previous chapter, and this was to attempt to fit more complex 
functions. This comes out of the observation from figure 6.10 that there may be three distinct components. As mentioned, this is a visual observation only and no formal attempt was made to define, or fit this. As discussed, both of the plots showing the sums of events run into the problem of detector resolution effects, anomalies (such as the discontinuity in figure 6.10) and noise, all of which can distort the plots. Like with the cutting techniques outlined previously, exploring possible extra components would require extra data sets and likely some sort of qualification of the detector response.

This leads into the big improvements that would have to be made in order to reasonably progress with this project. Most importantly, there needs to be some method of calibrating the TPC itself and there needs to be some way to ensure gas purity. In principle, properly calibrating the TPC itself involves getting twophase operation working. Actual two-phase operation would have an added level of background discrimination due to the fact that it adds some degree of position resolution. Primarily though, it would add the energy resolution that would allow the TPC to be calibrated to an alpha-decay spectrum, such as that of Radon gas. With a known alpha-spectrum, one could then de-convolve the detector output to get the detector response, which could in turn be used to further constrain fits $^{1}$. This is contingent of course on having an electroluminescence signal which requires xenon free from contamination. The group that the author was working on did attempt to integrate a high voltage system into the detector, but could not observe electroluminescence. Based on these observations, it was likely that dirty xenon was responsible for this. It is also unknown what affect electronegative impurities in the xenon would have had on timing data, but presumably there could have been an effect. Impurities have the well known effect[62] of suppressing the distance through the medium travelled by photons and drifting electrons. The key takeaway is that progressing with this experiment or a similar one would necessitate improvements in the two-phase operation and in gas purity.

For future work, the next step is to run again in scintillation mode. This should be precluded by attempting to characterize the long decay tail of the SiPM by pulsing the system with an LED and attempting to measure the response time. It may also be prudent to explore after pulsing of the SiPM. There is also the possibility that the values of the time constants may have some energy dependence, as is apparent in figure 4.1 and based on values given in [62] and [3]. Once there is an appropriate characterization of the SiPM it is suggested that an experiment be performed to attempt to measure this dependence, which could be done by varying the Compton angle. The experiment was more limited

\footnotetext{
${ }^{1}$ For example, with the detector response one could try and fit the xenon singlet and triplet lifetimes to the literature values.
} 
by pair-production events, the expected event rate for which was estimated in a simulation by Callan Jessiman. The results for this simulation are note available here. Based on the measured event rate however, which covers about 4 days worth of live-time, a similar source (on the order of $\mu \mathrm{Ci}$ ) over a month should give a few hundred pair production events.

It is the author's hope that research into the use of two-phase xenon detectors and specifically pulse shape discrimination continues. Being capable of separating one and two electron events would reduce the background for neutrino-less experiments to just the two-neutrino decays, which may be necessary to probe the full region of interest of neutrino-less double-beta decay. It is important to note that the work presented in this thesis does not rule out the possibility of separating events based on number of electrons, but instead attempts to illustrate the steps that would need to be taken towards achieving this if it is possible. 


\section{References}

[1] J. P. Ellis. TikZ-Feynman: Feynman diagrams with TikZ. Computer Physics Communications, 210:103-123, 2016. doi: doi:10.1016/j.cpc.2016. 08.019arXiv:1601.05437.

[2] B. Kayser. Are neutrinos their own antiparticles? Journal of Physics: Conference Series, 173:012013, 2009. doi: https://doi.org/10.1088\%2F1742-6596\% 2F $173 \% 2 \mathrm{~F} 1 \% 2 \mathrm{~F} 012013$.

[3] A. Hitachi, T. Takahashi, N. Funayama, K. Masuda, J. Kikuchi, and T. Doke. Effect of ionization density on the time dependence of luminescence from liquid argon and xenon. Phys. Rev. B, 27(9):5279-5285, 1983. doi: https: //doi.org/10.1103/PhysRevB.27.5279.

[4] D. Griffiths. Introduction to Elementary Particles. WILEY-VCH Verlag GmbH \& Co. KGaA, Weinheim, Baden-Württemberg, 2008.

[5] M. Peskin and D. Schroeder. An Introduction to Quantum Field Theory. Westview Press, Boulder, Colorado, 2016.

[6] A. Balantekin and B. Kayser. On the properties of neutrinos. Annu. Rev. Nuc. Par. Sci., 68:313-338, 2018. doi: https://doi.org/10.1146/ annurev-nucl-101916123044.

[7] Q. Ahmad et al. Direct evidence for neutrino flavour transformation from neutral-current interactions in the Sudbury Neutrino Observatory. Phys. Rev. Lett., 89(1):011301, 2002. doi: https://doi.org/10.1103/PhysRevLett. 89.011301.

[8] Y. Fukuda et al. Evidence for oscillation of atmospheric neutrinos. Phys. Rev. Lett., 81(8):1562-1567, 1998. doi: https://doi.org/10.1103/PhysRevLett.81. 1562 .

[9] G. Fantini, A. Gallo Rosso, F. Vissani, and V. Zema. Introduction to the formalism of neutrino oscillations: A tutorial for graduate students and young 
researchers. chapter 2, pages 37-119. World Scientific, Singapore, 2018. ISBN 978-981-322-608-1. doi: arXiv:1802.05781v1.

[10] S. Petcov. The nature of massive neutrinos. Advances in High Energy Physics, 2013:852987, 2013. doi: http://dx.doi.org/10.1155/2013/852987.

[11] S. Bilenky. Neutrino in standard model and beyond. Phys. Part. Nucl, 46: 475, 2015. doi: arXiv:1501.00232[hep-ph].

[12] P. A. R. Ade et al. Planck 2013 results. XVI. Cosmological parameters. Astron. Astrophys., 571:A16, 2014. doi: https://doi.org/10.1051/0004-6361/ 201321591.

[13] N. Aghanim et al. Planck 2018 results. VI. Cosmological parameters, 2018. URL https://arxiv.org/abs/1807.06209.

[14] S.M. Bilenky, J. Hošek, and S. T. Petcov. On the oscillations of neutrinos with Dirac and Majorana masses. Phys. Lett. B, 94:495-498, 1980. doi: https://doi.org/10.1016/0370-2693(80)90927-2.

[15] M. Kobayashi and T. Maskawa. CP-Violation in the renormalizable theory of weak interaction. Progress of Theoretical Physics, 49(2):652-657, 1973. doi: https://doi.org/10.1143/PTP.49.652.

[16] N. Cabibbo. Time reversal violation in neutrino oscillation. Phys. Lett. B, 75 (3):333-335, 1978. doi: https://doi.org/10.1016/0370-2693(78)90132-6.

[17] I. Girardi, S.T. Petcov, and A.V. Titov. Predictions for the Majorana CP violation phases in the neutrino mixing matrix and neutrinoless double beta decay. Nucl. Phys. B, 911:754-804, 2016. doi: https://doi.org/10.1016/j. nuclphysb.2016.08.019.

[18] S. Al Kharusi. nEXO pre-conceptual design report. 2018. URL arXiv: 1805.11142v2.

[19] M. Gell-Mann, P. Ramond, and R. Slansky. Complex spinors and unified theories. In Conf. Proc. Supergravity Workshop, Stony Brook, New York, 1979. doi: arXiv:1306.4669[hep-th].

[20] T. Yanagida. Horizontal symmetry and masses of neutrinos. Prog. Theor. Phys., 64(3):1103-1105, 1980. doi: https://doi.org/10.1143/PTP.64.1103.

[21] J. Schecter and J. Valle. Neutrinoless double- $\beta$ decay in $\mathrm{SU}(2) \times \mathrm{U}(1)$ theories. Phys. Rev. D., 25(11):2951-2954, 1982. doi: https://doi.org/10.1103/ PhysRevD.25.2951. 
[22] G. Benato. Effective Majorana mass and neutrinoless double beta decay. Eur. Phys. J. C., 75(11):563, 2015. doi: https://doi.org/10.1140/epjc/ s10052-015-3802-1.

[23] L. Cardani. Neutrinoless double beta decay overview. In 15th International Workshop on Tau Lepton Physics, Amsterdam, The Netherlands, September 2018. doi: arXiv:1810.12828v2[nucl-ex].

[24] M. Dolinski, A. Poon, and W. Rodejohann. Neutrinoless double-beta decay: Status and prospects. Annu. Rev. Nuc. Par. Sci., 69:1-35, 2019. doi: arXiv: 1902.04097v1[nucl-ex].

[25] J. Albert et al. Sensitivity and discovery potential of the proposed nEXO experiment to neutrinoless double- $\beta$ decay. Phys. Rev. C., 97(6):065503, 2017. doi: https://doi.org/10.1103/PhysRevC.97.065503.

[26] F. Vissani. Signal of neutrinoless double beta decay, neutrino spectrum and oscillation scenarios. JHEP, 1999(6):022-022, 1999. doi: https://doi.org/10. 1103/PhysRevD.38.3550.

[27] A. Aprahamian et al. Reaching for the horizon: The 2015 long range plan for nuclear science. Technical Report OSTI 1296778, United States Department of Energy, United States, September 2015. URL https://www.osti.gov/biblio/ 1296778-reaching-horizon-long-range-plan-nuclear-science.

[28] M. Jiang et. al. Atmospheric neutrino oscillation anlysis with improved event reconstruction in Super-KamiokandeIV. Submitted to PTEP, 2019. doi: arXiv:1901.03230[hep-ex].

[29] K. Abe et al. Measurement of neutrino and antineutrino oscillations by the T2K experiment including a new additional sample of $\nu_{e}$ interactions at the far detector. Phys. Rev. D., 96(9):092006, 2017. doi: https://doi.org/10. 1103/PhysRevD.96.092006.

[30] M.A. Acero et al. First measurement of neutrino oscillation parameters using neutrinos and antineutrino by NOvA. Phys. Rev. Lett., 123(15):151803, 2019. doi: https://doi.org/10.1103/PhysRevLett.123.151803.

[31] A. Giuliani and A. Poves. Neutrinoless double-beta decay. Advances in High Energy Physics, 2012:1-38, 2012. doi: http://dx.doi.org/10.1155/2012/ 857016 . 
[32] M. Goeppert-Mayer. Double beta-disintegration. Phys. Rev., 48(6):512-516, 1935. doi: https://doi.org/10.1103/PhysRev.48.512.

[33] S. Elliott, A. Hahn, and M. Moe. Direct evidence for two-neutrino doublebeta decay in ${ }^{82}$ Se. Phys. Rev. Lett., 59(18):2021-2023, 1987. doi: https: //doi.org/10.1103/PhysRevLett.59.2020.

[34] A. Barabash. Precise half-life values for two-neutrino double- $\beta$ decay. Phy. Rev. C, 81(3):035501, 2010. doi: https://doi.org/10.1103/PhysRevC.81. 035501.

[35] N. Ackerman et al. Observation of two-neutrino double-beta decay in ${ }^{136} \mathrm{Xe}$ with EXO-200. Phys. Rev. Lett, 107(21):212501, 2011. doi: https://doi.org/ 10.1103/PhysRevLett.107.212501.

[36] E. Aprile et al. Observation of two-neutrino double electron capture in ${ }^{124} \mathrm{Xe}$ with XENON1T. Nature, 568(7753):532-535, 2019. doi: https://doi.org/10. 1038/s41586-019-1124-4.

[37] E. Majorana. Teoria simmetrica dell'elettrone e del positrone. Il Nuovo Cimento, 14:171-184, 1937. doi: https://doi.org/10.1007/BF02961314.

[38] G. Racah. Sulla simmetria tra particelle e antiparticelle. Il Nuovo Cimento, 14:322-328, 1937. doi: https://doi.org/10.1007/BF02961321.

[39] W. Furry. On transition probabilities in double beta-disintegration. Phys. Rev., 56(12):1184-1193, 1939. doi: https://doi.org/10.1103/PhysRev.56. 1184 .

[40] K. Eguchi et al. First results from KamLAND: Evidence for reactor antineutrino disappearance. Phys. Rev. Lett., 90(2):021802, 2003. doi: https: //doi.org/10.1103/PhysRevLett.90.021802.

[41] K. McFarland. Neutrino interactions. In Neutrinos in particle physics, astrophysics and cosmology. Proceedings, 61st Scottish Universities Summer School in Physics, St. Andrews, UK, 26 June - 1 July 2006. doi: arXiv:0804.3899.

[42] C. Balázs. Baryogenesis: A small review of the big picture. In Interplay between Particle and Astroparticle Physics workshop, London, UK, 18-22 August 2014.

[43] A. Sakharov. Violation CP invariance, C asymmetry, and baryon asymmetry of the universe. ZhETF Pis'ma, 5(1):32-35, 1967. doi: https://doi.org/10. 1070\%2Fpu1991v034n05abeh002497. 
[44] M. Fukugita and T. Yanagida. Baryogenesis without grand unification. Phys. Lett. B, 174(1):45-47, 1986. doi: https://doi.org/10.1016/0370-2693(86) 91126-3.

[45] F. Avignone III, S. Elliott, and J. Engel. Double beta decay, Majorana neutrinos, and neutrino mass. Rev. Mod. Phys., 80(2):481-516, 2008. doi: https://doi.org/10.1103/RevModPhys.80.481.

[46] M. Redshaw, E. Wingfield, J. McDaniel, and E. Myers. Mass and doublebeta-decay Q value of ${ }^{136}$ Xe. Phys. Rev. Lett., 98(5):053003, 2007. doi: https: //doi.org/10.1103/PhysRevLett.98.053003.

[47] P. McCowan and R. Barber. Q value for the double- $\beta$ decay of ${ }^{136}$ Xe. Phys. Rev. C., 82(2):024603, 2010. doi: https://doi.org/10.1103/PhysRevC.82. 024603 .

[48] M. Auger et al. The EXO-200 detector, part 1: Detector design and construction. JINST, 7:P05010, 2012. doi: 10.1088/1748-0221/7/05/P05010.

[49] J. Albert et al. Search for neutrinoless double-beta decay with the upgraded EXO-200. Phys. Rev. Lett., 120(7):072701, 2018. doi: 10.1103/PhysRevLett. 120.072701 .

[50] E.-I. Esch, T. Bowles, A. Hime, A. Pichlmaier, R. Reinfarth, and H. Wollnik. The cosmic ray muon flux at WIPP. Nucl. Instrum. Meth. Phys. Res. A., 538:516-525, 2005. doi: https://doi.org/10.1016/j.nima.2004.09.005.

[51] E. Conti et al. Correlated fluctuations between luminescence and ionization in liquid xenon. Phys. Rev. B., 68(5):054201, 2008. doi: https://doi.org/10. 1103/PhysRevB.68.054201.

[52] J. Albert et al. Search for Majorana neutrinos with the first two years of EXO-200 data. Nature, 510:229-234, 2014. doi: doi:10.1038/nature13432.

[53] X. Sun et al. Study of silicon photomultiplier performance in external electric fields. JINST, 13:T09006, 2018. doi: arXiv:1807.03007v1[physics.ins-det].

[54] A. Gando et al. Search for Majorana neutrinos near the inverted mass hierarchy region with KamLAND-Zen. Phys. Rev. Lett., 117(8):109903, 2016. doi: https://doi.org/10.1103/PhysRevLett.117.082503.

[55] A. Gando et al. Measurement of the double- $\beta$ decay half-life of ${ }^{136}$ Xe with the KamLAND-Zen experiment. Phys. Rev. C., 84(4):045504, 2012. doi: https://doi.org/10.1103/PhysRevC.85.045504. 
[56] D.R. Artusa et al. Searching for neutrinoless double-beta decay of ${ }^{130} \mathrm{Te}$ with CUORE. Advances in High Energy Physics, 2015:879871, 2015. doi: http://dx.doi.org/10.1155/2015/879871.

[57] C. Alduino et al. Double-beta decay of ${ }^{130} \mathrm{Te}$ to the first $0^{+}$excited state of ${ }^{130}$ Xe with CUORE-0. Eur. Phys. J. C, 79:795, 2019. doi: https://doi.org/ 10.1140/epjc/s10052-019-7275-5.

[58] A. Caminata et al. Results from the CUORE experiment. Universe, 5(1):10, 2019. doi: https://doi.org/10.3390/universe5010010.

[59] S.I. Alvis et al. Search for neutrinoless double- $\beta$ decay in ${ }^{76} \mathrm{Ge}$ with $26 \mathrm{~kg}$ yr of exposure from the MAJORANA DEMONSTRATOR. Phys. Rev. C., 100: 025501, 2019. doi: https://doi.org/10.1103/PhysRevC.100.025501.

[60] C.E. Aalseth et al. Search for neutrinoless double- $\beta$ decay in ${ }^{76}$ Ge with 26 $\mathrm{kg}$ yr of exposure from the MAJORANA DEMONSTRATOR. Phys. Rev. Lett., 120(13):132502, 2018. doi: DOI:https://doi.org/10.1103/PhysRevLett. 120.132502 .

[61] M. Agostini et al. Probing Majorana neutrinos with double- $\beta$ decay. Science, 365:1445-1448, 2019. doi: 10.1126/science.aav8613.

[62] E. Aprile, A. Bolotnikov, A. Bolozdynya, and T. Doke. Noble Gas Detectors. WILEY-VCH Verlag GmbH \& Co. KGaA, Weinheim, Baden-Württemberg, Germany, 2006.

[63] P. Belli, R. Bernabei, S. d'Angelo, L. Andreanelli, F. Bronzini, A. Buccheri, A. Incicchitti, and D. Prosperi. Liquid-xenon detectors and their applications. Nucl. Instrum. Meth. in Phys. Res. A, 299(1):191-194, 1990. doi: https: //doi.org/10.1016/0168-9002(90)90774-Z.

[64] E. Aprile and T. Doke. Liquid xenon detectors for particle physics and astrophysics. Rev. Mod. Phys., 82(3):2053-2097, 2010. doi: 10.1103/RevModPhys. 82.2053 .

[65] S. Kubota, M. Hishida, M. Suzuki, and J. Ruan (Gen). Dynamical behavior of free electrons in the recombination process in liquid argon, krypton, and xenon. Phys. Rev. B., 20(8):3486-3496, 1979. doi: https://doi.org/10.1103/ PhysRevB.20.3486.

[66] T. Doke, A. Hitachi, J. Kikuchi, K. Masuda, H. Okada, and E. Shibamura. Absolute scintillation yields in liquid argon and xenon for various particles. 
Jpn. J. Appl. Phys., 41(3):1538-1545, 2002. doi: https://doi.org/10.1143/ JJAP.41.1538.

[67] Marlo Martin. Exciton self-trapping in rare-gas crystals. J. Chem. Phys., 54 (8):3289-3299, 1971. doi: https://doi.org/10.1063/1.1675342.

[68] T. Doke. Scintillation of liquid xenon and its application to nuclear radiation detectors. In IEEE International Conference on Dielectric Liquids, 2005. ICDL 2005., pages 293-300, Coimbra, Portugal, 26 June - 1 July 2005.

[69] A. Molchanov. Lasers in the vacuum ultraviolet and in the x-ray regions of the spectrum. Sov. Phys. Usp., 15(1):124-129, 1972. doi: https://doi.org/10. 1070/PU1972v015n01ABEH004950.

[70] S. Kubota, M. Hishida, and J. Raun. Evidence for a triplet state of the self-trapped exciton states in liquid argon, krypton and xenon. J. Phys. C: Solid State Phys., 11(12):2645-2651, 1978. doi: https://doi.org/10.1088/ 0022-3719/11/12/024.

[71] N. Gale. Lifetimes of the first excited states of $\mathrm{B}^{10}, \mathrm{O}^{17}$ and $\mathrm{F}^{17}$ (ii): Analysis of delayed coincidence lifetime measurements. Nucl. Phys., 38:252-258, 1962. doi: https://doi.org/10.1016/0029-5582(62)91032-5.

[72] D. Akimov, A. Bolzdynya, A. Burenkov, D. Churakov, T. Osipova, O. Petshina, G. Safronov, G. Smirnov, and V. Solovov. Time characteristics of scintillations excited in xenon doped liquid krypton by relativistic particles. In 1996 IEEE NSS Conf. Rec., pages 80-83, Anaheim, California, 2-9 Nov. 1996.

[73] T. Takahashi, S. Konno, T. Hamada, M. Miyajima, S. Kubota, A. Nakamoto, A. Hitachi, E. Shibamura, and T. Doke. Average energy expended per ion pair in liquid xenon. Phys. Rev. A., 12(5):1771-1775, 1975. doi: https: //doi.org/10.1103/PhysRevA.12.1771.

[74] G. Jaffé. Zur theorie der ionisation in kolonnen. Ann. Phys., 347(12):303-344, 1913. doi: https://doi.org/10.1002/andp.19133471205.

[75] H. Kramers. On a modification of Jaffé's theory of column-ionization. Physica, 18(10):665-675, 1952. doi: 10.1016/S0031-8914(52)80255-1.

[76] D. Yu. Akimov et al. Two-phase xenon emission detector with electron multiplier and optical readout by multipixel avalanche geiger photodiodes. JINST, 8:P05017, 2013. doi: https://doi.org/10.1088/1748-0221/8/05/P05017. 
[77] G.F. Knoll. Radiation Detection and Measurement. John Wiley and Sons, Inc., Hoboken, New Jersey, 4th edition, 2010.

[78] Eric. W. Weisstein. Newton Coates Formulas. From MathWorldA Wolfram Web Resource. URL http://mathworld.wolfram.com/ Newton-CotesFormulas.html. Last accessed on 2019-11-14.

[79] Glen Cowan. Statistical Data Analysis. Oxford University Press Inc., Oxford, United Kingdom, 1998. 